\title{
Right-handed charged currents in the era of the Large Hadron Collider
}

\author{
S. Alioli, ${ }^{a}$ V. Cirigliano, ${ }^{b}$ W. Dekens, ${ }^{b, c}$ J. de Vries $^{d}$ and E. Mereghetti ${ }^{b}$ \\ ${ }^{a}$ CERN Theory Division, \\ CH-1211, Geneva 23, Switzerland \\ ${ }^{b}$ Theoretical Division, Los Alamos National Laboratory, \\ Los Alamos, NM 87545, U.S.A. \\ ${ }^{c}$ New Mexico Consortium, Los Alamos Research Park, \\ Los Alamos, NM 87544, U.S.A. \\ ${ }^{d}$ Nikhef, Theory Group, \\ Science Park 105, 1098 XG, Amsterdam, The Netherlands \\ E-mail: simone.alioli@cern.ch, cirigliano@lanl.gov, \\ wdekens@newmexicoconsortium.org, jordy.de.vries@nikhef.nl, \\ emereghetti@lanl.gov
}

ABStract: We discuss the phenomenology of right-handed charged currents in the framework of the Standard Model Effective Field Theory, in which they arise due to a single gauge-invariant dimension-six operator. We study the manifestations of the nine complex couplings of the $W$ to right-handed quarks in collider physics, flavor physics, and low-energy precision measurements. We first obtain constraints on the couplings under the assumption that the right-handed operator is the dominant correction to the Standard Model at observable energies. We subsequently study the impact of degeneracies with other Beyondthe-Standard-Model effective interactions and identify observables, both at colliders and low-energy experiments, that would uniquely point to right-handed charged currents.

Keywords: Beyond Standard Model, CP violation, Higgs Physics

ArXiv ePrint: 1703.04751 


\section{Contents}

1 Introduction 1

2 Right-handed charged currents at colliders 3

$2.1 W$ production 4

2.2 Associated production of a Higgs and a $W^{ \pm}$boson 6

2.3 Vector boson fusion $\quad 9$

2.4 Single-top production and $W$-boson helicity fractions in top decays 11

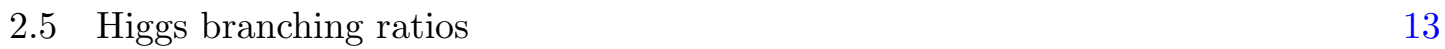

$\begin{array}{lll}2.6 & \text { Summary of collider bounds } & 15\end{array}$

3 Low-energy effective Lagrangian $\quad \mathbf{1 5}$

$\begin{array}{lll}3.1 & \text { Tree-level effective Lagrangian } & 16\end{array}$

3.2 One-loop contributions to $\Delta F=0$ and $\Delta F=1$ operators $\quad 17$

4 Leptonic and semileptonic charged-current decays $\quad 19$

$5 \Delta F=0$ processes: electric dipole moments 24

$6 \quad \Delta S=1$ processes $\quad 26$

$\begin{array}{ll}6.1 \epsilon^{\prime} / \epsilon & 26\end{array}$

$6.2 K_{L} \rightarrow \pi^{0} e^{+} e^{-} \quad 28$

$7 \Delta B=1$ and $\Delta B=2$ processes $\quad 29$

7.1 The $B \rightarrow X_{d, s} \gamma$ branching ratio $\quad 30$

7.2 The $B \rightarrow X_{d, s} \gamma$ CP asymmetry $\quad 30$

7.3 The $B \rightarrow K^{* 0} \gamma$ CP asymmetry 31

$\begin{array}{lll}7.4 & B_{q}-\bar{B}_{q} \text { mixing } & 31\end{array}$

8 Single-coupling constraints $\quad 32$

$\begin{array}{lll}8.1 \text { Summary } & 36\end{array}$

9 Identifying right-handed currents at low and high energy 39

$\begin{array}{lll}9.1 & \text { Low-energy probes } & 39\end{array}$

$\begin{array}{lll}9.2 & \text { Collider probes } & 41\end{array}$

9.3 Anomalous $W t b$ couplings 46

$\begin{array}{ll}10 \text { Conclusion } & 49\end{array}$

A Additional input for CKM fits $\quad \mathbf{5 1}$

A.1 $B \rightarrow J / \psi K \quad 52$

A.2 $\Delta F=1$ neutral current decays $\quad 53$

A.3 $\Delta F=2$ processes $\quad 55$ 
$\begin{array}{lll}\text { A.3.1 } & B-\bar{B} \text { oscillations } & 56\end{array}$

$\begin{array}{lll}\text { A.3.2 } & \varepsilon_{K} & 57\end{array}$

B Two-loop contributions to the electron EDM 58

\section{Introduction}

The existence of right-handed charged currents (RHCC) is a distinctive signature of leftright symmetric extensions of the Standard Model (SM) [1-3]. This class of models is quite attractive as it allows parity to be restored at high energies by extending the SM gauge symmetries to $\mathrm{SU}(3)_{c} \times \mathrm{SU}(2)_{R} \times \mathrm{SU}(2)_{L} \times \mathrm{U}(1)_{B-L}$. An explanation for parity violation is then provided by the spontaneous symmetry breaking of this extended gauge group. Moreover, $\mathrm{TeV}$ scale left-right theories provide an appealing realization of the seesaw mechanism for neutrino masses [4]. In light of the ongoing efforts to search for new physics at the Large Hadron Collider (LHC) and in low-energy precision measurements, it is timely to assess the status and prospects of detecting signals of right-handed charged currents over a broad spectrum of probes.

In this paper, we consider a setup in which RHCC interactions manifest themselves at observable energies, including the scales probed at colliders, through a single $\mathrm{SU}(3)_{c} \times$ $\mathrm{SU}(2)_{L} \times \mathrm{U}(1)_{Y}$-invariant dimension-six operator [5, 6], namely

$$
\mathcal{L}_{6, q q \varphi \varphi}=\frac{2}{v^{2}} i \tilde{\varphi}^{\dagger} D_{\mu} \varphi \bar{u}_{R}^{i} \gamma^{\mu} \xi_{i j} d_{R}^{j}+\text { h.c. }
$$

where $D_{\mu}$ is the covariant derivative, $\varphi$ is the Higgs doublet, $\tilde{\varphi}=i \sigma_{2} \varphi^{*}, v=246 \mathrm{GeV}$ is the Higgs vacuum expectation value, $i$ and $j$ are generation indices, and we work in the quark mass eigenbasis. After electroweak symmetry breaking this operator gives rise to a coupling of the $W^{ \pm}$boson to a right-handed charged current. In the unitary gauge we have

$$
\mathcal{L}_{6, q q \varphi \varphi}=\frac{g}{\sqrt{2}}\left[\xi_{i j} \bar{u}_{R}^{i} \gamma^{\mu} d_{R}^{j} W_{\mu}^{+}\right]\left(1+\frac{h}{v}\right)^{2}+\text { h.c. },
$$

where $g$ is the $\mathrm{SU}(2)_{L}$ gauge coupling. The operator in eq. (1.1) arises in left-right symmetric models from the mixing between the charged gauge bosons of the $\mathrm{SU}(2)_{R}$ and $\mathrm{SU}(2)_{L}$ gauge groups. In this case $\xi_{i j}$ is proportional to a unitary $3 \times 3$ matrix, the right-handed analog of the Cabibbo-Kobayashi-Maskawa (CKM) matrix. Here we do not commit to a specific model, so that $\xi$ is a generic $3 \times 3$ matrix, with 9 independent complex parameters. The elements $\xi_{i j}$ scale as $\xi_{i j} \sim \mathcal{O}\left(v^{2} / \Lambda^{2}\right)$, where $\Lambda$ is the scale of new physics. We work within the framework of the SM effective field theory (SMEFT), in which it is a valid approximation to only consider dimension-six operators as long as there is a gap between the scale of new physics and the largest energy scale in the problem. For low-energy observables the largest energy scale will be the electroweak scale, such that we have the requirement 
$\Lambda>v$. Instead, due to the larger energies available at colliders, the effects of eq. (1.1) can be investigated in $p p$ collisions at the LHC if one assumes that $\Lambda>$ few $\mathrm{TeV}$.

Although we do not restrict ourselves to a specific model it is worthwhile to mention how eq. (1.2) can be induced in UV-complete models. For example, in the minimal leftright symmetric model (see e.g. ref. [7]) the effective operator arises due to the mixing between left- and right-handed $W$ bosons. In this set-up, after integrating out the heavy right-handed $W$ boson, we can identify $\xi_{i j} \sim\left(\kappa \kappa^{\prime} / v_{R}^{2}\right)\left(V_{R}\right)_{i j} e^{i \alpha}$ where $\kappa$ and $\kappa^{\prime}$ are vacuum expectation values of the order of the electroweak scale, $v_{R} \gg v$ is the right-handed scale, $\alpha$ is a CP-violating phase arising from the extended Higgs sector of the model, and $V_{R}$ the right-handed analogue of the CKM matrix. With further assumptions, such as explicit $P$ and/or $C$ symmetry at high energies, $V_{R}$ can even be calculated in terms of SM quantities, such as quark masses and CKM elements, and the new model parameters $\kappa, \kappa^{\prime}$, and $\alpha$ [810]. In this way, our results can be used in phenomenological analyses of UV-complete scenarios, although care must be taken as other effective operators might be induced at the matching scale.

The operator (1.1) has several interesting manifestations, both at high- and low-energy. At colliders, it affects the production and polarization of $W$ bosons. Furthermore, the operator (1.1) affects the production cross section of the Higgs boson, both in associated production with a $W$ boson and in the vector boson fusion (VBF) channel. As we will discuss, invariance under the SM gauge group causes eq. (1.2) to modify not only the $W q q^{\prime}$ vertex, but also the $H W q q^{\prime}$ interaction. This latter interaction produces a very different dependence of the Higgs production cross section on kinematic variables such as the partonic center of mass energy or the Higgs transverse momentum. This in turn results in a large enhancement of the $\xi$-mediated cross section compared to the SM, especially for $W H$ associated production. As a consequence, we will see that, for the first two rows of the $\xi$ matrix, processes involving the Higgs are already more constraining than single $W$ production. The third row of the $\xi$ matrix is directly constrained by single-top production, and top decay. In particular, the measurement of the $W$ polarization in top decay allows for a direct access to the Lorentz structure of the $W t b$ vertex, and to test its left-handed nature.

The operator (1.1) leaves a distinctive trace at low energy as well. Indeed, it is the only dimension-six operator in the SMEFT that induces a tree-level charged-current coupling of left-handed leptons to right-handed quarks, thus affecting baryon $\beta$ decays, and meson leptonic and semileptonic charged-current decays. We will see that, under the assumption that the SM is modified predominantly by a RHCC at the high energy scale $\Lambda$, low-energy probes provide very stringent constraints on the first two rows of the $\xi$ matrix. However, the most constraining observables are degenerate enough that, by introducing new physics beyond the $\xi_{i j}$, the bounds can be weakened to levels that are comparable to collider sensitivities. Less degenerate observables, such as decay correlations in the neutron $\beta$ decays, suffer from comparatively large theoretical uncertainties, so that, once again, they probe the $\xi_{i j}$ at levels comparable to collider experiments. One of our main findings is that once one tries to remove degeneracies by identifying observables that are sensitive primarily to $\xi_{i j}$, collider searches and low-energy probes have comparable sensitivity, and it is of great value to pursue both. 
In addition to observables that are at least in principle directly sensitive to RHCC, we also consider indirect bounds, both at high- and low-energy. Some of the most stringent indirect limits arise through top-quark loops which induce large corrections, enhanced by $m_{t} / m_{d_{j}}$, to the bottom-Yukawa coupling and dipole operators. In turn, the bottom Yukawa induces $h \rightarrow b \bar{b}$, while the dipole operators contribute to $B \rightarrow X_{s, d} \gamma$, the rare decay $K_{L} \rightarrow \pi^{0} e^{+} e^{-}$, and hadronic electric dipole moments (EDMs). We will see that the constraints from these loop processes are several orders of magnitude stronger than direct constraints from top production and decay. Furthermore, RHCC of light quarks induce tree-level contributions to EDMs and direct CP-violation (CPV) in kaon decays [11]. The stringent bounds on hadronic EDMs and the experimental value of $\epsilon^{\prime} / \epsilon$ can therefore be used to rule out couplings larger that $\operatorname{Im} \xi_{u d, u s} \sim 10^{-6}-10^{-7}$, suggesting a very high righthanded scale. While these indirect probes are certainly more "degenerate" than direct observables (i.e. they receive contributions from several other dimension-six operators in the SMEFT), the bounds that they imply are nonetheless very significant. Within the SMEFT these limits put stringent bounds on certain directions in the space of dimensionsix Wilson coefficients, thereby imposing non-trivial constraints on new physics scenarios.

This paper is organized as follows. We start by investigating the constraints coming from colliders in section 2. We discuss direct $W$ production in section 2.1, associated production of a Higgs and a $W$ boson in section 2.2, and Higgs production via VBF in section 2.3. We then consider constraints on the $\xi_{t j}$ elements coming from top production and decay in section 2.4, and from the decays of the Higgs in section 2.5. To connect to lowenergy observables, in section 3, we integrate out the heavy SM particles and match onto a low-energy effective Lagrangian. In section 4 we consider the constraints coming from $\beta$ decay, and from leptonic and semileptonic meson decays. We then discuss observables sensitive to non-leptonic operators induced by the operator (1.1), organizing the discussion in $\Delta F=0$ observables (section 5 ), which consists of hadronic EDMs, $\Delta S=1$ observables, including $\epsilon^{\prime} / \epsilon$ and $K_{L} \rightarrow \pi^{0} e^{+} e^{-}$(section 6 ), and $\Delta B=1$ observables, mainly related to inclusive and exclusive $b \rightarrow s, d \gamma$ transitions (section 7). In section 8 , we obtain limits on the real and imaginary part of $\xi_{i j}$ by taking into account all the observables discussed above, in a scenario in which only one of the elements is turned on at the high scale. In section 9 we discuss strategies to unambiguously identify the signal of a RHCC, both at low energies, and in associated production of a Higgs and a $W$. Finally, in section 9.3 we consider more in detail the $W t b$ vertex. We conclude in section 10.

\section{Right-handed charged currents at colliders}

In this section we study the effects of the RHCC operator $\xi$ on several processes of interest at the LHC. We focus on $W$ production, associated production of a $W$ and a Higgs boson, Higgs production via vector boson fusion, and single-top production, as we expect these processes to be the most sensitive to RHCC. For all these processes, we include NLO QCD corrections to both the SM and BSM contributions, and, with the exception of single-top production, we implement the processes in the POWHEG BOX V2 [12-14]. We also consider the effect of the $\xi$ operator on the decays of the top-quark and the Higgs boson. 


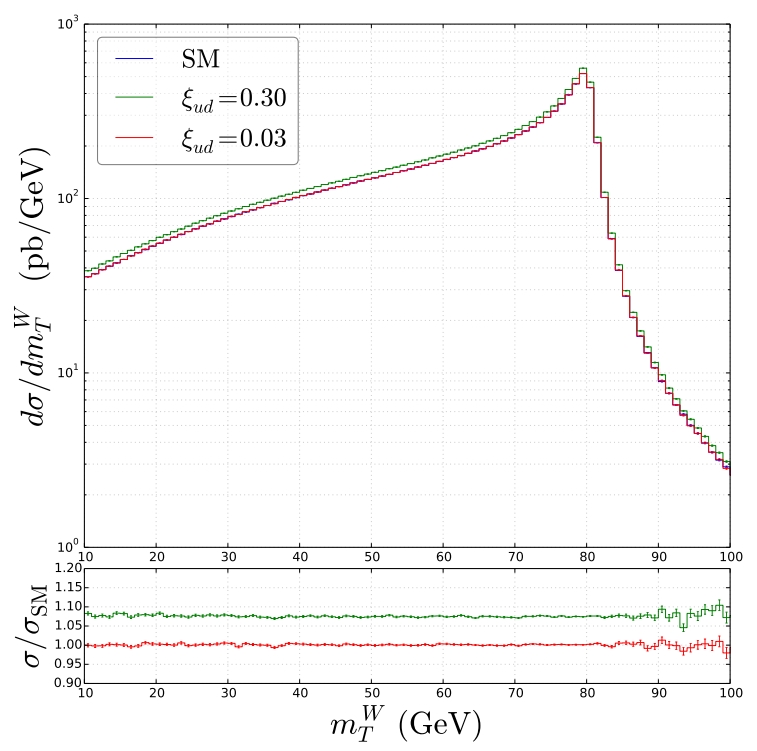

Figure 1. Transverse mass distribution $m_{T}^{W}$ in $p p \rightarrow W^{+} \rightarrow e^{+} \nu$ at $\sqrt{S}=13 \mathrm{TeV}$ in the presence of right-handed charged currents.

Throughout this section we always consider $n_{f}=5$ massless quarks and we include the exact top-quark mass dependence when necessary. For processes involving light quarks only, this is justified by the fact that the interference of the right-handed currents with the $\mathrm{SM}$ is suppressed by two insertions of the light quark masses. The interference is negligible for values of the couplings $\xi_{i j} \gg y_{u_{i}} y_{d_{j}}$, where $y_{u_{i}, d_{j}}$ are the SM Yukawa couplings. Even in the most favorable case, $\xi_{c b}$, neglecting the charm and bottom masses is reasonable for $\xi_{c b}>10^{-3}$, a level that, as we will see, is far from the sensitivity that can be reached by present collider experiments. In the case of single-top production and top decays, interference terms are important for $\xi_{t b} \sim y_{b} y_{t}$. While in this case the corrections are more relevant, they are still subleading with respect to terms quadratic in $\xi_{t b}$ for the values of the coupling accessible at colliders.

\subsection{W production}

The first process we analyze to look for manifestations of RHCC is $W^{ \pm}$production. This process is accurately measured at the LHC, both at the level of the inclusive cross section as well as for differential distributions [15-25]. Precise high-order calculations of the SM background are available up to fixed NNLO QCD corrections [26-28] and also include the resummation of the vector-boson transverse momentum [29, 30]. More recently, the interface of the NNLO predictions with the parton shower has been presented in ref. [31]. A careful quantitative assessment of the size of the corrections at different orders, both in QCD and EW, has been presented in ref. [32]. For this study, we have calculated the NLO QCD corrections to the partonic processes mediated by RHCC and interfaced with the parton shower according to the POWHEG method, extending the original work of ref. [33].

The contribution of the RHCC operator to $W^{ \pm}$-production observables that are symmetric under the exchange of the charged lepton and the neutrino momenta, is identical to 

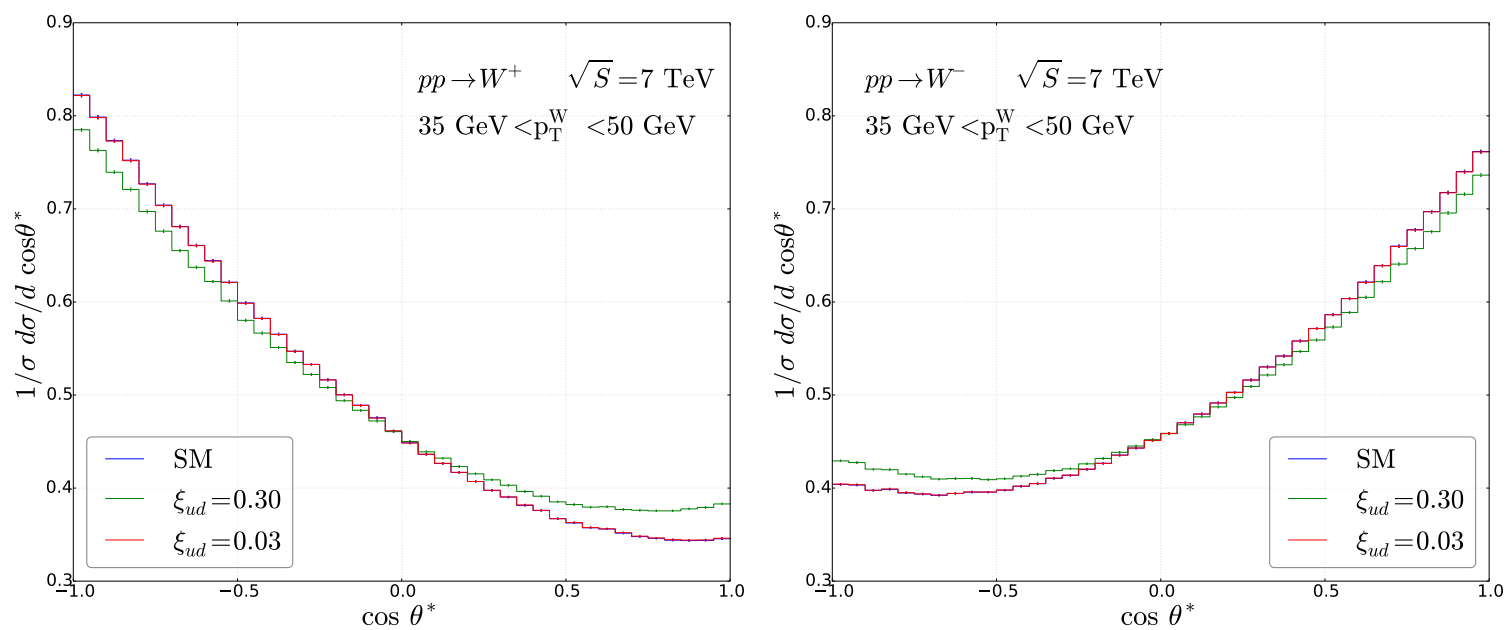

Figure 2. Polar angle $\cos \theta^{*}$ distribution in $p p \rightarrow W^{+} \rightarrow e^{+} \nu$ and $p p \rightarrow W^{-} \rightarrow e^{-} \bar{\nu}$ at $\sqrt{S}=$ $7 \mathrm{TeV}$, in the $\mathrm{SM}$ and in the presence of $\xi_{u d} . \theta^{*}$ is the polar angle of the charged lepton, measured in the $W$ boson rest frame, with the $z$-axis chosen to be oriented along the $W$ direction in the lab frame.

the SM contribution after the replacement of the CKM elements $V_{i j}$ by $\xi_{i j}$. For example, in figure 1 we show the differential distribution with respect to the $W$ transverse mass which is defined as [34]

$$
m_{T}^{W}=\sqrt{2\left|p_{T l}\right|\left|p_{T \nu}\right|\left(1-\cos \Delta \phi_{l \nu}\right)} .
$$

Here $p_{T l}$ and $p_{T \nu}$ are the charged-lepton and the neutrino transverse momenta, respectively, and $\Delta \phi_{l \nu}$ is their azimuthal separation. We evaluate the cross section at $\sqrt{S}=$ $13 \mathrm{TeV}$ for the SM (blue curve), $\xi_{u d}=0.3$ (green curve), and $\xi_{u d}=0.03$ (red curve). The effect of the RHCC amounts to a rescaling of the cross section. Since the correction is quadratic in $\xi_{i j}$, choosing $\xi_{u d}=0.3$ gives approximately a $10 \%$ correction to the SM prediction, while $\xi_{u d}=0.03$ gives sub-permille corrections. Presently, the $W^{ \pm}$cross section at $13 \mathrm{TeV}$ is known with roughly $3 \%$ experimental uncertainty and a similar theoretical uncertainty [16], implying that the bound on $\xi_{u d}$ that can be extracted from the $W^{ \pm}$cross section is at best around $\left|\xi_{u d}\right| \lesssim 0.2$. Due to PDF suppression, the bounds on the other elements of the $\xi_{i j}$ matrix are even weaker. As we discuss in section 2.2, with current sensitivity, the associated production of a Higgs and a $W$ boson is already more constraining than $W$ production. For this reason, we do not list the bounds coming from $W$ production on the various $\xi_{i j}$ elements.

The angular distribution of the charged leptons in $W^{ \pm}$decay is sensitive to the lefthanded nature of the $W$ boson in the SM [35]. One might therefore expect that angular distributions can provide stronger constraints. In figure 2 we plot the differential distribution with respect to $\cos \theta^{*}$, where $\theta^{*}$ is the polar angle of the charged lepton in the $W$-boson rest frame, with the $z$-axis chosen to be in the direction of the $W$-boson momentum in the laboratory frame. The differential $\cos \theta^{*}$ distribution is related to the $W$ boson polarization 

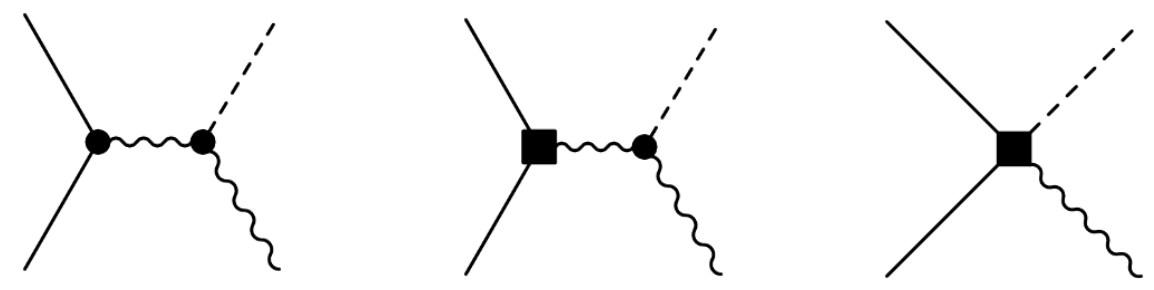

Figure 3. Leading-order contribution to the associated production of a Higgs and a vector boson. Dots denote SM vertices, while squares denote an insertion of $\xi$.

fractions, $F_{0}, F_{L}$, and $F_{R}$ by the relations [35]

$$
\begin{aligned}
F_{0} & =\int d \cos \theta^{*}\left(2-5 \cos ^{2} \theta^{*}\right) \frac{1}{\sigma} \frac{d \sigma}{d \cos \theta^{*}}, \\
F_{L} & =\int d \cos \theta^{*}\left(-\frac{1}{2} \mp \cos \theta^{*}+\frac{5}{2} \cos ^{2} \theta^{*}\right) \frac{1}{\sigma} \frac{d \sigma}{d \cos \theta^{*}}, \\
F_{R} & =\int d \cos \theta^{*}\left(-\frac{1}{2} \pm \cos \theta^{*}+\frac{5}{2} \cos ^{2} \theta^{*}\right) \frac{1}{\sigma} \frac{d \sigma}{d \cos \theta^{*}},
\end{aligned}
$$

where the upper (lower) sign is for $W^{+(-)}$.

Figure 2 depicts the $\cos \theta^{*}$ distribution for the SM (blue curve), for $\xi_{u d}=0.3$, and for $\xi_{u d}=0.03$. These predictions were evaluated at $\sqrt{S}=7 \mathrm{TeV}$ and we applied the same $p_{T}^{W}$ cuts as used in the analysis of ref. [18]. We observe that $\xi_{u d}$ does not affect the longitudinal fraction $F_{0}$, while it increases the fraction of right-handed polarized $W$ 's, and decreases $F_{L}-F_{R}$. We find the helicity fractions to be given by

$$
F_{0}=0.21, \quad F_{L}=0.54 \frac{1+0.3 \xi_{u d}^{2}}{1+0.8 \xi_{u d}^{2}} \quad F_{R}=0.25 \frac{1+1.9 \xi_{u d}^{2}}{1+0.8 \xi_{u d}^{2}},
$$

for $35 \mathrm{GeV}<p_{T}^{W}<50 \mathrm{GeV}$, and

$$
F_{0}=0.19, \quad F_{L}=0.55 \frac{1+0.4\left|\xi_{u d}\right|^{2}}{1+0.8\left|\xi_{u d}\right|^{2}} \quad F_{R}=0.26 \frac{1+1.8\left|\xi_{u d}\right|^{2}}{1+0.8\left|\xi_{u d}\right|^{2}}
$$

for $p_{T}^{W}>50 \mathrm{GeV}$. These helicity fractions were measured in ref. [18] with uncertainties of roughly $20 \%$ on $F_{L}-F_{R}$ and larger uncertainties on $F_{0}$. The significant uncertainties allow for rather large values of $\xi_{u d}$, up to $\xi_{u d} \sim 0.4$. The contributions to the $W$ helicity fractions involving other elements of the $\xi_{i j}$ matrix are further suppressed by the respective PDF's resulting in even weaker bounds. We conclude that at the moment the $W$ polarization fractions do not provide strong constraints on the effects of RHCC.

\subsection{Associated production of a Higgs and a $W^{ \pm}$boson}

As for single W-boson production, $W H$ associated production is available at NNLO in QCD [36-38] and is matched to the parton shower up to the NNLO+PS level [39].

A representative set of tree-level diagrams contributing to associated $W H$ production at LO in presence of RHCC is shown in figure 3. The first diagram denotes the SM 


\begin{tabular}{|c|c|c|c|c|c|c|c|}
\hline & & $\left|\xi_{u d}\right|^{2}$ & $\left|\xi_{u s}\right|^{2}$ & $\left|\xi_{u b}\right|^{2}$ & $\left|\xi_{c d}\right|^{2}$ & $\left|\xi_{c s}\right|^{2}$ & $\left|\xi_{c b}\right|^{2}$ \\
\hline \multirow{3}{*}{$\sigma_{W^{+} H}(\mathrm{pb})$} & central & 230 & 158 & 66.4 & 12.7 & 7.48 & 2.84 \\
\hline & scale & \pm 4 & \pm 3 & $\begin{array}{l}+0.8 \\
{ }_{-0.4}\end{array}$ & $\begin{array}{l}+0.2 \\
{ }_{-0.1}^{0.2}\end{array}$ & $\begin{array}{l}+0.08 \\
-0.04\end{array}$ & $\begin{array}{l}+0.08 \\
{ }_{-0.04}\end{array}$ \\
\hline & PDF & 20 & 36.0 & 3.2 & 1.8 & 2.32 & 0.48 \\
\hline \multirow{3}{*}{$\sigma_{W^{-} H}(\mathrm{pb})$} & central & 100 & 17.4 & 6.64 & 50.4 & 7.72 & 2.84 \\
\hline & scale & \pm 2 & $\begin{array}{l}+2.8 \\
-2.4\end{array}$ & $\begin{array}{l}+0.08 \\
{ }_{-0.04}\end{array}$ & $\begin{array}{l}+0.8 \\
{ }_{-0.4}\end{array}$ & $\begin{array}{l}{ }_{-0.04}^{+0.08} \\
\end{array}$ & $\begin{array}{l}+0.08 \\
{ }_{-0.04}\end{array}$ \\
\hline & PDF & 10.4 & 4.00 & 1.12 & 3.2 & 2.08 & 0.48 \\
\hline
\end{tabular}

Table 1. Corrections to the $W^{ \pm} H$ cross section induced by the RHCC $\xi_{i j}$ at $\sqrt{S}=8 \mathrm{TeV}$. The total cross section is $\sigma_{W^{ \pm} H}=\sigma_{W^{ \pm} H}^{S M}+\sum a_{i j}\left|\xi_{i j}\right|^{2}$, where the sum is over all light quarks. The values and theoretical uncertainties of $a_{i j}$ are given in the table.

contribution. The remaining two diagrams involve an insertion of $\xi$, which is denoted by a square. In addition to a contribution similar to the SM (the second diagram), the $\xi$ operator induces a local $\left(\bar{q}^{\prime} q\right)_{R} h W$ interaction (the third diagram), which leads to a large enhancement of the RHCC contribution. The interference of the right-handed currents with the SM is suppressed by two insertions of the light quark masses and is negligible for values of the couplings that can be probed at the LHC. We therefore focus on the contributions quadratic in $\xi$.

We computed the NLO QCD corrections to the RHCC contributions to the $W H$ cross section, also considering the decay of the $W$ into leptons. This implementation builds upon the original NLO+PS POWHEG BOX code in [40]. The SM NLO cross section at $\sqrt{S}=8 \mathrm{TeV}$ is

$$
\sigma_{W^{+} H}=(0.461 \pm 0.021) \mathrm{pb}, \quad \sigma_{W^{-} H}=(0.264 \pm 0.017) \mathrm{pb},
$$

where we used the NLO PDF sets CT10, MSTW08, and NNPDF2.3. The error is dominated by PDF uncertainties while scale variations are about $(2-3) \%$. In table 1 we give the cross sections induced by $\xi$ at $\sqrt{S}=8 \mathrm{TeV}$. The central value is evaluated at the scale $\mu=m_{H}+m_{W}$. As for the SM results, the errors are dominated by PDF variations that are roughly $10 \%$. As expected, PDF errors are larger for processes involving the strange PDF, which is not known at the same level as the PDFs of the lighter quarks. The cross sections at 13 and $14 \mathrm{TeV}$ have similar theoretical uncertainties.

Using the total cross sections in table 1 , we construct the production signal strength in the presence of the RHCC,

$$
\mu_{W H}^{\xi}=1+\frac{\sigma_{W^{+} H}^{\xi}+\sigma_{W^{-} H}^{\xi}}{\sigma_{W^{+} H}^{S M}+\sigma_{W^{-} H}^{S M}} .
$$

Our results for the production signal strengths at $\sqrt{S}=8,13$ and $14 \mathrm{TeV}$ are summarized in the upper half of table 2. As discussed in section 2.5, the first two rows of the $\xi_{i j}$ matrix do not significantly affect the Higgs branching ratios. We can thus safely assume that Higgs 


\begin{tabular}{|c|c|cccccc|}
\hline & $\sqrt{S}$ & $\left|\xi_{u d}\right|^{2}$ & $\left|\xi_{u s}\right|^{2}$ & $\left|\xi_{u b}\right|^{2}$ & $\left|\xi_{c d}\right|^{2}$ & $\left|\xi_{c s}\right|^{2}$ & $\left|\xi_{c b}\right|^{2}$ \\
\hline & $8 \mathrm{TeV}$ & $451 \pm 29$ & $250 \pm 57$ & $101 \pm 7$ & $87 \pm 7$ & $22 \pm 6$ & $8 \pm 2$ \\
$\mu_{W H}-1$ & $13 \mathrm{TeV}$ & $663 \pm 42$ & $381 \pm 80$ & $164 \pm 11$ & $142 \pm 10$ & $42 \pm 10$ & $16 \pm 3$ \\
& $14 \mathrm{TeV}$ & $703 \pm 45$ & $406 \pm 84$ & $177 \pm 12$ & $153 \pm 11$ & $46 \pm 11$ & $18 \pm 3$ \\
\hline & & $\left|\xi_{u d}\right|$ & $\left|\xi_{u s}\right|$ & $\left|\xi_{u b}\right|$ & $\left|\xi_{c d}\right|$ & $\left|\xi_{c s}\right|$ & $\left|\xi_{c b}\right|$ \\
\hline ATLAS, CMS [41] & $8 \mathrm{TeV}$ & 0.04 & 0.05 & 0.08 & 0.08 & 0.19 & 0.30 \\
ATLAS [44] & $13 \mathrm{TeV}$ & 0.04 & 0.06 & 0.08 & 0.09 & 0.18 & 0.28 \\
future & $14 \mathrm{TeV}$ & 0.02 & 0.03 & 0.04 & 0.05 & 0.09 & 0.13 \\
\hline
\end{tabular}

Table 2. Signal strength for the $W H$ production channel at $\sqrt{S}=8 \mathrm{TeV}, 13 \mathrm{TeV}$, and $14 \mathrm{TeV}$, and $90 \%$ CL bounds on $\xi_{i j}$. The projected bounds at $14 \mathrm{TeV}$ assume $20 \%$ uncertainties on $\mu_{W H}$.

decays are SM-like and we simply use the production signal strength in the $W H$ channel in our analysis. The combined result from the ATLAS and CMS collaborations is [41]

$$
\mu_{W H}(8 \mathrm{TeV})=0.89_{-0.38}^{+0.40} .
$$

In the lower half of table 2 we list the $90 \%$ CL bounds on $\left|\xi_{i j}\right|$, with the assumption that the SM is modified only by a RHCC at the high-energy scale $\Lambda \gg v .^{1}$ The constraints are much stronger than those extracted from $W^{ \pm}$production. The ATLAS collaboration also published preliminary results obtained at $13 \mathrm{TeV}$ [44]

$$
\mu_{W H}(13 \mathrm{TeV})=0.33_{-0.92}^{+0.95} .
$$

The larger contribution of the RHCC operators to the cross section is compensated by the larger experimental errors, resulting in similar constraints as obtained from the $8 \mathrm{TeV}$ data.

In table 2 we also show the signal strength and projected bounds at $\sqrt{S}=14 \mathrm{TeV}$. The RHCC contributions to the cross section grow faster than the SM contributions, resulting in a greater sensitivity at the LHC Run 2. The projected bounds in table 2 assume a $20 \%$ uncertainty on the $\mu_{W H}$ signal strength. For all coefficients the improvement is roughly a factor of 2 .

The pattern of the constraints in table 2 can be simply understood in terms of the parton distributions. $W H$ production is most sensitive to $\xi_{u d}$, which involves two valence quarks in the proton, followed by the couplings with one valence quark, $\xi_{u s}, \xi_{u b}$, and $\xi_{c d}$. The bounds become weaker for $\xi_{c s}$ and $\xi_{c b}$ as the PDFs involve two sea quarks in these cases. While the current and projected bounds on $\xi_{u d}, \xi_{u s}, \xi_{c d}$, and $\xi_{c s}$ are at least a factor of 10 smaller than the corresponding CKM matrix element, the $W H$ cross section allows for values of $\xi_{u b}$ and $\xi_{c b}$ that are much larger than $V_{u b}$ and $V_{c b}$. As we will discuss, this possibility is excluded by inclusive and exclusive $B$ decays.

\footnotetext{
${ }^{1}$ Since the coefficients $\xi_{i j}$ depend very mildly on the initial scale $\Lambda$ [42, 43], we do not specify its precise value.
} 


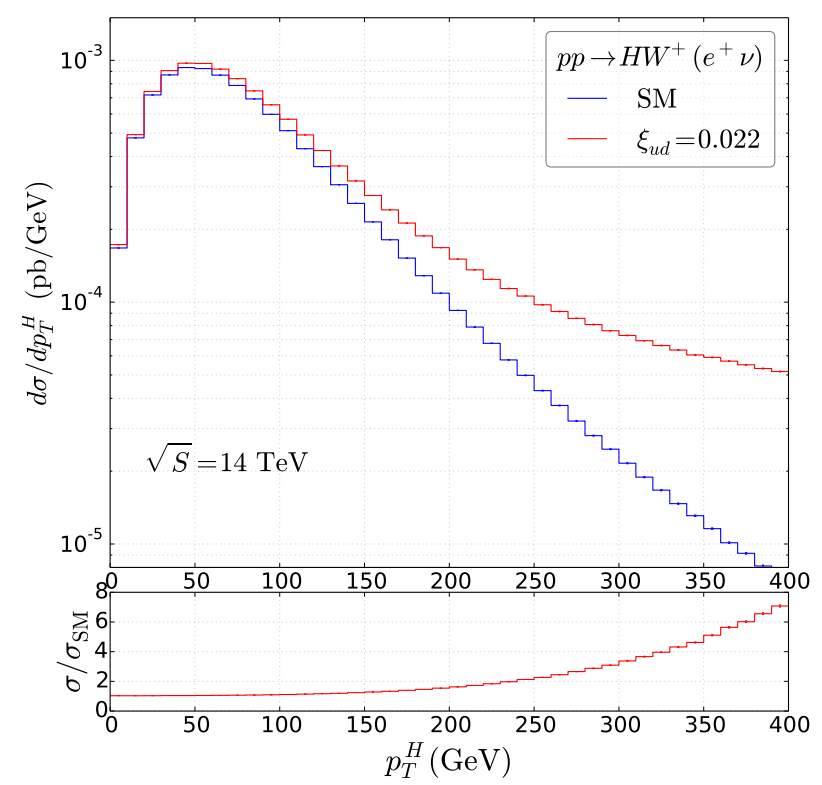

Figure 4. Cross section for the production of $H W^{+}$, with $W^{+} \rightarrow \nu e^{+}$, at $\sqrt{S}=14 \mathrm{TeV}$, differential with respect to the Higgs $p_{T}$.

We have focused so far on the signal strengths alone. Differential distributions can provide additional valuable information. In particular, as shown in figure 4, the RHCC contribution is enhanced for large values of the Higgs transverse momentum, or of the $W H$ invariant mass, so that the study of differential distributions at the current and future LHC runs could further improve the collider bounds on $\xi$. In section 9 we will explore in more detail how to construct observables that can discriminate between RHCC and corrections to the $W H$ cross section from other dimension-six operators in the SMEFT.

\subsection{Vector boson fusion}

The production rate for an Higgs boson via vector boson fusion (VBF) is also sensitive to the RHCC. The tree-level diagrams contributing to VBF Higgs production are shown in figure 5. As for $W H$ associated production, one topology is identical to the SM with the CKM matrix replaced by $\xi_{i j}$. In addition, the $\xi$ operator induces a $\bar{q}^{\prime} q h W$ vertex depicted in the last diagram of figure 5. This topology again leads to an enhancement with respect to the SM, albeit not as numerically significant as for $W H$ associated production.

The total cross section for Higgs production through VBF has been recently computed in the SM at $\mathrm{N}^{3} \mathrm{LO}$ in QCD [45]. Fully-differential distributions are available up to NNLO [46] and the interface with parton showering is available at NLO+PS accuracy [47]. For this study, we computed the NLO QCD corrections to both the SM and the RHCC VBF cross sections, building upon the POWHEG implementation presented in ref. [47]. We find that the total VBF cross section is

$$
\frac{\sigma_{\mathrm{VBF}}(8 \mathrm{TeV})}{\mathrm{pb}}=1.6\left(1+16.4\left|\xi_{u d}\right|^{2}+9.1\left|\xi_{u s}\right|^{2}+8.5\left|\xi_{u b}\right|^{2}+6.2\left|\xi_{c d}\right|^{2}+2.6\left|\xi_{c s}\right|^{2}+1.1\left|\xi_{c b}\right|^{2}\right),
$$



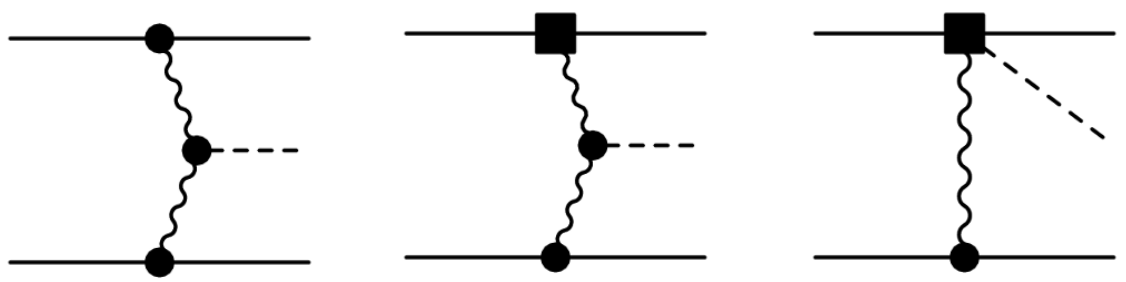

Figure 5. Leading order contribution to Higgs production via vector boson fusion. Dots denote SM vertices, while squares denotes an insertion of $\xi$.

where we used $\mu=m_{W}$ as the renormalization and factorization scale, and we computed the cross section with the CT10nlo PDF set. PDF and scale variations indicate that the theory uncertainties are below the $10 \%$ level.

In order to disentangle VBF from gluon-fusion contributions, the final state is required to have at least two well-separated jets. The invariant mass of the two jets has to be $m_{j j}>600 \mathrm{GeV}$, while the rapidity separation has to be $\left|y_{j_{1}}-y_{j_{2}}\right|>4.2$. Furthermore, all jets are required to have $p_{T j}>20 \mathrm{GeV}$ and $\left|y_{j}\right|<5$. Within these cuts, the NLO cross section becomes

$$
\frac{\sigma_{\mathrm{VBF}}(8 \mathrm{TeV})}{\mathrm{pb}}=0.30\left(1+12.0\left|\xi_{u d}\right|^{2}+7.3\left|\xi_{u s}\right|^{2}+6.7\left|\xi_{u b}\right|^{2}+5.7\left|\xi_{c d}\right|^{2}+1.3\left|\xi_{c s}\right|^{2}+0.3\left|\xi_{c b}\right|^{2}\right) .
$$

From eqs. (2.11) and (2.12) we see that the VBF channel is sensitive to RHCC, but the modifications to the signal strength are much smaller than for $W H$ associated production. The combined ATLAS and CMS signal strength from Run I is [41]

$$
\mu_{V B F}=1.18 \pm 0.25
$$

This implies that from the signal strength alone we get constraints on, for example, $\left|\xi_{u d}\right|<$ $0.2,\left|\xi_{u b}\right|<0.3$, and $\left|\xi_{c b}\right| \sim 1$. These bounds are considerably weaker than those given in table 2 arising from associated production.

At $14 \mathrm{TeV}$, the cross section within the aforementioned VBF cuts is

$$
\frac{\sigma_{\mathrm{VBF}}(14 \mathrm{TeV})}{\mathrm{pb}}=1.1\left(1+23.2\left|\xi_{u d}\right|^{2}+12.4\left|\xi_{u s}\right|^{2}+11.8\left|\xi_{u b}\right|^{2}+8.7\left|\xi_{c d}\right|^{2}+2.4\left|\xi_{c s}\right|^{2}+1.0\left|\xi_{c b}\right|^{2}\right),
$$

which again signals a smaller sensitivity to $\xi_{i j}$ compared to $W H$ associated production.

One advantage of using VBF is the presence of jets in the final state, which gives an additional handle on the flavor structure of the RHCC. For example, by requiring that at least one of the jets in the final state is $b$ tagged, we can enhance the contributions of the couplings $\xi_{u b}$ and $\xi_{c b}$ at the price of a reduced cross section,

$$
\left.\frac{\sigma_{\mathrm{VBF}}(14 \mathrm{TeV})}{\mathrm{pb}}\right|_{\mathrm{btag}}=0.05\left(1+235\left|\xi_{u b}\right|^{2}+19.3\left|\xi_{c b}\right|^{2}\right) \text {. }
$$




\begin{tabular}{|c|c|c|c|cc|}
\hline$\sqrt{S}(\mathrm{TeV})$ & $\sigma_{t}(\mathrm{pb})$ & $\sigma_{\bar{t}}(\mathrm{pb})$ & $\sigma_{t+\bar{t}}(\mathrm{pb})$ & \multicolumn{2}{|c|}{ Experiment } \\
\hline 7 & $46 \pm 6$ & $23 \pm 3$ & $68 \pm 8$ & ATLAS & {$[48]$} \\
& - & - & $67 \pm 7$ & CMS & {$[49]$} \\
\hline 8 & $57 \pm 4$ & $33 \pm 3$ & $90 \pm 5$ & ATLAS & {$[50]$} \\
& $54 \pm 5$ & $28 \pm 4$ & $84 \pm 8$ & CMS & {$[51]$} \\
\hline \multirow{2}{*}{13} & $156 \pm 28$ & $91 \pm 18$ & $247 \pm 46$ & ATLAS & {$[52]$} \\
& $150 \pm 22$ & $82 \pm 16$ & $232 \pm 31$ & CMS & {$[53]$} \\
\hline
\end{tabular}

Table 3. $t$-channel single-top total cross sections measured by ATLAS and CMS.

After $b$ tagging, the enhancement of the $\xi_{u b}$ and $\xi_{c b}$ contributions to VBF is slightly better than the one quoted in table 2 for $W H$. Since the flavor tag allows for more direct access to specific elements of the $\xi$ matrix, it can be interesting to pursue such observables at the Run II.

\subsection{Single-top production and $W$-boson helicity fractions in top decays}

The processes considered so far ( $W$ production, $W H$ production, and Higgs production via $\mathrm{VBF}$ ) do not constrain the $\xi_{t j}$ elements of the right-handed current matrix. In this section, we consider single-top production, which is sensitive to Wtq couplings, and the $W$-boson helicity fractions in top decay, which probe the $W t b$ coupling, and are sensitive to its left-handed nature.

The largest SM contribution to single-top production at the LHC is via the $t$-channel exchange of a $W$ boson. Smaller contributions arise from the associated production of a top and a $W$ boson and by $s$-channel $W$ exchange. ATLAS published the measurement of the inclusive and differential cross sections at $\sqrt{S}=7,8$, and $13 \mathrm{TeV}$, with luminosities of, respectively, $5 \mathrm{fb}^{-1}, 20.2 \mathrm{fb}^{-1}$ and $3.2 \mathrm{fb}^{-1}[48,50,52]$. CMS published results at $\sqrt{S}=7$, 8 , and $13 \mathrm{TeV}$, with luminosities of $1.56,20$, and $2.3 \mathrm{fb}^{-1}$, respectively $[49,51,53]$. The observed cross sections are summarized in table 3 . The associated production of a top and a $W$ boson, and $s$-channel single-top production have also been observed [54-56]. In our analysis, however, we include only $t$-channel production as this gives the most stringent constraints.

The production of a top-quark in the $t$-channel can be described both in the 5 -flavor scheme, in which the $b$ quark is considered massless and appears in the initial state, and in the 4-flavor scheme, which keeps into account $m_{b}$ effects. The total and differential SM cross sections are known at NNLO in QCD [57] only in the 5-flavor scheme. Top-quark decay is also available at NNLO $[58,59]$. A combination of production and decay in the top-quark narrow-width approximation has been presented in ref. [60]. The calculation in the 4-flavor scheme has been presented at NLO in QCD in ref. [61] and the interface to the parton shower is discussed in ref. [62]. More recently, this process was interfaced with the parton shower at the NLO+PS level, including off-shell and interference effects [63-65]. 


\begin{tabular}{|c|c|c|c|c|c|}
\hline$\sqrt{S}(\mathrm{TeV})$ & & $\mathrm{SM}$ & $\left|\xi_{t d}\right|^{2}$ & $\left|\xi_{t s}\right|^{2}$ & $\left|\xi_{t b}\right|^{2}$ \\
\hline 7 & $\sigma_{t}(\mathrm{pb})$ & $41.9_{-0.9}^{+1.8}$ & $309 \pm 3$ & $85.6 \pm 6.4$ & $36.0 \pm 1.2$ \\
& $\sigma_{\bar{t}}(\mathrm{pb})$ & $22.7_{-1.0}^{+0.9}$ & $89.2 \pm 2.4$ & $58.0 \pm 4.8$ & $24.0 \pm 0.8$ \\
\hline 8 & $\sigma_{t}(\mathrm{pb})$ & $56.4_{-1.1}^{+2.4}$ & $375 \pm 4$ & $110 \pm 8$ & $48 \pm 1.6$ \\
& $\sigma_{\bar{t}}(\mathrm{pb})$ & $30.7_{-1.3}^{+1.1}$ & $115 \pm 3$ & $76 \pm 6.4$ & $32 \pm 1.0$ \\
\hline \multirow{2}{*}{13} & $\sigma_{t}(\mathrm{pb})$ & $136 \pm 5$ & $720 \pm 12$ & $259 \pm 18$ & $126 \pm 12$ \\
& $\sigma_{\bar{t}}(\mathrm{pb})$ & $81.0_{-3.6}^{+4.1}$ & $265 \pm 11$ & $200 \pm 14$ & $88 \pm 2.4$ \\
\hline
\end{tabular}

Table 4. $t$-channel single-top cross section in the presence of $\xi_{t j}$.

We computed the corrections of the operators $\xi_{t j}$ to the $t$-channel single top cross section in the 5-flavor scheme, including NLO QCD effects building upon the POWHEG implementation in ref. [66]. The right-handed current operators interfere with the SM through terms proportional to the mass of the light down-type quark. For $\xi_{t d}$ and $\xi_{t s}$ operators it is safe to neglect these terms considering the current collider sensitivity. For $\xi_{t b}$, the interference is more important but can also be neglected at present.

In table 4 we give the SM cross section at $\sqrt{S}=7,8$, and $13 \mathrm{TeV}$, and the corrections induced by the RHCC operators. The cross sections are evaluated at the factorization and renormalization scale $\mu=m_{t}$. The scale uncertainty was estimated by varying the factorization and renormalization scales between $\mu=m_{t} / 2$ and $\mu=2 m_{t}$. The PDF and $\alpha_{s}$ uncertainties were conservatively estimated following the original PDF4LHC recipe [67] using the three PDF sets CT10 [68], MSTW08 [69], and NNPDF2.3 [70]. PDF uncertainties turn out to dominate the theoretical uncertainty.

In addition to the single-top production cross section, modifications of the $W t q$ vertex strongly affect the decay of the top quark. An observable particularly sensitive to RHCC is the ratio of the top decay width into a $W$ and a $b$ quark and the width into all down-type quarks. This ratio is measured to be [71]

$$
\frac{\Gamma(t \rightarrow W b)}{\sum_{q=d, s, b} \Gamma(t \rightarrow W q)}=0.957 \pm 0.034
$$

In the presence of $\xi_{t j}$, neglecting terms proportional to $m_{b}$, this becomes

$$
\frac{\Gamma(t \rightarrow W b)}{\sum_{q=d, s, b} \Gamma(t \rightarrow W q)}=\frac{\left|V_{t b}\right|^{2}+\left|\xi_{t b}\right|^{2}}{\sum_{q=d, s, b}\left(\left|V_{t q}\right|^{2}+\left|\xi_{t q}\right|^{2}\right)} .
$$

Additional information is carried by the helicity fractions of $W$ bosons produced from top quark decays, which are mostly sensitive to $\xi_{t b}$. The helicity fractions have been measured at the Tevatron [72] and at the LHC [73-77]. In table 5 we summarize the results used in our analysis. The experimental uncertainty is obtained by combining in quadrature the statistical and systematic errors reported by the experimental collaborations, and considering the correlations between $F_{0}$ and $F_{L, R}$ in the determination of the $\chi^{2}$ function. 


\begin{tabular}{|c|c|c|cc|}
\hline$F_{0}$ & $F_{L}$ & $F_{R}$ & \multicolumn{2}{|c|}{ experiment } \\
\hline $0.72 \pm 0.08$ & $0.31 \pm 0.09$ & $-0.03 \pm 0.04$ & CDF \& D0 & {$[72]$} \\
$0.67 \pm 0.07$ & $0.32 \pm 0.04$ & $0.01 \pm 0.04$ & ATLAS & {$[73]$} \\
$0.68 \pm 0.04$ & $0.31 \pm 0.03$ & $0.01 \pm 0.01$ & CMS & {$[74]$} \\
$0.72 \pm 0.06$ & $0.30 \pm 0.04$ & $-0.02 \pm 0.02$ & CMS & {$[76]$} \\
$0.709 \pm 0.019$ & $0.299 \pm 0.015$ & $0.008 \pm 0.014$ & ATLAS & {$[77]$} \\
\hline
\end{tabular}

Table 5. $W$ helicity fractions measured at CDF, D0, ATLAS, and CMS.

The SM helicity fractions have been computed at NNLO in QCD [78]. They can be expressed as functions of the ratio $x=m_{W} / m_{t}$. Choosing the top pole mass $m_{t}=173 \mathrm{GeV}$ and $m_{W}=80.4 \mathrm{GeV}$, the SM helicity fractions at NNLO are $F_{0}=0.687, F_{L}=0.311$, and $F_{R}=0.0017$. The theoretical uncertainty is very small, only at the permille level, and is negligible compared to the experimental uncertainty. The corrections to the helicity fractions induced by a right-handed $W t b$ vertex have been computed at NLO in QCD in ref. [79], and they can be obtained from the SM by exchanging $F_{R}$ and $F_{L}$.

Combining the information from single-top production, top decay, and $W$ boson helicity fractions, we obtain the following $90 \%$ C.L. bounds

$$
\left|\xi_{t d}\right|<0.13, \quad\left|\xi_{t s}\right|<0.22, \quad\left|\xi_{t b}\right|<0.16
$$

where, as in the previous sections, we assumed that the SM is modified only by a RHCC.

\subsection{Higgs branching ratios}

RHCC affect Higgs decays at tree level, by modifying the $h \rightarrow W W^{*}$ channel, and, more importantly, contributing at one loop to the Yukawa couplings of the Higgs boson to fermions. In particular, $\xi_{t b}$ induces large corrections to the bottom Yukawa coupling $y_{b}$. The corrections are proportional to the top Yukawa coupling $y_{t}$ and alter the $h \rightarrow b \bar{b}$ width and thereby the total Higgs width as well. Defining the quark Yukawa couplings as

$$
\mathcal{L} \supset-y_{q} \bar{q}_{L} q_{R} h+\text { h.c. }
$$

the running of the bottom Yukawa and mass are modified by $\xi_{t b}$ as follows [42, 43, 80],

$$
\begin{aligned}
\frac{d m_{b}}{d \ln \mu} & =\gamma_{m} \frac{\alpha_{s}}{4 \pi} m_{b}+\frac{g^{2}}{(4 \pi)^{2}} m_{t}\left(x_{t}-3\right) V_{t b}^{*} \xi_{t b}, \\
\frac{d y_{b}}{d \ln \mu} & =\gamma_{m} \frac{\alpha_{s}}{4 \pi} y_{b}+\frac{g^{2}}{(4 \pi)^{2}} \frac{m_{t}}{v}\left(x_{h}+3 x_{t}-9\right) V_{t b}^{*} \xi_{t b},
\end{aligned}
$$

where $x_{i}=m_{i}^{2} / m_{W}^{2}$. The anomalous dimension $\gamma_{m}=-6 C_{F}$ determines the usual one-loop evolution from QCD effects, while the $\xi_{t b}$ terms alter the evolution between the scale of new physics, $\Lambda$, and $\mu=M_{W}$. We use the boundary condition $y_{b}(\Lambda)=m_{b}(\Lambda) / v$, which 
follows from our assumption that the $\xi$ operators are the only dimension-six terms present at $\mu=\Lambda$. In this case the SM Yukawa couplings are not modified at this scale.

Apart from these RG effects, the right-handed current operators affect the $h \rightarrow b \bar{b}$ process through finite loop contributions. The effective coupling that is probed in Higgs decays, is then given by

$$
\begin{aligned}
y_{b}^{(\mathrm{eff})}=y_{b}(\mu)-\frac{g^{2}}{(4 \pi)^{2}} \frac{m_{t}}{v} \frac{\xi_{t b} V_{t b}^{*}}{2}[ & \left(x_{h}+3 x_{t}-9\right) \log \frac{\mu^{2}}{m_{W}^{2}} \\
& -3 \frac{x_{t}\left(x_{t}-3\right)}{x_{t}-1} \log x_{t}+x_{t} \beta_{t} \log \left(\frac{\beta_{t}-1}{\beta_{t}+1}\right) \\
& +\left(x_{h}-2\right) \beta_{w} \log \left(\frac{\beta_{W}-1}{\beta_{W}+1}\right)+\left(2 x_{h}+x_{t}\left(x_{t}-7\right)\right) f_{1}\left(x_{h}, x_{t}\right) \\
& \left.-\left(4+\left(2-x_{h}\right) x_{t}\right) f_{2}\left(x_{h}, x_{t}\right)+\left(-5+2 x_{h}+4 x_{t}\right)\right],
\end{aligned}
$$

where $y_{b}(\mu)$ is given by the solution of eq. (2.20), $\beta_{i}=\sqrt{1-4 m_{i}^{2} / m_{h}^{2}}$, and the two functions $f_{1,2}$ are given by

$$
\begin{aligned}
& f_{1}\left(x_{h}, x_{t}\right)=\int_{0}^{1} d z \frac{1}{1-x_{t}+x_{h} z} \log \left(\frac{x_{t}-x_{h}(1-z) z}{1+\left(x_{t}-1\right) z}\right), \\
& f_{2}\left(x_{h}, x_{t}\right)=\int_{0}^{1} d z \frac{1}{-1+x_{t}+x_{h} z} \log \left(\frac{1-x_{h}(1-z) z}{x_{t}+z\left(1-x_{t}\right)}\right) .
\end{aligned}
$$

Setting $\mu=m_{H}$ and $\Lambda=1 \mathrm{TeV}$, we find numerically $y_{b}^{(\text {eff })} \simeq 0.012-0.019 \xi_{t b} V_{t b}^{*}$. We then compute the $h \rightarrow b \bar{b}$ decay rate using expressions in ref. [81]. Using the ATLAS and CMS combination [41] for the $\gamma \gamma, W W, Z Z, \tau \tau, b \bar{b}$, and $\mu \mu$ signal strengths, we obtain,

$$
\operatorname{Re} \xi_{t b} \in[-0.01,0.13], \quad \operatorname{Im} \xi_{t b} \in[-0.22,0.22],
$$

where we turned on only one parameter, the real or imaginary part, at the time, and the bounds assume $\Lambda=1 \mathrm{TeV}$. The limits are only mildly sensitive to the choice of initial scale $\Lambda$. Setting, for example, $\Lambda=10 \mathrm{TeV}$ results in $\operatorname{Re} \xi_{t b} \in[-0.005,0.10]$, and $\operatorname{Im} \xi_{t b} \in$ $[-0.15,0.15]$. Notice that the bounds from $h \rightarrow b \bar{b}$ are already competitive with the direct bounds from single-top production and top decay.

Analogously to $\xi_{t b}$, the $\xi_{t d}$ and $\xi_{t s}$ elements give contributions to the down- and strangequark Yukawas that are enhanced by $y_{t}$, with, in this case, some suppression from the small CKM elements $V_{t d}$ and $V_{t s}$. Since, at the moment, $y_{d}$ and $y_{s}$ are constrained at the same level as $y_{b}[82,83]$, the bounds on $\xi_{t d}$ and $\xi_{t s}$ are weaker than eq. (2.23) and not competitive with single top production and top decay. The contributions of the first two rows of the $\xi_{i j}$ matrix to the quark Yukawa couplings are not enhanced by the top Yukawa. In this case, tree level corrections to $h \rightarrow W W^{*}$ are more important. Neglecting quark and lepton masses, the decay rate at $\mathrm{LO}$ in QCD is equal to

$$
\Gamma\left(h \rightarrow W W^{*}\right)=\frac{3 m_{H} m_{W}^{4}}{32 \pi^{3} v^{4}}\left(R\left(x_{W}\right)+\sum_{i, j}\left(\left|V_{i j}\right|^{2} R\left(x_{W}\right)+\left|\xi_{i j}\right|^{2} R_{\xi}\left(x_{W}\right)\right)\right)
$$


where $x_{W}=m_{W}^{2} / m_{H}^{2}$, the sum over $i$ and $j$ extends over all light quarks, and the functions $R$ and $R_{\xi}$ are [81]

$$
\begin{aligned}
R(x)= & -\frac{1-x}{6 x}\left(2-13 x+47 x^{2}\right)-\frac{1}{2}\left(1-6 x+4 x^{2}\right) \log x+\frac{\left(1-8 x+20 x^{2}\right)}{\sqrt{4 x-1}} \arccos \left(\frac{3 x-1}{2 x^{3 / 2}}\right) \\
R_{\xi}(x)= & -\frac{1-x}{36 x^{2}}\left(-3+53 x-541 x^{2}+407 x^{3}\right)+\frac{1}{6 x}\left(2+3 x+114 x^{2}-12 x^{3}\right) \log x \\
& +\frac{-2+19 x-80 x^{2}+156 x^{3}}{3 x \sqrt{4 x-1}} \arccos \left(\frac{3 x-1}{2 x^{3 / 2}}\right) .
\end{aligned}
$$

For $m_{H}=125 \mathrm{GeV}, R_{\xi}\left(x_{W}\right) / R\left(x_{W}\right)=0.03$, such that

$$
\Gamma\left(h \rightarrow W W^{*}\right)=\Gamma_{\mathrm{SM}}\left(h \rightarrow W W^{*}\right)\left(1+0.01 \sum_{i j}\left|\xi_{i j}\right|^{2}\right),
$$

and very large couplings, $\xi_{i j}>1$, are needed to significantly affect the Higgs branching ratios.

\subsection{Summary of collider bounds}

To summarize, the $90 \%$ C.L. bounds on the $\xi_{i j}$ matrix are

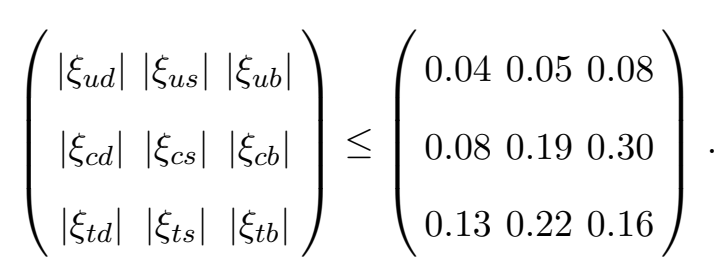

The bounds on the first two rows are dominated by $W H$ production, while the bounds on $\xi_{t j}$ are determined by single-top production and top decay. Including the constraint from $h \rightarrow b \bar{b}$ changes the bounds on $\xi_{t b}$ into $\operatorname{Re} \xi_{t b} \in[-0.01,0.13]$ and $\operatorname{Im} \xi_{t b} \in[-0.15,0.15]$. The above collider constraints still leave room for BSM physics around, or even slightly below, the $\mathrm{TeV}$ scale.

\section{Low-energy effective Lagrangian}

The effects of the RHCC operator $\xi$ at low energies are obtained by integrating out the $W$ boson and the other heavy SM degrees of freedom. We start by analyzing the tree-level contributions to semileptonic and four-quark operators and then discuss the loop-level operators that are relevant for EDMs $(\Delta F=0)$ and rare flavor-changing neutral-current (FCNC) processes such as $B \rightarrow X_{q} \gamma$ and $K_{L} \rightarrow \pi^{0} e^{+} e^{-}(\Delta F=1)$. The matching onto $\Delta F=1$ penguin operators and $\Delta F=2$ operators relevant for meson-antimeson mixing, which involve two insertions of $\xi$, are discussed in appendix A. A similar analysis for SMEFT operators involving the $\mathrm{Z}$ boson was recently reported in ref. [84]. 


\subsection{Tree-level effective Lagrangian}

The combination of the SM left-handed charged current and the RHCC generates at lowenergy one semileptonic four-fermion and two four-quark operators. Including the fourfermion operators induced by the SM at tree level, we have

$$
\begin{aligned}
\mathcal{L}= & -\frac{4 G_{F}}{\sqrt{2}}\left(V_{i j}^{*} \bar{d}^{j} \gamma^{\mu} P_{L} u^{i} \bar{\nu} \gamma_{\mu} P_{L} l+\xi_{i j}^{*} \bar{d}^{j} \gamma^{\mu} P_{R} u^{i} \bar{\nu} \gamma_{\mu} P_{L} l+\text { h.c. }\right) \\
& -\sum_{a=1}^{2}\left(C_{a L L}^{i j l m} \mathcal{O}_{a L L}^{i j l m}+C_{a L R}^{i j l m} \mathcal{O}_{a L R}^{i j l m}+C_{a L R}^{i j l m *}\left(\mathcal{O}_{a L R}^{i j l m}\right)^{\dagger}\right),
\end{aligned}
$$

where $P_{L, R}=\left(1 \mp \gamma_{5}\right) / 2, i-m$ are flavor indices, and the four-quark operators are defined as

$$
\begin{array}{ll}
\mathcal{O}_{1 L L}^{i j l m}=\bar{d}^{m} \gamma^{\mu} P_{L} u^{l} \bar{u}^{i} \gamma_{\mu} P_{L} d^{j}, & \mathcal{O}_{2 L L}^{i j l m}=\bar{d}_{\alpha}^{m} \gamma^{\mu} P_{L} u_{\beta}^{l} \bar{u}_{\beta}^{i} \gamma_{\mu} P_{L} d_{\alpha}^{j}, \\
\mathcal{O}_{1 L R}^{i j l m}=\bar{d}^{m} \gamma^{\mu} P_{L} u^{l} \bar{u}^{i} \gamma_{\mu} P_{R} d^{j}, & \mathcal{O}_{2 L R}^{i j l m}=\bar{d}_{\alpha}^{m} \gamma^{\mu} P_{L} u_{\beta}^{l} \bar{u}_{\beta}^{i} \gamma_{\mu} P_{R} d_{\alpha}^{j},
\end{array}
$$

where $\alpha, \beta$ are color indices. The tree-level matching coefficients at the scale $m_{W}$ are given by

$$
C_{1 L L}^{i j l m}\left(m_{W}\right)=\frac{4 G_{F}}{\sqrt{2}} V_{l m}^{*} V_{i j}, \quad C_{1 L R}^{i j l m}\left(m_{W}\right)=\frac{4 G_{F}}{\sqrt{2}} V_{l m}^{*} \xi_{i j}, \quad C_{2 A B}^{i j l m}\left(m_{W}\right)=0,
$$

where $A, B \in\{L, R\}$ and hermiticity implies $C_{1 L L}^{l m i j *}=C_{1 L L}^{i j l m}$. As usual, the SM couplings scale as two inverse powers of the electroweak scale, $C_{i L L} \sim 1 / v^{2}$, while the 'left-right' operators induced by the RHCC scale as two inverse powers of the scale of new physics, $C_{i L R} \sim \xi / v^{2} \sim 1 / \Lambda^{2}$. We neglect four-quark operators that are quadratic in $\xi$ and are suppressed by $v^{2} / \Lambda^{2}$ with respect to the linear terms.

The operators in eq. (3.1) need to be evolved to lower energies. While the semileptonic operators are not affected by QCD RG evolution, the anomalous dimensions of the fourquark operators are defined by $[85,86]$

$$
\frac{d}{d \log \mu}\left(C_{1 A B}, C_{2 A B}\right)^{T}=\frac{\alpha_{s}}{4 \pi} \sum_{n=0}\left(\frac{\alpha_{s}}{4 \pi}\right)^{n} \gamma_{A B}^{(n)}\left(C_{1 A B}, C_{2 A B}\right)^{T},
$$

and, at lowest order, we have

$$
\gamma_{L L}^{(0)}=\left(\begin{array}{cc}
-\frac{6}{N_{c}} & 6 \\
6 & -\frac{6}{N_{c}}
\end{array}\right), \quad \gamma_{L R}^{(0)}=\left(\begin{array}{cc}
\frac{6}{N_{c}} & 0 \\
-6 & -6 \frac{N_{c}^{2}-1}{N_{c}}
\end{array}\right) .
$$

The solution of the RGE for the operators of interest is given in table 7 .

The semileptonic operators in eq. (3.1) affect leptonic and semileptonic decays of mesons and the $\beta$ decay of baryons. In particular, $\xi$ is the only dimension-six operator in the SMEFT that induces a tree-level charged-current coupling of right-handed quarks to left-handed leptons, allowing for clean low-energy tests. The coefficients of the four-quark operators $\mathcal{O}_{i L R}$ have an imaginary part which leads to $\mathrm{CP}$ violation even if all generation indices are the same. They therefore induce large tree-level contributions to observables such as EDMs and $\epsilon^{\prime} / \epsilon$. 


\subsection{One-loop contributions to $\Delta F=0$ and $\Delta F=1$ operators}

Next we consider loop diagrams, which can induce important contributions to processes such as $B \rightarrow X_{q} \gamma, K_{L} \rightarrow \pi^{0} e^{+} e^{-}$and to EDMs. At linear order in $\xi$, the most important operators that are generated are dipole operators and the Weinberg three-gluon operator, described by

$$
\begin{aligned}
\mathcal{L}= & \left(-\frac{g_{s}}{2} \sum_{i j \in\{u, c\}} m_{u_{j}} C_{g u}^{i j} \bar{u}_{L}^{i} \sigma^{\mu \nu} G_{\mu \nu}^{a} t^{a} u_{R}^{j}-\frac{g_{s}}{2} \sum_{i j \in\{d, s, b\}} m_{d_{j}} C_{g d}^{i j} \bar{d}_{L}^{i} \sigma^{\mu \nu} G_{\mu \nu}^{a} t^{a} d_{R}^{j}\right. \\
& -\frac{e Q_{u}}{2} \sum_{i j \in\{u, c\}} m_{u_{j}} C_{\gamma u}^{i j} \bar{u}_{L}^{i} \sigma^{\mu \nu} F_{\mu \nu} u_{R}^{j}-\frac{e Q_{d}}{2} \sum_{i j \in\{d, s, b\}} m_{d_{j}} C_{\gamma d}^{i j} \bar{d}_{L}^{i} \sigma^{\mu \nu} F_{\mu \nu} d_{R}^{j} \\
& \left.-\frac{e Q_{e}}{2} \sum_{i j \in\{e, \mu, \tau\}} m_{e_{j}} C_{\gamma l}^{i j} \bar{e}_{L}^{i} \sigma^{\mu \nu} F_{\mu \nu} e_{R}^{j}+\text { h.c. }\right)+\frac{g_{s}}{3} f^{a b c}\left(C_{G} G^{a \mu \nu}+\frac{C_{\tilde{G}}}{2} \varepsilon^{\mu \nu \alpha \beta} G_{\alpha \beta}^{a}\right) G_{\mu \rho}^{b} G_{\nu}^{c \rho},
\end{aligned}
$$

where we chose to factor the mass of the right-handed quark or lepton out of the definition of the dipole operators. The lepton dipoles receive a contribution from $\xi_{t b}$ at two loops, which, neglecting neutrino mass effects, is diagonal in lepton flavor. We discuss this contribution in appendix B. The operators in eq. (3.6) satisfy the RGEs

$$
\begin{aligned}
\frac{d}{d \log \mu}\left(C_{\gamma u}^{i j}, C_{g u}^{i j}, C_{G}+i C_{\tilde{G}}\right)^{T}= & \frac{\alpha_{s}}{4 \pi} \sum_{n}\left(\frac{\alpha_{s}}{4 \pi}\right)^{n} \gamma_{\text {dipole }}^{(n)}\left(C_{\gamma u}^{i j}, C_{g u}^{i j}, C_{G}+i C_{\tilde{G}}\right)^{T} \\
& +\frac{\alpha_{s}}{4 \pi} \frac{m_{d_{m}}}{m_{u_{j}}} \sum_{n}\left(\frac{\alpha_{s}}{4 \pi}\right)^{n} \gamma_{\text {dipole, uLR }}^{(n)}\left(C_{1 L R}^{j m i m *}, C_{2 L R}^{j m i m *}\right)^{T} \\
\frac{d}{d \log \mu}\left(C_{\gamma d}^{i j}, C_{g d}^{i j}, C_{G}+i C_{\tilde{G}}\right)^{T}= & \frac{\alpha_{s}}{4 \pi} \sum_{n}\left(\frac{\alpha_{s}}{4 \pi}\right)^{n} \gamma_{\text {dipole }}^{(n)}\left(C_{\gamma d}^{i j}, C_{g d}^{i j}, C_{G}+i C_{\tilde{G}}\right)^{T} \\
& +\frac{\alpha_{s}}{4 \pi} \frac{m_{u_{m}}}{m_{d_{j}}} \sum_{n}\left(\frac{\alpha_{s}}{4 \pi}\right)^{n} \gamma_{\text {dipole, dLR }}^{(n)}\left(C_{1 L R}^{m j m i}, C_{2 L R}^{m j m i}\right)^{T} .
\end{aligned}
$$

At lowest order $\gamma_{\text {dipole }}$ is given by [86-89]

$$
\gamma_{\text {dipole }}^{(0)}=\left(\begin{array}{ccc}
8 C_{F} & -8 C_{F} & 0 \\
0 & \left(16 C_{F}-4 N_{c}\right) & 2 N_{c} \delta_{i j} \\
0 & 0 & N_{c}+2 n_{f}+\beta_{0}
\end{array}\right) .
$$

The mixing between the tree-level operators $C_{1,2} L R$ and the dipole operators was computed in ref. [90] for down-type dipoles, and it is given by $[90,91]^{2}$

$$
\gamma_{\text {dipole,dLR }}^{(0)}=\frac{1}{(4 \pi)^{2}}\left(\begin{array}{cc}
32 \frac{Q_{u}}{Q_{d}}\left(1+\frac{2}{3} \frac{Q_{d}}{Q_{u}}\right) & 160 \frac{Q_{u}}{Q_{d}} \\
\frac{16}{3} & -8 \\
0 & 0
\end{array}\right) .
$$

$\gamma_{\text {dipole, uLR }}^{(0)}$ is obtained by replacing $Q_{d}$ with $Q_{u}$ in eq. (3.10).

\footnotetext{
${ }^{2}$ We thank M. Misiak for providing us the expression of $\gamma_{\text {dipole,dLR }}^{(0)}$ for general charges $Q_{d}$ and $Q_{u}[91]$.
} 


\begin{tabular}{|c|ccc|}
\hline$q^{\prime} \rightarrow q \gamma$ & $\xi_{u q^{\prime}} V_{u q}^{*}$ & $\xi_{c q^{\prime}} V_{c q}^{*}$ & $\xi_{t q^{\prime}} V_{t q}^{*}$ \\
\hline$\frac{m_{q^{\prime}}}{m_{b}} v^{2} C_{\gamma d}^{q q^{\prime}}\left(m_{W}\right)$ & $-3.6 \cdot 10^{-5}$ & -0.019 & -3.2 \\
$\frac{m_{q^{\prime}}}{m_{b}} v^{2} C_{g d}^{q q^{\prime}}\left(m_{W}\right)$ & - & - & -0.48 \\
\hline$\frac{m_{q^{\prime}}}{m_{b}} v^{2} C_{\gamma d}^{q q^{\prime}}\left(\mu_{b}^{+}\right)$ & $-4.7 \cdot 10^{-5}$ & -0.025 & -2.0 \\
$\frac{m_{q^{\prime}}}{m_{b}} v^{2} C_{g d}^{q q^{\prime}}\left(\mu_{b}^{+}\right)$ & $-8.0 \cdot 10^{-7}$ & $-4.3 \cdot 10^{-4}$ & -0.30 \\
\hline$\frac{m_{q^{\prime}}}{m_{b}} v^{2} C_{\gamma d}^{q q^{\prime}}\left(\mu_{b}^{-}\right)$ & $-4.7 \cdot 10^{-5}$ & -0.054 & -2.0 \\
$\frac{m_{q^{\prime}}}{m_{b}} v^{2} C_{g d}^{q q^{\prime}}\left(\mu_{b}^{-}\right)$ & $-8.0 \cdot 10^{-7}$ & $-6.2 \cdot 10^{-3}$ & -0.30 \\
\hline
\end{tabular}

Table 6. Contributions of the right-handed $W$-current to the $q^{\prime} \rightarrow q \gamma$ dipole operators at $\mu=m_{W}$ and $\mu=\mu_{b}^{ \pm}=2 \mathrm{GeV}$. Here $\mu_{b}^{-}$and $\mu_{b}^{+}$differentiate between cases in which the charm quark has been integrated out at $\mu=\mu_{b}$, or is still present within the EFT, respectively. A " - " denotes the contribution is negligible for our purposes.

The one-loop matching coefficients at the scale $m_{W}$ are

$$
\begin{aligned}
C_{\gamma u}^{i l}\left(m_{W}\right) & =\frac{4 G_{F}}{\sqrt{2}} \frac{2}{(4 \pi)^{2}} \sum_{j \in\{d, s, b\}} \frac{m_{d_{j}}}{m_{u_{l}} Q_{u}} \xi_{l j}^{*} V_{i j}, \\
C_{g u}^{i l}\left(m_{W}\right) & =0, \\
C_{\gamma d}^{i l}\left(m_{W}\right) & =\frac{4 G_{F}}{\sqrt{2}} \frac{2}{(4 \pi)^{2}} \sum_{j \in\{u, c\}} \frac{m_{u_{j}}}{m_{d_{l}} Q_{d}} \xi_{j l} V_{j i}^{*}+\frac{4 G_{F}}{\sqrt{2}} \frac{1}{(4 \pi)^{2}} \frac{m_{t} Q_{u}}{m_{d_{l}} Q_{d}} \xi_{t l} V_{t i}^{*}\left[f_{W}\left(x_{t}\right)+\frac{1}{Q_{u}} g_{W}\left(x_{t}\right)\right], \\
C_{g d}^{i l}\left(m_{W}\right) & =-\frac{4 G_{F}}{\sqrt{2}} \frac{1}{(4 \pi)^{2}} \frac{m_{t}}{m_{d_{l}}} \xi_{t l} V_{t i}^{*} f_{W}\left(x_{t}\right), \\
C_{\tilde{G}}\left(m_{W}\right) & =0,
\end{aligned}
$$

where $x_{t}=m_{t}^{2} / m_{W}^{2}$, and we neglected powers of $x_{u_{j}}$ and $x_{d_{j}}$ except for the top quark. The loop functions are given by

$$
f_{W}(x)=\frac{x^{3}+3 x-4-6 x \ln x}{2(x-1)^{3}}, \quad g_{W}(x)=\frac{4+x(x-11)}{2(x-1)^{2}}+3 \frac{x^{2} \ln x}{(x-1)^{3}} .
$$

Notice that at the scale $m_{W}$ there is no matching contribution to the Weinberg operator. Two-loop diagrams with internal top and bottom quarks, and a $W$ exchange within the loop, as the ones computed in ref. [92], are exactly canceled by a diagram in the EFT below $m_{W}$, with an insertion of a bottom chromo-EDM (CEDM), the $C_{g d}^{b b}$ operator in eq. (3.11), such that $C_{\tilde{G}}\left(m_{W}\right)=0$.

The operators $C_{\gamma(g) d}^{b q}$ and $C_{\gamma(g) d}^{q b}$ contribute to $B \rightarrow X_{q} \gamma$, and their value at the scales $\mu=m_{W}$ and $\mu=\mu_{b}=2 \mathrm{GeV}$ are given in table 6 . The contributions from diagrams with internal top quarks are enhanced by $m_{t} / m_{b}$ with respect to the SM, which, as we will see, leads to stringent bounds on the $\xi_{t q}$ elements of the right-handed matrix. In order to match 
onto the relevant operators for EDMs and $K_{L} \rightarrow \pi^{0} e^{+} e^{-}$one has to consider the contributions arising at the bottom and charm thresholds. At the scale $m_{b}$, four-quark operators such as $C_{1 L R}^{u b u b}$ generate threshold corrections to the up and charm EDM and CEDMs, while the bottom CEDM contributes to the Weinberg operator. Thus, at the $b$ threshold

$$
\begin{aligned}
C_{\gamma u}^{i l}\left(m_{b}^{-}\right) & =C_{\gamma u}^{i l}\left(m_{b}^{+}\right)+\frac{2}{(4 \pi)^{2}} \frac{m_{b} Q_{d}}{m_{u_{l}} Q_{u}}\left(C_{1 L R}^{l b i b *}\left(m_{b}\right)+N_{c} C_{2 L R}^{l b i b *}\left(m_{b}\right)\right), \\
C_{g u}^{i l}\left(m_{b}^{-}\right) & =-\frac{2}{(4 \pi)^{2}} \frac{m_{b}}{m_{u_{l}}} C_{1 L R}^{l b i b *}\left(m_{b}\right), \\
C_{\tilde{G}}\left(m_{b}^{-}\right) & =-\frac{\alpha_{s}}{8 \pi} \operatorname{Im} C_{g d}^{b b}\left(m_{b}\right),
\end{aligned}
$$

where we took into account that, with the initial condition of eq. (3.11), the running from $m_{W}$ to $m_{b}$ does not induce an up-type CEDM, or a Weinberg operator. There is no $b$-threshold contribution to $C_{g d}^{i l}$ and $C_{\gamma d}^{i l}$.

At a scale $\mu_{c} \sim m_{c}$, one similarly integrates out the charm quark, and obtains additional threshold corrections to the $d$ and $s$ dipoles, and to the Weinberg operator

$$
\begin{aligned}
& C_{\gamma d}^{i l}\left(\mu_{c}^{-}\right)=C_{\gamma d}^{i l}\left(\mu_{c}^{+}\right)+\frac{2}{(4 \pi)^{2}} \frac{m_{c} Q_{u}}{m_{d_{l}} Q_{d}}\left(C_{1 L R}^{c l c i}\left(\mu_{c}\right)+N_{c} C_{2 L R}^{c l c i}\left(\mu_{c}\right)\right) \\
& C_{g d}^{i l}\left(\mu_{c}^{-}\right)=C_{g d}^{i l}\left(\mu_{c}^{+}\right)-\frac{2}{(4 \pi)^{2}} \frac{m_{c}}{m_{d_{l}}} C_{1 L R}^{c l c i}\left(\mu_{c}\right) \\
& C_{\tilde{G}}\left(\mu_{c}^{-}\right)=C_{\tilde{G}}\left(\mu_{c}^{+}\right)-\frac{\alpha_{s}}{8 \pi} \operatorname{Im} C_{g u}^{c c}\left(\mu_{c}\right) .
\end{aligned}
$$

In order to avoid large perturbative corrections, we run to the scale $\mu_{c}=2 \mathrm{GeV}$.

The numerical solution of the RGEs is shown in table 7 , where, for convenience, we introduced the short-hand notation $\tilde{c}_{i}=v^{2} \operatorname{Im} C_{i}$. As the RHCC operator does not undergo QCD renormalization, the results in table 7, as those in table 6, are independent of $\Lambda$ to good approximation. Some of these results, especially the contributions of $\xi_{u b, c b, t b}$ to the light quark EDMs, are rather sensitive to the choice of renormalization scale. This effect is due to a partial cancellation between matching contributions and contributions from the CEDMs. In these cases, however, the largest contributions to hadronic EDMs come from other operators $\left(\tilde{c}_{g u}^{u u}\right.$ for $\xi_{u b}$ and $C_{\tilde{G}}$ for $\left.\xi_{c b, t b}\right)$ that are less sensitive to the details of the running such that the impact of perturbative uncertainties is still minor. We expect the large hadronic uncertainties related to these operators, which we discuss in section 5 , to dominate the theoretical uncertainties on hadronic EDMs.

\section{Leptonic and semileptonic charged-current decays}

The right-handed current matrix $\xi_{i j}$ is strongly constrained by leptonic and semileptonic meson decays, and semileptonic decays of baryons. Leptonic decays of pseudoscalar mesons, such as $\pi^{+} \rightarrow \mu^{+} \nu_{\mu}$ or $D^{+} \rightarrow \mu^{+} \nu_{\mu}$, are sensitive to the axial component of the weak current, while semileptonic decays of pseudoscalar mesons into pseudoscalar mesons and leptons, such as $K \rightarrow \pi l \nu_{l}$, probe the vector component. For the $B$ system, one can in addition study semileptonic decays of pseudoscalar to vector mesons, such as $B \rightarrow D^{*} l \nu_{l}$, 


\begin{tabular}{|c|ccccccccc|}
\hline & $V_{u d}^{*} \xi_{u d}$ & $V_{u s}^{*} \xi_{u s}$ & $V_{u b}^{*} \xi_{u b}$ & $V_{c d}^{*} \xi_{c d}$ & $V_{c s}^{*} \xi_{c s}$ & $V_{c b}^{*} \xi_{c b}$ & $V_{t d}^{*} \xi_{t d}$ & $V_{t s}^{*} \xi_{t s}$ & $V_{t b}^{*} \xi_{t b}$ \\
\hline$\tilde{c}_{\gamma l}^{\text {ee }}$ & - & - & - & - & - & - & - & - & $6.8 \times 10^{-6}$ \\
$\tilde{c}_{\gamma u}^{u u}$ & -0.033 & -0.65 & 7.1 & - & - & $-1.7 \cdot 10^{-8}$ & - & - & $-1.5 \cdot 10^{-5}$ \\
$\tilde{c}_{g u}^{u u}$ & $3.6 \cdot 10^{-3}$ & 0.073 & 47 & - & - & $-2.3 \cdot 10^{-7}$ & - & - & $-2.0 \cdot 10^{-4}$ \\
$\tilde{c}_{\gamma d}^{d d}$ & -0.047 & - & - & -54 & - & $-1.7 \cdot 10^{-8}$ & -2029 & - & $-1.5 \cdot 10^{-5}$ \\
$\tilde{c}_{g d}^{d d}$ & $-8.0 \cdot 10^{-4}$ & - & - & -6.2 & - & $-2.3 \cdot 10^{-7}$ & -298 & - & $-2.0 \cdot 10^{-4}$ \\
$\tilde{c}_{\gamma d}^{s s}$ & - & $-2.3 \cdot 10^{-3}$ & - & - & -2.7 & $-1.7 \cdot 10^{-8}$ & - & -102 & $-1.5 \cdot 10^{-5}$ \\
$\tilde{c}_{g d d}^{s s}$ & - & $-4.0 \cdot 10^{-5}$ & - & - & -0.31 & $-2.3 \cdot 10^{-7}$ & - & -15 & $-2.0 \cdot 10^{-4}$ \\
$v^{2} C_{\tilde{G}}$ & - & - & - & - & - & $-1.2 \cdot 10^{-3}$ & - & - & $2.2 \cdot 10^{-3}$ \\
$\tilde{C}_{1 L R}^{u d u d}$ & 1.8 & - & - & - & - & - & - & - & - \\
$\tilde{C}_{2 L R}^{\text {ud ud }}$ & 0.91 & - & - & - & - & - & - & - & - \\
$\tilde{C}_{1 L R}^{u s u s}$ & - & 1.8 & - & - & - & - & - & - & - \\
$\tilde{C}_{2 L R}^{u s u s}$ & - & 0.91 & - & - & - & - & - & - & - \\
\hline
\end{tabular}

Table 7. Contributions of the CP-odd combinations, $\operatorname{Im}\left(V_{i j}^{*} \xi_{i j}\right)$, to the operators at $\mu=2 \mathrm{GeV}$. Here $\tilde{c}_{i} \equiv v^{2} \operatorname{Im} C_{i}$ and a " $-"$ denotes that the contribution is negligible for our purposes.

\begin{tabular}{|c|c|c|c|}
\hline & Decay constant & & Form Factor \\
\hline$f_{\pi}$ & $130.2 \pm 1.4 \mathrm{MeV}$ & $f_{+}^{K \pi}(0)$ & $0.9677 \pm 0.0027$ \\
$f_{K} / f_{\pi}$ & $1.192 \pm 0.005$ & & \\
$f_{D}$ & $209.2 \pm 3.3 \mathrm{MeV}$ & $f_{+}^{D \pi}(0)$ & $0.666 \pm 0.029$ \\
$f_{D_{s}}$ & $249.8 \pm 2.3 \mathrm{MeV}$ & $f_{+}^{D K}(0)$ & $0.747 \pm 0.019$ \\
$f_{B}$ & $192.0 \pm 4.3 \mathrm{MeV}$ & $\mathcal{F}_{D}(1)$ & $1.035 \pm 0.040$ \\
$f_{B_{s}}$ & $228.4 \pm 3.7 \mathrm{MeV}$ & $\mathcal{F}_{D^{*}}(1)$ & $0.906 \pm 0.004 \pm 0.012$ \\
\hline
\end{tabular}

Table 8. Lattice input on pseudoscalar meson decay constants and form factors. We use the FLAG lattice averages with $n_{f}=2+1$ [93].

and further orthogonal information is provided by inclusive $B$ decays, $B \rightarrow X_{u, c} l \nu_{l}$. $\beta$ decays of heavy and light baryons, such as $n \rightarrow p e^{-} \bar{\nu}$ or $\Lambda_{b} \rightarrow \Lambda_{c} \mu^{-} \bar{\nu}$, give, in principle, a direct handle on the chiral structure of the interactions, and allow one to construct observables that are sensitive to $\mathrm{CP}$ violation in the matrix $\xi$. An example is the triple correlation, $D_{n}$, measured in neutron $\beta$ decay.

From the theoretical point of view, leptonic and semileptonic decays are very clean observables. Leptonic decays are characterized by a single nonperturbative parameter, the meson decay constant, whose values are nowadays precisely computed with lattice QCD 
(LQCD) [93]. Semileptonic transitions have also been the subject of intense lattice study, and the required form factors are known to high accuracy. In table 8 we list the values of the pseudoscalar meson decay constants and form factors that we use in our analysis. The LQCD input has been taken from the FLAG review [93].

We now list the experimental information we use to constrain the elements of the matrix $\xi$.

$\boldsymbol{u} \rightarrow \boldsymbol{d}$ and $\boldsymbol{u} \rightarrow \boldsymbol{s}$ transitions. $V_{u d}$ is extracted from superallowed nuclear $\beta$ decay, which is only sensitive to the vector component of the weak current, and from leptonic decays of the pion, which probe the axial component of the current. We use the following experimental input $[71,94]$

$$
\begin{aligned}
\left|V_{u d}\left(0^{+} \rightarrow 0^{+}\right)\right|_{\exp } & =0.97425 \pm 0.00022, \\
\left|V_{u d}(\pi \rightarrow \mu \nu) f_{\pi}\right|_{\exp } & =(127.13 \pm 0.02 \pm 0.13) \mathrm{MeV},
\end{aligned}
$$

where $f_{\pi} \sim 130 \mathrm{MeV}$ is the pion decay constant. The first uncertainty in the second line of eq. (4.1) is experimental, while the second is due to radiative corrections.

For the determination of $V_{u s}$, we use two quantities that are experimentally very well determined [95, 96]. From semileptonic kaon decays, one can extract

$$
\left(\left|V_{u s}\right| f_{+}^{K \pi}(0)\right)_{\exp }=0.2163 \pm 0.0005,
$$

where $f_{+}^{K \pi}(0)$ is the form factor entering the $K^{0} \rightarrow \pi^{-} l \nu_{l}$ decay at zero momentum transfer. The ratio of the pion and kaon leptonic decays gives

$$
\left(\left|\frac{V_{u s}}{V_{u d}}\right| \frac{f_{K}}{f_{\pi}}\right)_{\exp }=0.2758 \pm 0.0005 .
$$

Because leptonic and semileptonic decays are sensitive to either the axial or the vector components of the current, we can easily modify the relevant expressions in the presence of a RHCC to obtain

$$
\begin{aligned}
\left|V_{u d}+\xi_{u d}\right| & =0.97425 \pm 0.00022, & & \left|V_{u d}-\xi_{u d}\right| f_{\pi}=(127.13 \pm 0.02 \pm 0.13) \mathrm{MeV} \\
\left|V_{u s}+\xi_{u s}\right| f_{+}^{K \pi}(0) & =0.2163 \pm 0.0005, & & \frac{\left|V_{u s}-\xi_{u s}\right| f_{K}}{\left|V_{u d}-\xi_{u d}\right| f_{\pi}}=0.2758 \pm 0.0005
\end{aligned}
$$

Using the LQCD input in table 8, eq. (4.4) provides four constraints on $V_{u d}, V_{u s}, \xi_{u d}$, and $\xi_{u s}$. Two additional constraints on the imaginary part of $\xi_{u d}$ and $\xi_{u s}$ can be obtained from neutron and hyperon $\beta$ decays. Time-reversal violation can be measured in neutron $\beta$ decay by reconstructing the triple correlation $\langle\vec{J}\rangle \cdot\left(\vec{p}_{e} \times \vec{p}_{\nu}\right)$, where $\vec{J}$ is the neutron polarization. Current measurements give $D_{n}=(-1 \pm 2.1) \cdot 10^{-4}$ [97]. This observable is contaminated by fake $T$-odd signals from final-state interactions, which, with current experimental accuracy, can still be neglected (see ref. [98] for a more detailed discussion). The same correlation was measured in the decay of the $\Sigma$ baryon, $\Sigma^{-} \rightarrow n e^{-} \bar{\nu}$, with a much weaker bound, $D_{\Sigma}=0.11 \pm 0.10$. Following ref. [99], the $D_{n}$ and $D_{\Sigma}$ coefficients can be calculated as

$$
D_{n}=\frac{4 g_{A}}{1+3 g_{A}^{2}} \operatorname{Im} \frac{\xi_{u d}}{V_{u d}} \simeq 0.87 \operatorname{Im} \frac{\xi_{u d}}{V_{u d}}, \quad D_{\Sigma}=\frac{4 g_{A \Sigma n}}{1+3 g_{A \Sigma n}^{2}} \operatorname{Im} \frac{\xi_{u s}}{V_{u s}} \simeq 1.01 \operatorname{Im} \frac{\xi_{u s}}{V_{u s}},
$$


where $g_{A}$ is the nucleon axial coupling, $g_{A}=1.27$, and $g_{A \Sigma n}$ is the axial coupling of a $\Sigma$ to a neutron, measured to be $0.340 \pm 0.017$ [71]. $D_{n}$ gives a strong bound on $\operatorname{Im} \xi_{u d} / V_{u d}$, at the $10^{-4}$ level. The constraint on $\operatorname{Im} \xi_{u s}$ is at the few-percent level. As we will see, both bounds are significantly weaker than bounds from EDMs and direct CPV in kaon decays.

$c \rightarrow \boldsymbol{d}$ and $c \rightarrow s$ transitions. Analogously to the $u d$ and $u s$ case, we can use the leptonic and semileptonic decays of the $D$ and $D_{s}$ mesons, $D^{+} \rightarrow \mu^{+} \nu_{\mu}, D_{s}^{+} \rightarrow \mu^{+} \nu_{\mu}, D \rightarrow$ $\pi l \nu_{l}$, and $D \rightarrow K l \nu_{l}$, to constrain the vector and axial couplings of a charm quark to $s$ and $d$ quarks. The leptonic decays of the pseudoscalar $D$ and $D_{s}$ mesons probe the axial current, while the semileptonic decays probe the vector current. The experimental input is [71]

$$
\begin{aligned}
f_{D}\left|V_{c d}-\xi_{c d}\right| & =45.91 \pm 1.05 \mathrm{MeV}, \quad f_{D_{s}}\left|V_{c s}-\xi_{c s}\right| & =250.9 \pm 4.0 \mathrm{MeV} \\
f_{+}^{D \pi}(0)\left|V_{c d}+\xi_{c d}\right| & =0.1425 \pm 0.0019, \quad f_{+}^{D K}(0)\left|V_{c s}+\xi_{c s}\right| & =0.728 \pm 0.005
\end{aligned}
$$

and the LQCD input for the $D$ and $D_{s}$ decay constants and form factors is given in table 8 . $\boldsymbol{b} \rightarrow \boldsymbol{u}$ and $\boldsymbol{b} \rightarrow \boldsymbol{c}$ transitions. In the case of $b \rightarrow c$ transitions, the vector component of the charged current is constrained by the semileptonic decay $B \rightarrow D l \nu_{l}$. For the axial component, the purely leptonic decay of the $B_{c}$ meson has not yet been observed. The decay $B \rightarrow D^{*} l \nu_{l}$ depends on both the vector and axial current. In the zero-recoil limit, when $w=v \cdot v^{\prime}=1$, where $v$ and $v^{\prime}$ are the $B$ and $D$ mesons four-velocities, only the axial contribution survives [100]. Using the HFAG averages [101], we can write

$$
\begin{aligned}
\eta_{E W} \mathcal{F}_{D}(1)\left|V_{c b}+\xi_{c b}\right| & =(42.65 \pm 0.72 \pm 1.35) \cdot 10^{-3}, \\
\eta_{E W}^{\prime} \mathcal{F}_{D^{*}}(1)\left|V_{c b}-\xi_{c b}\right| & =(35.81 \pm 0.11 \pm 0.44) \cdot 10^{-3},
\end{aligned}
$$

where $\eta_{E W}=1.012 \pm 0.005$ and $\eta_{E W}^{\prime}=1.015 \pm 0.005$ [71] are electroweak corrections. $\mathcal{F}_{D}(1)$ and $\mathcal{F}_{D^{*}}(1)$ denote the form factors, evaluated at $w=1$, for which we used the FLAG averages in table 8. Angular distributions in $B \rightarrow D^{*} l \nu_{l}$ could provide additional information on the Lorentz structure of the $W b c$ vertex [102].

The inclusive decays $\bar{B} \rightarrow X_{c} l \bar{\nu}_{l}$ also constrain $\xi_{c b}$. Neglecting power corrections of order $\mathcal{O}\left(\Lambda_{\mathrm{QCD}} / m_{b}\right)$, the inclusive semileptonic width into charmed final states is given by

$$
\begin{aligned}
\Gamma\left(B \rightarrow X_{c} l \nu\right)= & \frac{G_{F}^{2} m_{b}^{5}\left|V_{c b}\right|^{2}}{192 \pi^{3}}\left[\left(1+\left|\frac{\xi_{c b}}{V_{c b}}\right|^{2}\right)\left(1-8 \rho+8 \rho^{3}-\rho^{4}-12 \rho^{2} \log \rho\right)\right. \\
& \left.-4 \frac{m_{c}}{m_{b}} \operatorname{Re}\left(\frac{\xi_{c b}}{V_{c b}}\right)\left(1+9 \rho-9 \rho^{2}-\rho^{3}+6 \rho(1+\rho) \log \rho\right)\right]
\end{aligned}
$$

where $\rho=m_{c}^{2} / m_{b}^{2}$. We then set constraints by using the PDG average [71],

$$
\left|V_{c b}^{\mathrm{eff}}\right|=(42.2 \pm 0.8) \cdot 10^{-3} \quad\left(B \rightarrow X_{c} l \nu\right),
$$

where $\left|V_{c b}^{\text {eff }}\right|^{2}=\left|V_{c b}\right|^{2} \Gamma\left(B \rightarrow X_{c} l \nu\right) / \Gamma^{\mathrm{SM}}\left(B \rightarrow X_{c} l \nu\right)$.

The constraints from the inclusive decays we obtain in this way should only be viewed as order-of-magnitude constraints for a number of reasons. First of all, we should take into account power corrections, which are not included in eq. (4.9), in order to obtain $V_{c b}$ 
from inclusive decays [103-106]. Furthermore, eq. (4.10) relies on fits to the leptonic and hadronic moments of the decay distribution. As the dependence on the lepton-energy is not the same for $\xi_{c b}$ and $V_{c b}$ after applying cuts, the SM fit will be altered in the presence of right-handed currents. We should therefore refit the leptonic moments, while taking into account contributions from right-handed currents. Such an analysis is beyond the scope of the current work. We will use eq. (4.10) to estimate the limits from the inclusive measurements, and refer to refs. [107-109] for a more detailed discussion.

In the case of $b \rightarrow u$ transitions, the leptonic channel $B^{+} \rightarrow \tau^{+} \nu_{\tau}$ allows us to determine the axial current $\left|V_{u b}-\xi_{u b}\right|$, while the vector current is probed by $B \rightarrow \pi l \nu_{l}$. Additional exclusive decays, such as $B \rightarrow \rho l \nu_{l}$, can be used to further improve the sensitivity to RHCC [109, 110]. For the leptonic decays, we use the HFAG average of the BaBar and Belle results, $\operatorname{Br}\left(B^{+} \rightarrow \tau \nu\right)=(1.06 \pm 0.19) \cdot 10^{-4}$, and we employ the FLAG extraction for the semileptonic case,

$$
\begin{aligned}
\left|V_{u b}-\xi_{u b}\right| f_{B} & =0.77 \pm 0.07 \\
\left|V_{u b}+\xi_{u b}\right| & =(3.62 \pm 0.14) \cdot 10^{-3}
\end{aligned}
$$

where the decay constant, $f_{B}$, is given in table 8.

The inclusive determination from $B \rightarrow X_{u} l \nu_{l}$ decays suffers from the same problems as the charm-bottom transition. In principle, power corrections should be included [111, 112] and the leptonic spectrum should be refitted taking into account a right-handed current. Since such an analysis is beyond the scope of our work, we take a similar approach as in the case of $V_{c b}$. We thus estimate constraints from inclusive decays by [71],

$$
\sqrt{\left|V_{u b}\right|^{2}+\left|\xi_{u b}\right|^{2}}=\left(4.49 \pm 0.18_{-0.18}^{+0.16}\right) \cdot 10^{-3} \quad\left(B \rightarrow X_{u} l \nu\right) .
$$

Another exclusive determination of $V_{c b}$ and $V_{u b}$ is provided by measurements of $\operatorname{Br}\left(\Lambda_{b}^{0} \rightarrow p \mu^{-} \bar{\nu}\right)_{q^{2}>15 \mathrm{GeV}} / \operatorname{Br}\left(\Lambda_{b}^{0} \rightarrow \Lambda_{c}^{+} \mu^{-} \bar{\nu}\right)_{q^{2}>7 \mathrm{GeV}}$. This ratio of branching fractions can be calculated using lattice determinations of the relevant form factors [113]. Following the procedure outlined in ref. [113] we obtain for the partially integrated decay widths,

$$
\begin{gathered}
\Gamma\left(\Lambda_{b}^{0} \rightarrow p \mu^{-} \bar{\nu}\right)_{q^{2}>15 \mathrm{GeV}}=4.17 \mathrm{ps}^{-1}\left|V_{u b}+\xi_{u b}\right|^{2}+8.17 \mathrm{ps}^{-1}\left|V_{u b}-\xi_{u b}\right|^{2} \pm \sigma_{\text {stat }}^{(p)} \pm \sigma_{\text {syst }}^{(p)}, \\
\Gamma\left(\Lambda_{b}^{0} \rightarrow \Lambda_{c}^{+} \mu^{-} \bar{\nu}\right)_{q^{2}>7 \mathrm{GeV}}=1.41 \mathrm{ps}^{-1}\left|V_{c b}+\xi_{c b}\right|^{2}+6.99 \mathrm{ps}^{-1}\left|V_{c b}-\xi_{c b}\right|^{2} \pm \sigma_{\text {stat }}^{\left(\Lambda_{c}^{+}\right)} \pm \sigma_{\text {syst }}^{\left(\Lambda_{c}^{+}\right)},
\end{gathered}
$$

where the lattice uncertainties are given by

$$
\begin{aligned}
\left(\sigma_{\text {stat }}^{(p)}\right)^{2} & =\left(0.10\left|V_{u b}+\xi_{u b}\right|^{4}+0.33\left|V_{u b}-\xi_{u b}\right|^{4}+0.16\left|V_{u b}^{2}-\xi_{u b}^{2}\right|^{2}\right) \mathrm{ps}^{-2} \\
\left(\sigma_{\text {syst }}^{(p)}\right)^{2} & =\left(0.10\left|V_{u b}+\xi_{u b}\right|^{4}+0.44\left|V_{u b}-\xi_{u b}\right|^{4}+0.050\left|V_{u b}^{2}-\xi_{u b}^{2}\right|^{2}\right) \mathrm{ps}^{-2} \\
\left(\sigma_{\text {stat }}^{\left(\Lambda_{c}^{+}\right)}\right)^{2} & =\left(0.0023\left|V_{c b}+\xi_{c b}\right|^{4}+0.017\left|V_{c b}-\xi_{c b}\right|^{4}+0.0052\left|V_{c b}^{2}-\xi_{c b}^{2}\right|^{2}\right) \mathrm{ps}^{-2} \\
\left(\sigma_{\mathrm{syst}}^{\left(\Lambda_{c}^{+}\right)}\right)^{2} & =\left(0.0053\left|V_{c b}+\xi_{c b}\right|^{4}+0.11\left|V_{c b}-\xi_{c b}\right|^{4}+0.0027\left|V_{c b}^{2}-\xi_{c b}^{2}\right|^{2}\right) \mathrm{ps}^{-2}
\end{aligned}
$$

The ratio of these decay widths is experimentally determined to be [114]

$$
\frac{\operatorname{Br}\left(\Lambda_{b}^{0} \rightarrow p \mu^{-} \bar{\nu}\right)_{q^{2}>15 \mathrm{GeV}}}{\operatorname{Br}\left(\Lambda_{b}^{0} \rightarrow \Lambda_{c}^{+} \mu^{-} \bar{\nu}\right)_{q^{2}>7 \mathrm{GeV}}}=(1.00 \pm 0.04 \pm 0.08) \cdot 10^{-2} .
$$

In principle, measurements of angular distributions and correlations in semileptonic $\Lambda_{b}$ decays could provide more detailed probes of the Lorentz structure of the $W b c$ vertex [115]. 


\begin{tabular}{|c|cccccc|}
\hline & $d_{e}$ & $d_{n}$ & $d_{\mathrm{Hg}}$ & $d_{\mathrm{Xe}}$ & $d_{\mathrm{Ra}}$ & $d_{p, D}$ \\
\hline current limit & $8.7 \cdot 10^{-16}$ & $3.0 \cdot 10^{-13}$ & $6.2 \cdot 10^{-17}$ & $5.5 \cdot 10^{-14}$ & $1.2 \cdot 10^{-10}$ & $\mathrm{x}$ \\
expected limit & $5.0 \cdot 10^{-17}$ & $1.0 \cdot 10^{-15}$ & $6.2 \cdot 10^{-17}$ & $5.0 \cdot 10^{-16}$ & $1.0 \cdot 10^{-14}$ & $1.0 \cdot 10^{-16}$ \\
\hline
\end{tabular}

Table 9. Current limits on the electron [116], neutron [117, 118], mercury [119, 120], xenon [121] and radium $[122,123]$ EDMs in units of $e \mathrm{fm}$ (90\% confidence level). We also show future sensitivities $[124,125]$.

\section{$5 \Delta F=0$ processes: electric dipole moments}

Permanent EDMs of leptons, nucleons, nuclei, atoms, and molecules provide stringent bounds on flavor-diagonal CPV interactions. The right-handed charged-current couplings $\xi_{i j}$ contribute mostly to hadronic and nuclear EDMs. Right-handed couplings of the light quarks, such as $\xi_{u d}$ and $\xi_{u s}$, induce EDMs through the tree-level operators $C_{1,2 L R}^{u d u d}$ and $C_{1,2 L R}^{u s u s}$, while the couplings to heavier quarks, such as $\xi_{u b}$ or $\xi_{t b}$, induce loop corrections to the light quark EDMs, CEDMs, and the Weinberg operator.

In table 9 we summarize the current limits on the EDMs of the electron, nucleons, ${ }^{199} \mathrm{Hg},{ }^{225} \mathrm{Ra}$, and ${ }^{129} \mathrm{Xe}$ that we used in our analysis, as well as projected sensitivities for these systems, and for the EDMs of the proton and deuteron that are targets for storagering experiments.

The calculation of nucleon and nuclear EDMs in terms of the operators in eqs. (3.1) and (3.6) involves two steps. The operators are first matched to an extension of chiral perturbation theory (ChPT) that contains CPV hadronic interactions [126, 127]. The most important interactions are short-range contributions to the nucleon EDM and CPV pionnucleon couplings. The latter give rise to long-range contributions to the nucleon EDM (from pion loops) and, for chiral-symmetry-breaking operators like the quark CEDMs and four-quark operators, dominate the CPV nucleon-nucleon potential. This CPV potential provides the dominant contribution to the EDMs of nuclei and diamagnetic atoms.

The chiral power counting predicts that the four-quark operators $C_{1,2 L R}^{u d u d}$ and $C_{1,2 L R}^{u s u s}$ contribute mainly to the isospin-breaking pion-nucleon coupling $\bar{g}_{1}[11,126]$. $C_{1,2 L R}^{u d u d}$ do not induce the isoscalar coupling $\bar{g}_{0}$, while $C_{1,2 L R}^{u s u s}$ give corrections that, while formally LO, are small with respect to $\bar{g}_{1}$. As discussed in ref. [11], it is possible to calculate the sizes of $\bar{g}_{0,1}$ by noticing that these couplings receive large contributions from tadpole diagrams, which involve the coupling of the neutral pion to the vacuum. The tadpole coupling can be related, at leading order in ChPT, to the $K \rightarrow \pi \pi$ matrix element of the SM electroweak penguin operators $\mathcal{Q}_{7,8}$. Using recent LQCD of these matrix elements [128, 129], it then becomes possible to give a solid estimate of the sizes of $\bar{g}_{0,1}$ [11]. The error on $\bar{g}_{0,1}$ is at the moment dominated by ChPT uncertainties, which we conservatively estimate at the $50 \%$ level.

$\bar{g}_{0,1}$ also receive contributions from the dipole operators $C_{g u}^{u u}, C_{g d}^{d d}$ and $C_{g d}^{s s}$. These contributions can be in principle computed in LQCD [130], but, at the moment, the best estimate comes from QCD sum rules. The sum rules estimates have roughly (50-100)\% uncertainties [131-134]. We thus find that the pion-nucleon couplings induced by the 
operators in eqs. (3.1) and (3.6) are

$$
\begin{aligned}
\frac{\bar{g}_{1}}{2 F_{\pi}}= & -\left((4.5 \pm 2.2)\left(\tilde{C}_{1 L R}^{u s u s}+2 \tilde{C}_{1 L R}^{u d u d}\right)+(22.0 \pm 11.0)\left(\tilde{C}_{2 L R}^{u s u s}+2 \tilde{C}_{2 L R}^{u d u d}\right)\right. \\
& \left.+\left(0.2_{-0.1}^{+0.4}\right)\left(0.7 \tilde{c}_{g u}^{u u}-1.5 \tilde{c}_{g d}^{d d}\right)\right) \times 10^{-6}, \\
\frac{\bar{g}_{0}}{2 F_{\pi}}= & -\left((0.3 \pm 0.1) \tilde{C}_{1 L R}^{u s u s}+(1.3 \pm 0.6) \tilde{C}_{2 L R}^{u s u s}+(0.05 \pm 0.10)\left(0.7 \tilde{c}_{g u}^{u u}+1.5 \tilde{c}_{g d}^{d d}\right)\right) \\
& \times 10^{-6},
\end{aligned}
$$

where $F_{\pi}=f_{\pi} / \sqrt{2}$, all the couplings are evaluated at $\mu=2 \mathrm{GeV}$, and their values in terms of $\xi_{i j}$ can be read off from table 7 . As in table 7 , we have defined $\tilde{c}_{i}=v^{2} \operatorname{Im} C_{i}$. Eqs. (5.1) and (5.2) assume that the strong CP problem is solved by the Peccei-Quinn mechanism [135] which somewhat affects the values of the matrix elements.

A variety of techniques are available for the calculation of the nucleon EDM induced by four-quark and dipole operators in eqs. (3.1) and (3.6). In refs. [11, 136], we estimated the nucleon EDM induced by the four-quark operators in eq. (3.1) by considering long-range contributions induced by the pion-nucleon couplings $\bar{g}_{0,1}$ (see also ref. [137]). This estimate has intrinsically large uncertainties mainly due to uncertainties on $\bar{g}_{0,1}$ and our ignorance of the size of short-range contributions that appear at the same chiral order. Here we use the uncertainty estimate of ref. [11].

The contributions of $\tilde{c}_{\gamma u}^{u u}$ and $\tilde{c}_{\gamma d}^{d d}$, the up- and down-quark EDMs, are known with $\mathcal{O}(15 \%)$ uncertainties [138-140], while the strange contribution is still highly uncertain. While considerable effort is underway for the calculation of the qCEDM contribution to the nucleon EDM [141-143], the best estimate at the moment comes from QCD sum rules, and has an estimated 50\% uncertainty [131-134]. Finally, the Weinberg operator appears with the largest uncertainty, $\mathcal{O}(100 \%)$, based on a combination of QCD sum-rules [144] and naive dimensional analysis estimates [87]. Combining these results, we find

$$
\begin{aligned}
d_{n}= & \left((43 \pm 27) \tilde{C}_{1 L R}^{u s u s}+(210 \pm 130) \tilde{C}_{2 L R}^{u s u s}+(22 \pm 14) \tilde{C}_{1 L R}^{u d u d}+(110 \pm 70) \tilde{C}_{2 L R}^{u d u d}\right. \\
& -(0.93 \pm 0.05) \tilde{c}_{\gamma u}^{u u}-(4.0 \pm 0.2) \tilde{c}_{\gamma d}^{d d}-(0.8 \pm 0.9) \tilde{c}_{\gamma d}^{s s} \\
& \left.-(3.9 \pm 2.0) \tilde{c}_{g u}^{u u}-(16.8 \pm 8.4) \tilde{c}_{g d}^{d d} \pm(320 \pm 260) v^{2} C_{\tilde{G}}\right) \times 10^{-9} e \mathrm{fm} \\
d_{p}= & \left(-(56 \pm 30) \tilde{C}_{1 L R}^{u s u s}-(280 \pm 150) \tilde{C}_{2 L R}^{u s u s}-(42 \pm 26) \tilde{C}_{1 L R}^{u d u d}-(210 \pm 130) \tilde{C}_{2 L R}^{u d u d}\right. \\
& +(3.8 \pm 0.2) \tilde{c}_{\gamma u}^{u u}+(1.0 \pm 0.1) \tilde{c}_{\gamma d}^{d d}-(0.8 \pm 0.9) \tilde{c}_{\gamma d}^{s s)} \\
& \left.+(9.3 \pm 4.6) \tilde{c}_{g u}^{u u}+(9.2 \pm 4.2) \tilde{c}_{g d}^{d d} \mp(320 \pm 260) v^{2} C_{\tilde{G}}\right) \times 10^{-9} e \mathrm{fm} .
\end{aligned}
$$

Finally, using the nuclear calculations of refs. [127, 145-155] we can predict nuclear EDMs in terms of $\bar{g}_{0,1}$ and $d_{n, p}$

$$
\begin{aligned}
d_{D}=(0.94 \pm 0.01)\left(d_{n}+d_{p}\right)-(0.18 \pm 0.02) \frac{\bar{g}_{1}}{2 F_{\pi}} e \mathrm{fm} \\
d_{\mathrm{Hg}}=-(2.8 \pm 0.6) \cdot 10^{-4} \cdot\left[(1.9 \pm 0.1) d_{n}+(0.20 \pm 0.06) d_{p}\right. \\
\left.-\left(0.13_{-0.07}^{+0.5} \frac{\bar{g}_{0}}{2 F_{\pi}}+0.25_{-0.63}^{+0.89} \frac{\bar{g}_{1}}{2 F_{\pi}}\right) \text { e fm }\right]
\end{aligned}
$$




\begin{tabular}{|c|c|c|c|}
\hline $\operatorname{Re} A_{0}$ & $33.201 \cdot 10^{-8} \mathrm{GeV}$ & $\operatorname{Re} A_{2}$ & $1.479 \cdot 10^{-8} \mathrm{GeV}$ \\
$|\epsilon|$ & $(2.228 \pm 0.011) \cdot 10^{-3}$ & $\operatorname{Arg} \epsilon$ & $0.75957 \mathrm{rad}$ \\
$\operatorname{Re}\left(\epsilon^{\prime} / \epsilon\right)$ & $(16.6 \pm 2.3) \cdot 10^{-4}$ & $\operatorname{Br}\left(K^{+} \rightarrow \pi^{0} e^{+} \nu\right)$ & $(5.07 \pm 0.04) \cdot 10^{-2}$ \\
$\tau\left(K_{L}\right)$ & $(5.116 \pm 0.021) \cdot 10^{-8} \mathrm{~s}$ & $\tau\left(K^{+}\right)$ & $(1.2380 \pm 0.0020) \cdot 10^{-8} \mathrm{~s}$ \\
\hline
\end{tabular}

Table 10. Experimental input for the $\Delta S=1$ processes $\epsilon^{\prime} / \epsilon$ and $K_{L} \rightarrow \pi^{0} e^{+} e^{-}[71]$.

$$
\begin{aligned}
& d_{\mathrm{Xe}}=(0.33 \pm 0.05) \cdot 10^{-4} \cdot {\left[(-0.32 \pm 0.02) d_{n}+(0.0061 \pm 0.001) d_{p}\right.} \\
&+\left.\left(0.10_{-0.037}^{+0.53} \frac{\bar{g}_{0}}{2 F_{\pi}}+0.076_{-0.038}^{+0.55} \frac{\bar{g}_{1}}{2 F_{\pi}}\right)\right] e \mathrm{fm} \\
& d_{\mathrm{Ra}}=(7.7 \pm 0.8) \cdot 10^{-4} \cdot\left(-19_{-57}^{+6.4} \frac{\bar{g}_{0}}{2 F_{\pi}}+76_{-25}^{+227} \frac{\bar{g}_{1}}{2 F_{\pi}}\right) e \mathrm{fm}
\end{aligned}
$$

The nucleon EDM contributions to $d_{\text {Ra }}$ have, as far as we know, not been calculated but are expected to be small compared to the large pion-exchange contributions.

The estimates of the nucleon and nuclear EDMs in eqs. (5.3) and (5.4) are affected by large hadronic and nuclear uncertainties. Several matrix elements are consistent with zero and the large uncertainties allow for cancellations between different contributions, which can significantly affect the constraints on the $\xi_{i j}$ couplings. Therefore, when setting constraints, we vary the hadronic and nuclear matrix elements within their allowed ranges in order to minimize the total $\chi^{2}$. This corresponds to the Rfit approach for treating theoretical errors as defined in ref. [156].

\section{$6 \Delta=1$ processes}

In this section we discuss the contribution of RHCC to direct CP violation in kaon decays and to the FCNC decay $K_{L} \rightarrow \pi^{0} e^{+} e^{-}$. While the real parts of the $\xi_{i j}$ elements are well constrained by the leptonic and semileptonic charged-current decays discussed in section 4 , $\epsilon^{\prime} / \epsilon$ and $K_{L} \rightarrow \pi^{0} e^{+} e^{-}$provide additional information on the imaginary parts of the $\xi_{i s}$ and $\xi_{\text {id }}$ elements. $\epsilon^{\prime} / \epsilon$ is dominated by tree-level contributions from the four-quark operators $C_{1,2 L R}^{u d u s}$ and $C_{1,2 L R}^{u s u d}$, while $K_{L} \rightarrow \pi^{0} e^{+} e^{-}$receives correction at one loop. The latter arise from matching the RHCC to the flavor-changing dipole operators $C_{\gamma d}^{d s}$ and $C_{\gamma d}^{s d}$, which are particularly important for internal charm and top quarks. Other $\Delta S=1 \mathrm{FCNC}$ decays, such as $K_{L} \rightarrow \pi^{0} \nu \bar{\nu}$, receive contributions from RHCC that are only quadratic in $\xi$ and therefore play a less important role. We discuss them briefly in appendix A.2. In table 10 we list the experimental input needed in sections 6.1 and 6.2 .

\section{$6.1 \epsilon^{\prime} / \epsilon$}

$\xi_{u d}$ and $\xi_{u s}$ give large contributions to direct $\mathrm{CP}$ violation in $K_{L} \rightarrow \pi \pi$ decays, while indirect $\mathrm{CP}$ violation in kaon mixing is not significantly affected [11]. Direct CP violation 
is quantified by $\epsilon^{\prime}$, which can be expressed as

$$
\operatorname{Re}\left(\frac{\epsilon^{\prime}}{\epsilon}\right)=\operatorname{Re}\left(\frac{i \omega e^{i\left(\delta_{2}-\delta_{0}\right)}}{\sqrt{2} \epsilon}\right)\left[\frac{\operatorname{Im} A_{2}}{\operatorname{Re} A_{2}}-\frac{\operatorname{Im} A_{0}}{\operatorname{Re} A_{0}}\right] .
$$

Here $A_{0,2} e^{i \delta_{0,2}}=\frac{1}{\sqrt{2}}\left\langle(\pi \pi)_{I=0,2}|\mathcal{H}| K\right\rangle$ are the amplitudes for final-state pions with total isospin $I=0,2$, the corresponding strong phases are denoted by $\delta_{0,2}, \mathcal{H}$ is the weak Hamiltonian, and $\omega \equiv \operatorname{Re} A_{2} / \operatorname{Re} A_{0}=0.04454$.

In the SM, $A_{0}$ and $A_{2}$ are sensitive to contributions from charged-current operators, $\mathcal{Q}_{1-2}$, strong penguin operators, $\mathcal{Q}_{3-6}$, and electroweak penguin operators, $\mathcal{Q}_{7-10}$. The values of their NLO Wilson coefficients have been calculated in refs. [85, 157-159], while lattice determinations of the necessary matrix elements are given in refs. [128, 129, 160]. Combining these results with the experimental values in table 10 and lattice determinations of the strong phases, $\delta_{0}=(23.8 \pm 4.9 \pm 1.2)^{\circ}, \delta_{2}=-(11.6 \pm 2.5 \pm 1.2)^{\circ}$, leads to the SM prediction [160]

$$
\operatorname{Re}\left(\frac{\epsilon^{\prime}}{\epsilon}\right)_{\mathrm{SM}}=(1.38 \pm 5.15 \pm 4.59) \cdot 10^{-4} \simeq(1.4 \pm 6.9) \cdot 10^{-4}
$$

where we combined the statistical and systematical errors in quadrature.

As noticed in ref. [11], chiral symmetry relates the contributions to $\epsilon^{\prime} / \epsilon$ of the fourquark tree-level operators induced by $\xi_{u d}$ and $\xi_{u s}$, given in eq. (3.1), to those of the electroweak penguin operators $\mathcal{Q}_{7}$ and $\mathcal{Q}_{8}$. Such a determination in principle still suffers from higher-order, $\mathcal{O}\left(m_{K}^{2}\right)$, corrections. Fortunately, the $I=3 / 2$ parts of the LR operators, $O_{1 L R}^{u d u s}$ and $O_{2 L R}^{u d u s}$, coincide after an isospin decomposition with those of $\mathcal{Q}_{7}$ and $\mathcal{Q}_{8}$, respectively. Isospin symmetry therefore implies a stronger relation between the contributions of the left-right operators to the $I=2$ amplitude and the matrix elements of $\mathcal{Q}_{7,8}[161,162]$. As this relation depends on isospin arguments, it is only subject to $\mathcal{O}\left(\left(m_{d}-m_{u}\right) / \Lambda_{\chi}\right)$ and $\mathcal{O}(\alpha / \pi)$ corrections, expected at the few-percent level. The resulting expression for the $I=2$ amplitude is [11]

$$
\begin{aligned}
\operatorname{Im} A_{2}(\xi)=\frac{1}{6 \sqrt{2}} \operatorname{Im}[ & \left(C_{1 L R}^{u d u s}-C_{1 L R}^{u s u d^{*}}\right)\left\langle(\pi \pi)_{I=2}\left|\mathcal{Q}_{7}\right| K^{0}\right\rangle \\
& \left.+\left(C_{2 L R}^{u d u s}-C_{2 L R}^{u s u d^{*}}\right)\left\langle(\pi \pi)_{I=2}\left|\mathcal{Q}_{8}\right| K^{0}\right\rangle\right],
\end{aligned}
$$

where $[128,129]$

$$
\left\langle(\pi \pi)_{I=2}\left|\mathcal{Q}_{7}\right| K^{0}\right\rangle=(0.36 \pm 0.02) \mathrm{GeV}^{2}, \quad\left\langle(\pi \pi)_{I=2}\left|\mathcal{Q}_{8}\right| K^{0}\right\rangle=(1.6 \pm 0.094) \mathrm{GeV}^{2}
$$

Such a relation does not exist for the $I=0$ amplitude, however, at leading order in ChPT we obtain $A_{0}(\xi)=-2 \sqrt{2} A_{2}(\xi)$. We thus find

$$
\operatorname{Re}\left(\frac{\epsilon^{\prime}}{\epsilon}\right)=\operatorname{Re}\left(\frac{\epsilon^{\prime}}{\epsilon}\right)_{\mathrm{SM}}+\operatorname{Re}\left(\frac{i \omega e^{i\left(\delta_{2}-\delta_{0}\right)}}{\sqrt{2} \epsilon}\right)\left[\frac{\operatorname{Im} A_{2}(\xi)}{\operatorname{Re} A_{2}}-\frac{\operatorname{Im} A_{0}(\xi)}{\operatorname{Re} A_{0}}\right],
$$

where we use the experimental values for $\operatorname{Re} A_{0,2}$. The expression for $A_{0}(\xi)$ might suffer from relatively large $\mathrm{SU}(3)$ corrections. However, it is the $A_{2}(\xi)$ term that constitutes the dominant $\xi$ contribution to $\epsilon^{\prime}$, while the $A_{0}(\xi)$ term is suppressed by $2 \sqrt{2} \omega \simeq 0.1$. We therefore expect eq. (6.5) to be accurate up to the lattice uncertainties in eq. (6.4). 


\section{2 $\quad K_{L} \rightarrow \pi^{0} e^{+} e^{-}$}

In the SM, the decay $K_{L} \rightarrow \pi^{0} e^{+} e^{-}$has a large direct CPV component dominated by the penguin operators $C_{7 V} \bar{s} \gamma^{\mu} d \bar{e} \gamma_{\mu} e$ and $C_{7 A} \bar{s} \gamma^{\mu} d \bar{e} \gamma_{\mu} \gamma_{5} e$ [85]. In addition, there is a CPeven long-distance component dominated by two-photon exchange and an indirect CPV contribution proportional to the mixing parameter $\epsilon_{K}$. Finally, in the presence of righthanded currents, this decay gets contributions from the dipole operators $C_{\gamma d}^{d s}$ and $C_{\gamma d}^{s d}$. Due to the large factors of $m_{t} / m_{s, d}$ and $m_{c} / m_{s, d}$ that appear in the matching coefficients (3.11), this $K_{L}$ decay is particularly sensitive to the imaginary part of the couplings $\xi_{t d}, \xi_{t s}$ and, to a lesser extent, $\xi_{c d}$ and $\xi_{c s}$.

The decay rate can be expressed in terms of the vector and tensor form factors

$$
\begin{aligned}
\left\langle\pi^{0}\left|\bar{s} \gamma^{\mu} d\right| K_{L}\right\rangle & =\frac{1}{\sqrt{2}} f_{+}^{K^{0} \pi^{+}}\left(q^{2}\right)\left(p_{K}^{\mu}+p_{\pi}^{\mu}\right), \\
\left\langle\pi^{0}\left|\bar{s} \sigma^{\mu \nu} d\right| K_{L}\right\rangle & =i f_{T}^{K \pi}\left(q^{2}\right) \frac{\sqrt{2}}{m_{K}+m_{\pi}}\left(p_{\pi}^{\mu} p_{K}^{\nu}-p_{K}^{\mu} p_{\pi}^{\nu}\right),
\end{aligned}
$$

where $f_{+}^{K \pi}$ (see table 8) is related to the vector form factor in $K^{+} \rightarrow \pi^{0} e^{+} \nu$, while $f_{T}^{K \pi}$ has been computed on the lattice. We will use the evaluation of ref. [163], $f_{T}^{K \pi}=0.417 \pm 0.015$, at a renormalization scale $\mu=2 \mathrm{GeV}$.

The RHCC contribution to the branching ratio is determined by the coupling $C_{T}$

$$
C_{T}(\mu)=-\frac{Q_{d}}{4}\left(m_{s} C_{\gamma d}^{d s *}(\mu)+m_{d} C_{\gamma d}^{s d}(\mu)\right),
$$

where the values of the coefficients at $\mu=2 \mathrm{GeV}$ are given in table 6 . The SM contribution is expressed by the functions $\tilde{y}_{7 V}$ and $\tilde{y}_{7 A}$ [85] given in appendix A.2. The $\xi$ operators also contribute to the penguin operators $C_{7 V}$ and $C_{7 A}$, as discussed in appendix A.2, but these contributions are quadratic in $\xi$ and not enhanced by $m_{t, c} / m_{s}$. We therefore do not include them in our analysis.

In terms of $\tilde{y}_{7 V}, \tilde{y}_{7 A}$, and $C_{T}$, the branching ratio becomes

$$
\operatorname{Br}\left(K_{L} \rightarrow \pi^{0} e^{+} e^{-}\right)=\kappa_{e}\left[\left(\operatorname{Im} \lambda_{t} \tilde{y}_{7 V}+\frac{2}{m_{K}+m_{\pi}} \frac{f_{T}^{K \pi}(0)}{f_{+}^{K \pi}(0)} 16 \pi^{2} \operatorname{Im}\left(v^{2} C_{T}\right)\right)^{2}+\operatorname{Im} \lambda_{t}^{2} \tilde{y}_{7 A}^{2}\right],
$$

where $\lambda_{t}=V_{t s}^{*} V_{t d}$. The factor $\kappa_{e}$ is introduced to cancel the SM dependence on the vector form factor $f_{+}^{K \pi}$ by normalizing to the $K^{+} \rightarrow \pi^{0} e^{+} \nu$ decay rate. $\kappa_{e}$ is defined as

$$
\kappa_{e}=\frac{1}{\left|V_{u s}+\xi_{u s}\right|^{2}} \frac{\tau\left(K_{L}\right)}{\tau\left(K^{+}\right)}\left(\frac{\alpha_{\mathrm{em}}}{2 \pi}\right)^{2} \operatorname{Br}\left(K^{+} \rightarrow \pi^{0} e^{+} \nu\right) \sim\left(\frac{0.225}{\left|V_{u s}+\xi_{u s}\right|}\right)^{2} 6 \cdot 10^{-6},
$$

where we used the experimental values in table 10. The expression in eq. (6.8) involves only the direct CPV contributions from the SM. However, since the experimental limit is currently only sensitive to branching ratios that are roughly two orders of magnitude larger than the SM prediction [71],

$$
\mathrm{BR}\left(K_{L} \rightarrow \pi^{0} e^{+} e^{-}\right)<2.8 \cdot 10^{-10} \quad \text { (90\% C.L.) },
$$

we simply use eq. (6.8) to estimate the branching ratio. 


\begin{tabular}{|c|c|c|c|}
\hline $\mathrm{BR}\left(B \rightarrow X_{d} \gamma\right)$ & $(14.1 \pm 5.7) \cdot 10^{-6}$ & $\mathrm{BR}\left(B \rightarrow X_{s} \gamma\right)$ & $(3.32 \pm 0.15) \times 10^{-4}$ \\
$A_{C P}\left(B \rightarrow X_{d+s} \gamma\right)$ & $0.032 \pm 0.034$ & $A_{C P}(B \rightarrow s \gamma)$ & $0.015 \pm 0.02$ \\
& & $S_{K^{*} \gamma}$ & $-0.16 \pm 0.22$ \\
$\Delta m_{d}$ & $(0.5064 \pm 0.0019) \mathrm{ps}^{-1}$ & $\Delta m_{s}$ & $(17.757 \pm 0.021) \mathrm{ps}^{-1}$ \\
$\Delta \Gamma^{(d)}$ & $(-1.3 \pm 6.7) \cdot 10^{-3} \mathrm{ps}^{-1}$ & $\Delta \Gamma^{(s)}$ & $(0.086 \pm 0.006) \mathrm{ps}^{-1}$ \\
$a_{\mathrm{fs}}^{d}$ & $-0.0020 \pm 0.0016$ & $a_{\mathrm{fs}}^{s}$ & $-0.0006 \pm 0.0028$ \\
\hline
\end{tabular}

Table 11. Experimental input for the processes discussed in section 7 [71, 101]. The branching ratios $\mathrm{BR}\left(B \rightarrow X_{d, s} \gamma\right)$ have a cut on the photon energy, $E_{\gamma}>1.6 \mathrm{GeV}$.

\section{$7 \Delta B=1$ and $\Delta B=2$ processes}

$\Delta B=1 \mathrm{FCNC}$ processes such as $B \rightarrow X_{s, d} \gamma$ lead to very strong constraints on RHCC in the top sector. These processes are described by the effective Hamiltonian

$$
\mathcal{H}_{\mathrm{eff}}=-\frac{4 G_{F}}{\sqrt{2}} V_{t b} V_{t q}^{*}\left[C_{7} \mathcal{O}_{7}+C_{7}^{\prime} \mathcal{O}_{7}^{\prime}+C_{8} \mathcal{O}_{8}+C_{8}^{\prime} \mathcal{O}_{8}^{\prime}\right]
$$

with

$$
\mathcal{O}_{7}=\frac{e}{(4 \pi)^{2}} m_{b} \bar{q}_{L} \sigma^{\mu \nu} b_{R} F_{\mu \nu}, \quad \mathcal{O}_{8}=-\frac{g_{s}}{(4 \pi)^{2}} m_{b} \bar{q}_{L} \sigma^{\mu \nu} G_{\mu \nu}^{a} t^{a} b_{R}
$$

$\mathcal{O}_{7,8}^{\prime}$ have analogous definitions with $L \leftrightarrow R$. Relations between the coefficients in eq. (7.1) and the coefficients of the dipole operators in eq. (3.6) are given by

$$
\begin{aligned}
C_{7}\left(m_{W}\right) & =-\frac{4 \pi^{2} Q_{d}}{V_{t b} V_{t q}^{*}} v^{2} C_{\gamma d}^{q b} & C_{7}^{\prime}\left(m_{W}\right) & =-\frac{4 \pi^{2} Q_{d}}{V_{t b} V_{t q}^{*}} \frac{m_{q}}{m_{b}}\left(v^{2} C_{\gamma d}^{b q}\right)^{*}, \\
C_{8}\left(m_{W}\right) & =\frac{4 \pi^{2}}{V_{t b} V_{t q}^{*}} v^{2} C_{g d}^{q b} & C_{8}^{\prime}\left(m_{W}\right) & =\frac{4 \pi^{2}}{V_{t b} V_{t q}^{*}} \frac{m_{q}}{m_{b}}\left(v^{2} C_{g d}^{b q}\right)^{*} .
\end{aligned}
$$

The coefficients at the scales $\mu=\mu_{b}=2 \mathrm{GeV}$ are given in table 6. From eq. (3.11) we see that the contribution of $\xi_{t b}$ to $C_{7,8}$ and of $\xi_{t s}$ and $\xi_{t d}$ to $C_{7,8}^{\prime}$ are enhanced by $m_{t} / m_{b}$ with respect to the $\mathrm{SM}$, and therefore give rise to large effects in the $B \rightarrow X_{s, d} \gamma$ branching ratios. Information on the phases of the $\xi_{t b}$ and $\xi_{t s}$ elements can be gained by studying the $\mathrm{CP}$ asymmetries in inclusive $B \rightarrow X_{d, s} \gamma$ decays, and in the exclusive channel $B \rightarrow K^{* 0} \gamma$. We discuss the $B \rightarrow X_{d, s} \gamma$ branching ratios in section 7.1, and the inclusive and exclusive $\mathrm{CP}$ asymmetries in sections 7.2 and 7.3 , respectively.

$B \rightarrow X_{d, s} \gamma$ is not very sensitive to RHCC in the $W b c$ vertex. In section 7.4 , we therefore study the corrections from RHCC to $B_{q}-\bar{B}_{q}$ mixing with $q=d$, s. While the contributions to the mass differences $\Delta m_{d}$ and $\Delta m_{s}$ are either quadratic in $\xi$, or suppressed by $m_{b} / m_{t}$ with respect to the SM, corrections to the real and imaginary part of the width are more important and lead to constraints on $\operatorname{Im} \xi_{c b}$ that are comparable to those obtained from the tree-level processes discussed in section 4 .

The experimental input used in this section is taken from refs. [71, 101] and is summarized in table 11. 


\subsection{The $B \rightarrow X_{d, s} \gamma$ branching ratio}

For the $B \rightarrow X_{d, s} \gamma$ branching ratios, we employ the expressions derived in ref. [164] rescaled by the SM predictions of refs. [165-167],

$$
\begin{aligned}
\operatorname{BR}\left(B \rightarrow X_{q} \gamma\right)= & r_{q} \frac{\mathcal{N}}{100} \frac{\left|V_{t q}^{*} V_{t b}\right|^{2}}{\left|V_{c b}\right|^{2}+\left|\xi_{c b}\right|^{2}}\left[a+a_{77}\left(\left|R_{7}\right|^{2}+\left|R_{7}^{\prime}\right|^{2}\right)+a_{7}^{r} \operatorname{Re} R_{7}+a_{7}^{i} \operatorname{Im} R_{7}\right. \\
& +a_{88}\left(\left|R_{8}\right|^{2}+\left|R_{8}^{\prime}\right|^{2}\right)+a_{8}^{r} \operatorname{Re} R_{8}+a_{8}^{i} \operatorname{Im} R_{8}+a_{\epsilon \epsilon}\left|\epsilon_{q}\right|^{2}+a_{\epsilon}^{r} \operatorname{Re} \epsilon_{q} \\
& +a_{\epsilon}^{i} \operatorname{Im} \epsilon_{q}+a_{87}^{r} \operatorname{Re}\left(R_{8} R_{7}^{*}+R_{8}^{\prime} R_{7}^{\prime *}\right)+a_{87}^{i} \operatorname{Im}\left(R_{8} R_{7}^{*}+R_{8}^{\prime} R_{7}^{\prime *}\right) \\
& \left.+a_{7 \epsilon}^{r} \operatorname{Re}\left(R_{7} \epsilon_{q}^{*}\right)+a_{7 \epsilon}^{i} \operatorname{Im}\left(R_{7} \epsilon_{q}^{*}\right)+a_{8 \epsilon}^{r} \operatorname{Re}\left(R_{8} \epsilon_{q}^{*}\right)+a_{8 \epsilon}^{i} \operatorname{Im}\left(R_{8} \epsilon_{q}^{*}\right)\right],
\end{aligned}
$$

where $R_{7,8}=\frac{C_{7,8}\left(m_{t}\right)}{C_{7,8}^{\mathrm{SM}}\left(m_{t}\right)}, R_{7,8}^{\prime}=\frac{C_{7,8}^{\prime}\left(m_{t}\right)}{C_{7,8}^{\mathrm{SM}}\left(m_{t}\right)}, C_{7}^{\mathrm{SM}}\left(m_{t}\right)=-0.189$, and $C_{8}^{\mathrm{SM}}\left(m_{t}\right)=-0.095$. Furthermore, $\mathcal{N}=2.567(1 \pm 0.064) \cdot 10^{-3} \cdot r_{q}$ is a factor that rescales the above expression to the SM predictions of refs. [165-167]. It is given by $r_{s}=\frac{3.36}{3.61}$ and $r_{d}=\frac{1.73}{1.38}$. Finally, $\epsilon_{q}=\frac{V_{u q}^{*} V_{u b}}{V_{t q}^{*} V_{t b}}$ and the numerical values of $a_{i j}$ can be found in ref. [164]. In our analysis, we applied the expressions relevant for the following cut on the photon energy $E_{\gamma}>1.6 \mathrm{GeV}$. For $B \rightarrow X_{d} \gamma$ this requires extrapolating the branching ratio quoted in ref. [101], as discussed in ref. [166],

The branching ratios in eq. (7.4) should be compared with the current experimental world averages [71, 101], which we give in table 11. To derive constraints we follow refs. [168, $169]$ and apply the relative uncertainties on the SM predictions $\sigma_{d}=\frac{0.22}{1.73} \operatorname{BR}\left(B \rightarrow X_{d} \gamma\right)$ $\sigma_{s}=\frac{0.23}{3.36} \mathrm{BR}\left(B \rightarrow X_{s} \gamma\right)$. These theoretical uncertainties are then added in quadrature to the experimental ones.

\subsection{The $B \rightarrow X_{d, s} \gamma$ CP asymmetry}

The phase of $\xi_{t b}$ can be probed by the $B \rightarrow X_{s} \gamma$ CP asymmetry. We employ the expression derived in ref. [170],

$$
\begin{aligned}
\frac{A_{C P}(B \rightarrow s \gamma)}{\pi} \equiv & \frac{1}{\pi} \frac{\Gamma\left(\bar{B} \rightarrow X_{s} \gamma\right)-\Gamma\left(B \rightarrow X_{\bar{s}} \gamma\right)}{\Gamma\left(\bar{B} \rightarrow X_{s} \gamma\right)+\Gamma\left(B \rightarrow X_{\bar{s}} \gamma\right)} \\
\approx & {\left[\left(\frac{40}{81}-\frac{40}{9} \frac{\Lambda_{c}}{m_{b}}\right) \frac{\alpha_{s}}{\pi}+\frac{\Lambda_{17}^{c}}{m_{b}}\right] \operatorname{Im} \frac{C_{2}}{C_{7}}-\left(\frac{4 \alpha_{s}}{9 \pi}+4 \pi \alpha_{s} \frac{\Lambda_{78}}{3 m_{b}}\right) \operatorname{Im} \frac{C_{8}}{C_{7}} } \\
& -\left(\frac{\Lambda_{17}^{u}-\Lambda_{17}^{c}}{m_{b}}+\frac{40}{9} \frac{\Lambda_{c}}{m_{b}} \frac{\alpha_{s}}{\pi}\right) \operatorname{Im}\left(\epsilon_{s} \frac{C_{2}}{C_{7}}\right)
\end{aligned}
$$

where $C_{2}$ is the coefficient of the charged-current operator $\mathcal{O}_{1 L L}^{c b c s}, C_{2}=C_{1 L L}^{c b c s} /\left(V_{c b} V_{c s}^{*}\right)$, which, along with $C_{7,8}$, should be evaluated at the factorization scale $\mu_{b} \simeq 2 \mathrm{GeV}$. We employ the following SM values for these coefficients [170],

$$
C_{2}^{\mathrm{SM}}(2 \mathrm{GeV})=1.204, \quad C_{7}^{\mathrm{SM}}(2 \mathrm{GeV})=-0.381, \quad C_{8}^{\mathrm{SM}}(2 \mathrm{GeV})=-0.175 .
$$

In addition, the $\mathrm{CP}$ asymmetry depends on the scale, $\Lambda_{c} \simeq 0.38 \mathrm{GeV}$, and on three hadronic parameters that are estimated to lie in the following ranges [170],

$$
\Lambda_{17}^{u} \in[-0.33,0.525] \mathrm{GeV}, \quad \Lambda_{17}^{c} \in[-0.009,0.011] \mathrm{GeV}, \quad \Lambda_{78} \in[-0.017,0.19] \mathrm{GeV} .
$$


We use the Rfit procedure to deal with these uncertainties [156].

In the case of $B \rightarrow X_{d} \gamma$ decays, instead of the CP asymmetry $A_{C P}\left(B \rightarrow X_{d} \gamma\right)$, the combined asymmetry $A_{C P}\left(B \rightarrow X_{d+s} \gamma\right)$ is measured. This combination can be expressed as [164],

$$
A_{C P}\left(B \rightarrow X_{d+s} \gamma\right)=\frac{A_{C P}\left(B \rightarrow X_{s} \gamma\right)+R_{d s} A_{C P}\left(B \rightarrow X_{d} \gamma\right)}{1+R_{d s}},
$$

with $R_{d s}=\left(\Gamma\left(B \rightarrow X_{d} \gamma\right)+\Gamma\left(\bar{B} \rightarrow X_{d} \gamma\right)\right) /\left(\Gamma\left(B \rightarrow X_{s} \gamma\right)+\Gamma\left(\bar{B} \rightarrow X_{s} \gamma\right)\right)$. Since the branching ratio of $B \rightarrow X_{s} \gamma$ is significantly larger than that of $B \rightarrow X_{d} \gamma, R_{d s}$ is expected to be at the percent level and $A_{C P}\left(B \rightarrow X_{d+s} \gamma\right)$ is therefore mainly sensitive to $A_{C P}(B \rightarrow s \gamma)$. In addition, the experimental precision on the determination of $A_{C P}\left(B \rightarrow X_{d+s} \gamma\right)$ is of the same order as $A_{C P}\left(B \rightarrow X_{s} \gamma\right)$, such that the latter does not provide any additional constraints.

\subsection{The $B \rightarrow K^{* 0} \gamma$ CP asymmetry}

The time-dependent CP asymmetry in the exclusive decay $B \rightarrow K^{* 0} \gamma$ can be described by

$$
\frac{\Gamma\left(\bar{B} \rightarrow \bar{K}^{* 0} \gamma\right)-\Gamma\left(B \rightarrow K^{* 0} \gamma\right)}{\Gamma\left(\bar{B} \rightarrow \bar{K}^{* 0} \gamma\right)+\Gamma\left(B \rightarrow K^{* 0} \gamma\right)}=S_{K^{*} \gamma} \cos \left(\Delta m_{d} t\right)+C_{K^{*} \gamma} \sin \left(\Delta m_{d} t\right)
$$

Here we are interested in the parameter $S_{K^{*} \gamma}$, which is given by

$$
S_{K^{*} \gamma}=2 \frac{\operatorname{Im} \lambda_{K^{*} \gamma}}{1+\left|\lambda_{K^{*} \gamma}\right|^{2}}, \quad \lambda_{K^{*} \gamma}=\frac{q}{p} \frac{A\left(\bar{B} \rightarrow \bar{K}^{* 0} \gamma\right)}{A\left(B \rightarrow K^{* 0} \gamma\right)}
$$

where the ratio $\frac{q}{p}=\frac{V_{t b} V_{t d}^{*}}{V_{t b}^{*} V_{t d}}$ arises from $B_{d}-\bar{B}_{d}$ mixing. At leading order the coefficient $S_{K^{*} \gamma}$ is generated by the electromagnetic dipole operators, $C_{7}$ and $C_{7}^{\prime}$. The dependence on $C_{7}^{\prime}$ is particularly interesting as this Wilson coefficient is induced by right-handed currents, while being suppressed by $m_{s} / m_{b}$ in the SM. In fact, the leading-order expression is [169, 171],

$$
S_{K^{*} \gamma}=\frac{2 \operatorname{Im}\left(\frac{V_{t b} V_{t d}^{*}}{V_{t b}^{*} V_{t d}} \frac{V_{t b} V_{t s}^{*}}{V_{t b}^{*} V_{t s}^{*}} C_{7} C_{7}^{\prime}\right)}{\left|C_{7}\right|^{2}+\left|C_{7}^{\prime}\right|^{2}}
$$

such that $S_{K^{*} \gamma}$ vanishes unless $C_{7}^{\prime}$ is nonzero. As mentioned above, the SM prediction is rather small $[172,173]$

$$
S_{K^{*} \gamma}^{\mathrm{SM}}=(-2.3 \pm 1.6) \cdot 10^{-2} .
$$

The experimental value for $S_{K^{*} \gamma}$ is given in table 11 .

\section{$7.4 B_{q}-\bar{B}_{q}$ mixing}

Right-handed currents can affect $B_{q}-\bar{B}_{q}$ oscillations through insertions of $\xi$ in $\Delta B=2$ box diagrams that govern this mixing. The contributions to the dispersive part of these amplitudes, $M_{12}$, are either quadratic in $\xi_{i j}$ or suppressed with respect to the SM by a factor of the external quark mass, which is at most $m_{b}^{2} / m_{t}^{2}$. We will therefore neglect the contributions to $M_{12}$ which are linear in $\xi$, as well as the dimension-eight effects discussed in appendix A.3. In contrast, the $\xi_{i j}$ contributions to the absorptive part of the mixing 
amplitude are not suppressed with respect to the SM, as both are proportional to $m_{b}^{2}$. The largest contributions come from $\xi_{c b}$ and $\xi_{c q}$, for which we find

$$
\begin{aligned}
\Gamma_{12}^{(q)}(\xi)= & -\frac{G_{F}^{2} m_{b}^{2} m_{B_{q}} f_{B_{q}}^{2}}{\pi} \sqrt{z}\left(\lambda_{c}^{(q) 2}\left(\sqrt{1-4 z}-(1-z)^{2}\right)-\lambda_{c}^{(q)} \lambda_{t}^{(q)}(1-z)^{2}\right) \times \\
& \times\left[\left(\left[\frac{2}{3} B_{1}-\frac{5}{6} B_{2} R\right] \frac{\xi_{c b}}{V_{c b}}+\frac{1}{3} B_{5} R \frac{\xi_{c q}^{*}}{V_{c q}^{*}}\right) \eta_{11 L L} \eta_{11 L R}\right. \\
& \left.+\left(\left[\frac{2}{3} B_{1}+\frac{1}{6} B_{3} R\right] \frac{\xi_{c b}}{V_{c b}}+B_{4} R \frac{\xi_{c q}^{*}}{V_{c q}^{*}}\right)\left(\eta_{11 L L} \eta_{21 L R}+\eta_{21 L L} \eta_{11 L R}+3 \eta_{21 L L} \eta_{21 L R}\right)\right],
\end{aligned}
$$

where $z \equiv m_{c}^{2} / m_{b}^{2}, \lambda_{i}^{(q)}=V_{i b} V_{i q}^{*}$, and $R=m_{B_{q}}^{2} /\left(m_{b}+m_{q}\right)^{2}$. The $B_{i}$ are given in appendix A.3 and represent the bag factors of the $\Delta B=2$ operators in eq. (A.13). Finally, the $\eta$ factors originate from the RG evolution, between $m_{W}$ and $m_{b}$, of the four-fermion operators in eq. (3.1). These factors relate the four-fermion operators at different scales through $C_{i L L(L R)}\left(m_{b}\right)=\eta_{i j L L(L R)} C_{j L L(L R)}\left(m_{W}\right)$, and are determined by eq. (3.4). Explicitly we have

$$
\begin{aligned}
\eta_{11 L L} & =\frac{1}{2}\left(\eta^{6 / 23}+\eta^{-12 / 23}\right), & \eta_{11 L R} & =\eta^{3 / 23}, \\
\eta_{21 L L} & =\frac{1}{2}\left(\eta^{6 / 23}-\eta^{-12 / 23}\right), & \eta_{21 L R} & =\frac{1}{3}\left(\eta^{-24 / 23}-\eta^{3 / 23}\right),
\end{aligned}
$$

where $\eta=\alpha_{s}\left(m_{W}\right) / \alpha_{s}\left(m_{b}\right)$.

The real part of the right-handed contribution to $\Gamma_{12}$ can be constrained by the width difference between the mass eigenstates, whereas the imaginary parts are probed by the measure of CP violation, $a_{\mathrm{fs}}^{q}[174]$,

$$
\Delta \Gamma^{(q)}=4 \frac{\operatorname{Re}\left(\Gamma_{12}^{(q) *} M_{12}^{(q)}\right)}{\Delta m_{B_{q}}}, \quad a_{\mathrm{fs}}^{q}=1-\left|\frac{q}{p}\right|^{2}=\operatorname{Im}\left(\frac{\Gamma_{12}^{(q)}}{M_{12}^{(q)}}\right) .
$$

As mentioned above, the right-handed corrections to $M_{12}$ are small and we neglect them here, while the SM expression for $M_{12}$ can be found in appendix A.3. The SM values for these quantities are given by [175],

$$
\begin{array}{llrl}
\Delta \Gamma_{\mathrm{SM}}^{(d)} & =(2.61 \pm 0.59) \cdot 10^{-3} \mathrm{ps}^{-1}, & & \Delta \Gamma_{\mathrm{SM}}^{(d)}=(0.085 \pm 0.015) \mathrm{ps}^{-1} \\
\left.a_{\mathrm{fs}}^{d}\right|_{\mathrm{SM}}=(-4.7 \pm 0.6) \cdot 10^{-4}, & \left.a_{\mathrm{fs}}^{s}\right|_{\mathrm{SM}}=(2.22 \pm 0.27) \cdot 10^{-5}
\end{array}
$$

while the current experimental determinations are given in table 11.

\section{Single-coupling constraints}

In this section we discuss the constraints on the various right-handed couplings in the case that a single $\xi_{i j}$ element dominates at the scale of new physics. To obtain bounds we construct a $\chi^{2}$ involving the observables described in sections $4-7$. Furthermore, we assume that the CKM matrix is SM-like and apply the Wolfenstein parametrization to write the 
CKM matrix in terms of $A, \lambda, \bar{\rho}$, and $\bar{\eta}$, up to $\mathcal{O}\left(\lambda^{6}\right)$ corrections [176]. ${ }^{3}$ Since the standard extraction of the CKM elements can be modified by the inclusion of right-handed currents, we determine the SM CKM parameters along with the $\xi_{i j}$ from the $\chi^{2}$. Thus, for each $\xi_{i j}$ we simultaneously fit for $A, \lambda, \bar{\rho}$, and $\bar{\eta}$ as well as the real and imaginary parts of $\xi_{i j}$.

Apart from the observables discussed in the sections above, we include $B \rightarrow J / \psi K$, $B_{q}^{0} \rightarrow \mu^{+} \mu^{-}$and the $\Delta F=2$ processes $\epsilon_{K}, \Delta m_{d}$, and $\Delta m_{s}$. As discussed in appendix A.1, A.2 and A.3, these processes do not get large corrections from the RHCC operators. However, we include these observables in our analysis as they provide an important role in determining the SM CKM parameters.

We do not include other non-leptonic $B$ decays, such as $B \rightarrow \pi \pi$. These processes are affected by right-handed currents at tree level, and a reliable estimate of the corrections requires non-perturbative information on the matrix elements of the four-quark operators $C_{1 L R}$ and $C_{2 L R}$, which, at the moment, is not available. As a result, even without taking into account $\xi$ contributions, we find wider ranges for $\bar{\rho}$ and $\bar{\eta}$ compared to ref. [71], but we expect these differences to have small impact on the bounds on $\xi$.

Finally, most of the observables in sections 4-7 involve theory uncertainties, which we treat by adding them in quadrature to the experimental errors. However, there are several cases in which these uncertainties are large and allow for cancellations, notably in $\epsilon^{\prime} / \epsilon, \epsilon_{K}, d_{n}, d_{\mathrm{Hg}}$, and $A_{C P}(b \rightarrow s \gamma)$. In these specific cases, where cancellations between different contributions can significantly affect the constraints, we treat the theoretical errors using the Rfit approach as defined in [156]. We vary the matrix elements within their allowed ranges and apply those values of the matrix elements that minimize the $\chi^{2}$. This procedure leads to conservative constraints as it allows for cancellations between different contributions.

Using the approach described above, we find the following 90\% C.L. constraints on the real and imaginary part of $\xi_{i j}$

$$
\begin{aligned}
& \operatorname{Re} \xi_{i j} \in\left(\begin{array}{l}
{[-7.0,1.6] \cdot 10^{-4}[-2.1,0.05] \cdot 10^{-3}[-1.4,1.3] \cdot 10^{-3}} \\
{[-1.0,0.8] \cdot 10^{-2}[-4.2,0.55] \cdot 10^{-2}[0.1,3.5] \cdot 10^{-3}} \\
{[-1.0,1.0] \cdot 10^{-4}[-2.1,2.5] \cdot 10^{-4}[-1.4,1.2] \cdot 10^{-3}}
\end{array}\right), \\
& \operatorname{Im} \xi_{i j} \in\left(\begin{array}{ccc}
{[0.15,3.4] \cdot 10^{-6}} & {[0.5,7.9] \cdot 10^{-7}} & {[-0.4,0.7] \cdot 10^{-3}} \\
{[-8.5,7.2] \cdot 10^{-6}} & {[-5.7,7.0] \cdot 10^{-3}[-1.5,0.6] \cdot 10^{-2}} \\
{[-4.2,4.2] \cdot 10^{-5}} & {[-2.5,1.9] \cdot 10^{-4}[-2.4,2.3] \cdot 10^{-3}}
\end{array}\right),
\end{aligned}
$$

where we stress that the bounds are obtained turning on one complex $\xi_{i j}$ element at a time.

For the CKM parameters we obtain the $90 \%$ C.L. allowed ranges in case of the $\xi_{u d}$ fit

$$
\lambda \in[0.2232,0.2255], \quad A \in[0.787,0.827], \quad \bar{\rho} \in[0.060,0.20], \quad \bar{\eta} \in[0.33,0.40] .
$$

These values are in agreement with those found in ref. [71], although the constraints found here are generally weaker. As mentioned above, this is to be expected as the fit of ref. [71]

\footnotetext{
${ }^{3}$ The higher-order terms are mainly important for $\epsilon_{K}$, which we employ to constrain the CKM parameters. The imaginary part of the $V_{c s}^{*} V_{c d}$ term only appears after expanding $V$ to $\mathcal{O}\left(\lambda^{5}\right)$.
} 

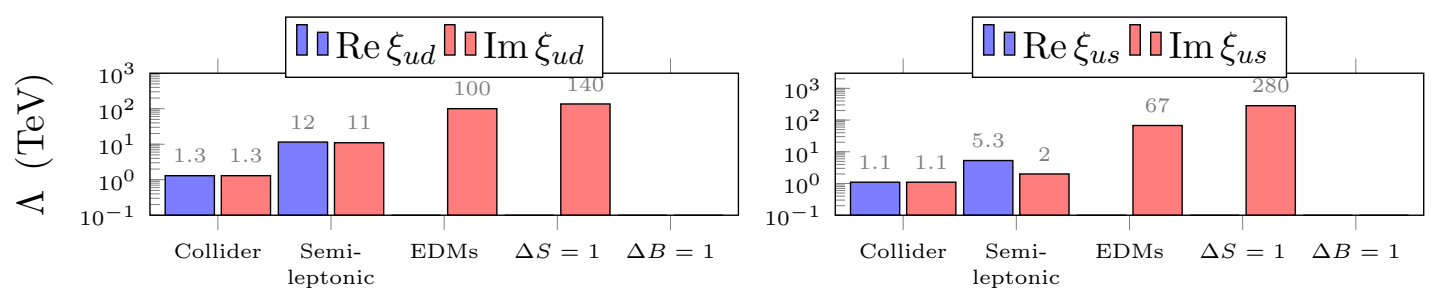

Figure 6. The figure shows naive constraints on the real (in blue) and imaginary (in red) parts of $\xi_{u d}$ and $\xi_{u s}$ converted to an effective scale $\Lambda$ by using $\xi_{i j} \equiv v^{2} / \Lambda^{2}$. These limits are obtained by setting the CKM parameters to the values of ref. [71], and turning on only one real or imaginary part of $\xi_{i j}$ at a time. In the cases where the bounds are asymmetrical, we show the limit that results in the lowest value of $\Lambda$. The different bars represent the limits from the collider, semileptonic, EDM $(\Delta F=0), \Delta S=1$, and $\Delta B=1$ observables, discussed in sections $2,4,5,6$, and 7 , respectively.

includes several more observables than we take into account here. This affects $\bar{\rho}$ the most, which is reflected by the observation that our allowed ranges are roughly twice as wide. In addition, at $68 \%$ C.L. our upper limit for $\bar{\eta}$ extends roughly one standard deviation upwards compared to ref. [71], while our lower limit for $\lambda$ extends one standard deviation downwards. For most of the $\xi_{i j}$ couplings, the ranges in eq. (8.3) are rather stable and do not vary significantly between the different $\xi_{i j}$. The upper and lower ranges of both $\lambda$ and $A$ vary by less than $1 \%$ between the different fits, while $\bar{\rho}$ and $\bar{\eta}$ exhibit variations of up to a few percent. The exception occurs in the case of $\xi_{c b}$, where the upper ranges of $A$ and $\bar{\rho}$ widen by about $5 \%$, while the allowed lower range for $\bar{\eta}$ is widened by roughly $5 \%$.

While the $\chi^{2}$ function used to obtain eqs. (8.1) and (8.2) includes all the observables described in sections $4-7$, the bounds on most entries of the $\xi_{i j}$ elements are dominated by a smaller set of processes. Below we briefly describe which observables drive the constraints for each $\xi$ element.

$\boldsymbol{\xi}_{u d}$ and $\boldsymbol{\xi}_{u s}$. An overview of the constraints on $\operatorname{Re} \xi_{u d}$ is shown in blue in the left panel of figure 6 . These constraints are obtained by setting the CKM parameters to the values of ref. [71] and assuming that only $\operatorname{Re} \xi_{u d}$ is turned on. As these limits indicate, in the single coupling analysis, the best constraints on Re $\xi_{u d}$ come from semileptonic decays, in particular, superallowed $\beta$ decay. Indeed, the unitarity of the CKM matrix $V$ and the absence of modifications of the us element allow one to use leptonic and semileptonic kaon decays to accurately determine $\lambda$, and then extract $\xi_{u d}$ from superallowed $\beta$ decays without relying on leptonic pion decays, which suffer $\sim 1 \%$ percent theoretical uncertainty from the LQCD determination of the pion decay constant. As is shown in red in the left panel of figure $6, \operatorname{Im} \xi_{u d}$ is constrained by the $D$ coefficient, $\epsilon^{\prime} / \epsilon$ and EDMs. The stronger constraint comes from $\epsilon^{\prime} / \epsilon$, followed closely by the neutron EDM. The limit from the $D$ coefficient is two orders of magnitude weaker, $\operatorname{Im} \xi_{u d} \in[-2.9,5.1] \cdot 10^{-4}$. This translates into a difference of one order of magnitude between the semileptonic and EDM constraints on $\Lambda$ in figure 6 .

The situation is very similar for $\xi_{u s}$ as shown the right panel of figure 6 . Here the real part is constrained by leptonic and semileptonic kaon decays, while the imaginary part is constrained by EDMs and $\epsilon^{\prime} / \epsilon$, with the latter giving again the stronger bound. In 

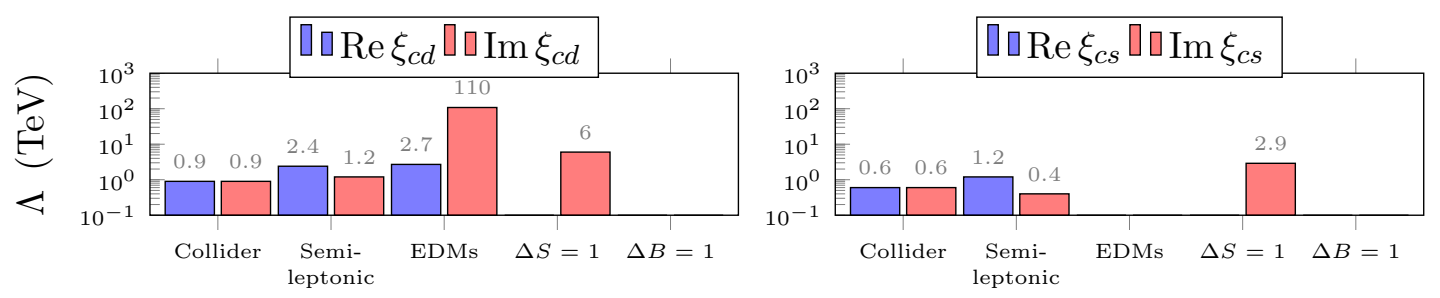

Figure 7. The figure shows naive constraints on $\xi_{c d}$ and $\xi_{c s}$. Notation is the same as in figure 6 .
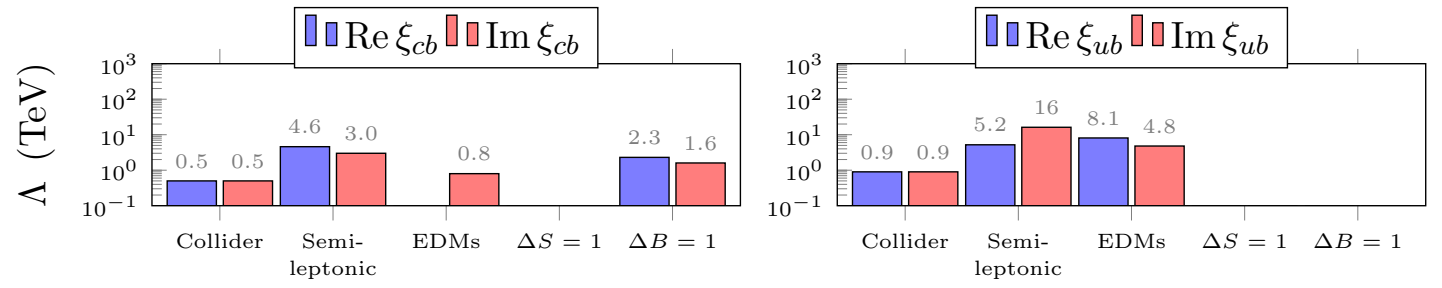

Figure 8. The figure shows naive constraints on $\xi_{c b}$ and $\xi_{u b}$. Notation is the same as in figure 6 .

this case, the semileptonic constraint on the imaginary part is weaker due to the fact that the experimental limit on $D_{\Sigma}$ is significantly less stringent than that on $D_{n}$. As noticed in ref. [11], an imaginary part of $\xi_{u d}$ or $\xi_{u s}$ can solve the $2 \sigma$ discrepancy between the measured value of $\epsilon^{\prime} / \epsilon$ and the SM predictions of refs. [160, 177-180], without conflict with EDM and other low- and high-energy constraints. This manifests in the non-zero values for $\operatorname{Im} \xi_{u d}$ and $\operatorname{Im} \xi_{\text {us }}$ in eq. (8.2).

$\boldsymbol{\xi}_{\boldsymbol{c} d}$ and $\boldsymbol{\xi}_{\boldsymbol{c} s}$. As shown in the left (right) panel of figure 7, the real part of $\xi_{c d}\left(\xi_{c s}\right)$ is mainly constrained by leptonic and semileptonic $D\left(D_{s}\right)$ decays, while the collider limits are weaker by a factor of a few. The larger theoretical and experimental errors cause the bounds from semileptonic decays to be less stringent than for $\xi_{u d}$ and $\xi_{u s}$. The bound on $\operatorname{Im} \xi_{c d}$ is dominated by the neutron EDM, while the small imaginary part of $V_{c d}$ gives rise to a (much weaker) EDM bound on $\operatorname{Re} \xi_{c d}$ as well. $\operatorname{Im} \xi_{c d}$ also contributes to $K_{L} \rightarrow \pi^{0} e^{+} e^{-}$, but the bound is three orders of magnitude weaker, $\left|\operatorname{Im} \xi_{c d}\right|<2 \cdot 10^{-3}$. $\operatorname{Im} \xi_{c s}$ mainly contributes to the nucleon EDM by generating a strange quark EDM. As shown in eq. (5.3), the matrix element linking the neutron EDM to the strange EDM is consistent with zero [138, 139], which, in the Rfit approach, leads to no constraint on $\operatorname{Im} \xi_{c s}$. The bound in eq. (8.2) therefore comes from $K_{L} \rightarrow \pi^{0} e^{+} e^{-}$.

$\boldsymbol{\xi}_{c b}$ and $\boldsymbol{\xi}_{\boldsymbol{u} \boldsymbol{b}}$. As can be seen in figure $8, \xi_{c b}$ and $\xi_{u b}$ are both constrained by the inclusive and exclusive semileptonic $B$ decays. Furthermore, the $B_{q}-\bar{B}_{q}$ oscillation observables, $\Delta \Gamma_{q}$ and $a_{\mathrm{fs}}^{q}$, constrain the real and imaginary parts of $\xi_{c b}$, while EDMs only constrain the imaginary part. Instead, for $\xi_{u b}$ both the real part and imaginary parts are constrained by $d_{n}$ (due to the sizable imaginary part of the relevant CKM element, $V_{u b}$ ), while the contributions to $B_{q}-\bar{B}_{q}$ mixing are negligible.

For both $u b$ and $c b$ elements there is some tension between the determination via inclusive and exclusive decays. We find that adding a right-handed current improves the $\chi^{2}$. 

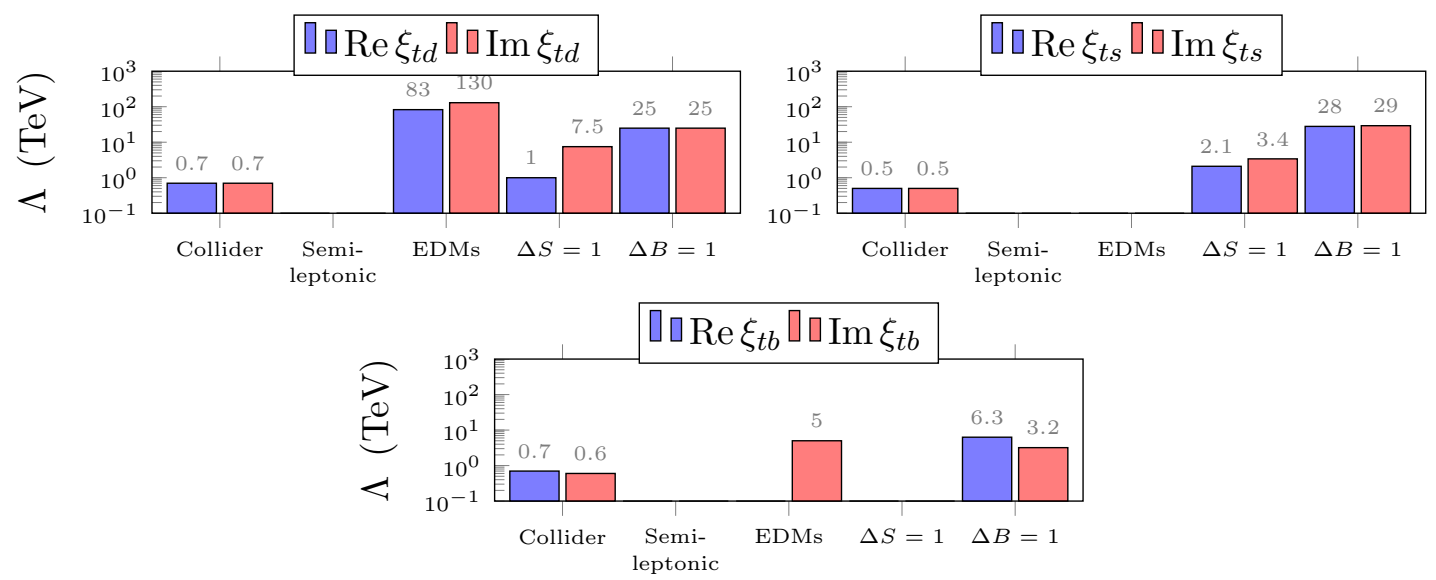

Figure 9. The figure shows naive constraints on $\xi_{t d}, \xi_{t s}$ and $\xi_{t b}$. Notation is the same as in figure 6 .

As shown in eq. (8.1), in the case of $c b$, our fit prefers a non-zero value of Re $\xi_{c b}$, while for the $u b$ element, both real and imaginary part are compatible with zero. The reason being that the nonzero values of $\xi_{u b}$ that are preferred by the semileptonic decays are disfavored by the neutron EDM. We caution though that our analysis of the inclusive decays is incomplete, and, in particular, we did not repeat the fits to the lepton spectrum, which receive different contributions from left- and right-handed currents. We notice that the bounds on $\xi_{u b}$ and $\operatorname{Im} \xi_{c b}$ are rather weak when compared to the magnitudes of $V_{u b}$ and $V_{c b}$, and sizable righthanded corrections (up to $50 \%$ for $V_{u b}$ and $30 \%$ for $V_{c b}$ ) are still allowed.

$\boldsymbol{\xi}_{\boldsymbol{t} \boldsymbol{d}}, \boldsymbol{\xi}_{t s}$, and $\boldsymbol{\xi}_{\boldsymbol{t} \boldsymbol{b}}$. We collect the naive constraints on $\xi_{t d}, \xi_{t s}$, and $\xi_{t b}$ in, respectively, the top-left, top-right, and bottom panels of figure 9. The figure shows that all the top-row elements are strongly constrained by $\Delta B=1$ observables. In particular, $\xi_{t d}$ is constrained by $B \rightarrow X_{d} \gamma$, while for $\xi_{t s, t b}$ stringent limits arise from $B \rightarrow X_{s} \gamma \cdot{ }^{4}$ A comparable limit on $\operatorname{Im} \xi_{t b}$ comes from EDMs, while for $\xi_{t d}$ the EDM limits are stronger than the $\Delta B=1$ constraints by an order of magnitude. Due to the imaginary part of $V_{t d}$, EDMs constrain the real part of $\xi_{t d}$ as well. In contrast, due to the poorly known matrix element related to $\tilde{c}_{\gamma d}^{(s s)}$, there are no EDM constraints on $\xi_{t s}$. Finally, $\xi_{t d}\left(\xi_{t s}\right)$ also contributes to $K_{L} \rightarrow \pi^{0} e^{+} e^{-}$, but the bounds are weaker by roughly a factor 10 (100). For all $\xi_{t j}$ elements the indirect bounds are stronger than the direct collider bounds, by at least an order of magnitude.

\subsection{Summary}

We summarize the strongest constraints on the real and imaginary parts in the left and right panels of figure 10, respectively. The solid bars depict the constraints derived using the Rfit approach for the EDM uncertainties as outlined at the end of section 5. Instead, the dashed bars indicate the 'central' case, in which we set the theory errors in $d_{n}$ and

\footnotetext{
${ }^{4}$ It should be noted that, apart from the allowed range given in eq. (8.1), the flavor and low-energy observables allow for larger negative values of $\operatorname{Re} \xi_{t b}$ namely, $\operatorname{Re} \xi_{t b} \in[-0.034,-0.031]$. However, this possibility is excluded by LHC constraints on $h \rightarrow b \bar{b}$.
} 

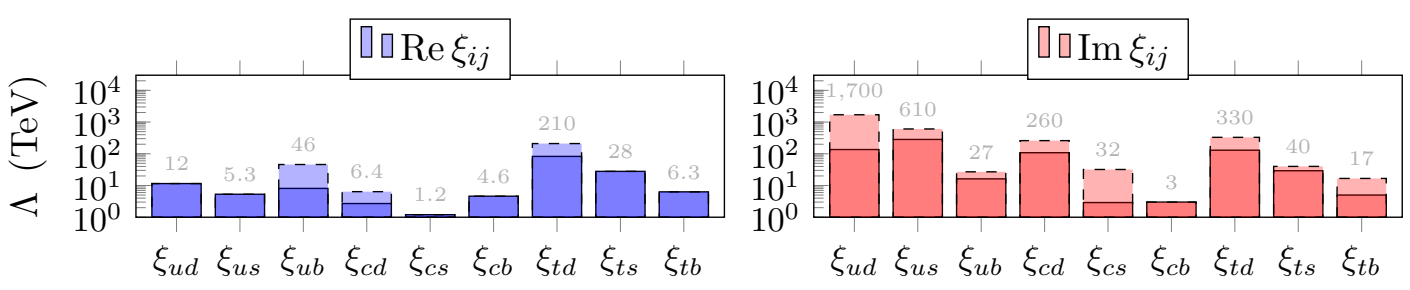

Figure 10. The figure summarizes the most stringent naive constraints on $\xi_{i j}$. The dashed bars show the naive constraints in the case that we do not take into account the theory errors that appear in the EDM expressions eqs. (5.3) and (5.4). Notation is the same as in figure 6.

$d_{\mathrm{Hg}}$ to zero. The difference in the strengths of the constraints illustrates the impact of the hadronic and nuclear uncertainties.

The real parts of $\xi_{t d}$ and $\xi_{t s}$ are the most stringently constrained elements (by $b \rightarrow q \gamma$ ), while the weakest constraints are obtained in the case of $\xi_{c d, c s}$. In the latter case, the precision of the semileptonic decays and the corresponding lattice input is at the percent level, thus allowing for couplings of order $\mathcal{O}\left(10^{-2}\right)$. The remaining real parts are constrained at the sub-percent level. Furthermore, as can be seen from the dashed bars, most of the real parts are unaffected by the theory uncertainties related to EDMs. The exceptions are $\xi_{u b}, \xi_{c d}$, and $\xi_{t d}$, which contribute to EDMs as their corresponding CKM elements have sizable imaginary parts. The main effect of neglecting the theoretical errors is that cancellations in the neutron and mercury EDMs are no longer possible. As a result, the mercury EDM provides the most stringent limit on the real parts of $\xi_{u b, c d, t d}$ in the 'central' case. Although the mercury EDM also constrains $\xi_{t s}$ in the 'central' scenario it does not overtake the $b \rightarrow s \gamma$ limits.

Moving on to the imaginary parts, one can compare the left and right panels of figure 10 to see that, even when using the Rfit approach, the limits on the imaginary parts are generally better than those on the real parts. All constraints are well below the percent level, apart from those on $\xi_{c b}$ and $\xi_{c s}$. The weak bounds on the $c b$ and $c s$ elements result partially due to suppressed contributions to $d_{n}$ : the $\xi_{c s}$ contribution depends on the poorly known strange-EDM matrix element, while the $\xi_{c b}$ contributions only arise at the two-loop level. Among the stronger constraints are those on $\xi_{t d, t s}$ (from $b \rightarrow q \gamma$ and EDMs), however the most impressive limits are set on $\xi_{u d}$ and $\xi_{u s}$ and arise from the neutron EDM and $\epsilon^{\prime} / \epsilon$ which probe effective scales around $\mathcal{O}(100 \mathrm{TeV})$.

As seen from the dashed bars, most constraints on the imaginary parts are at least somewhat affected when moving from the Rfit approach to the 'central' case. For most couplings this results in an improvement of the EDM limit by a factor of a few to $\mathcal{O}(10)$. More drastic changes occur for $\xi_{c s, t s}, \xi_{u d}$, and $\xi_{u s}$. In case of $\xi_{c s}$ this is due to the poorly known strange-EDM matrix element resulting in a vanishing EDM constraint in the Rfit approach, whereas the neutron EDM strongly constraints $\operatorname{Im} \xi_{c s}$ in the central case. The situation is similar for $\xi_{t s}$, although less clear from figure 10 as the improved EDM limits do not overtake the $b \rightarrow s \gamma$ constraints. For $\xi_{u d}$ and $\xi_{u s}$ the EDM limits improve by factors of $\mathcal{O}(300)$ and $\mathcal{O}(100)$, respectively. These large factors arise due to the fact that the 

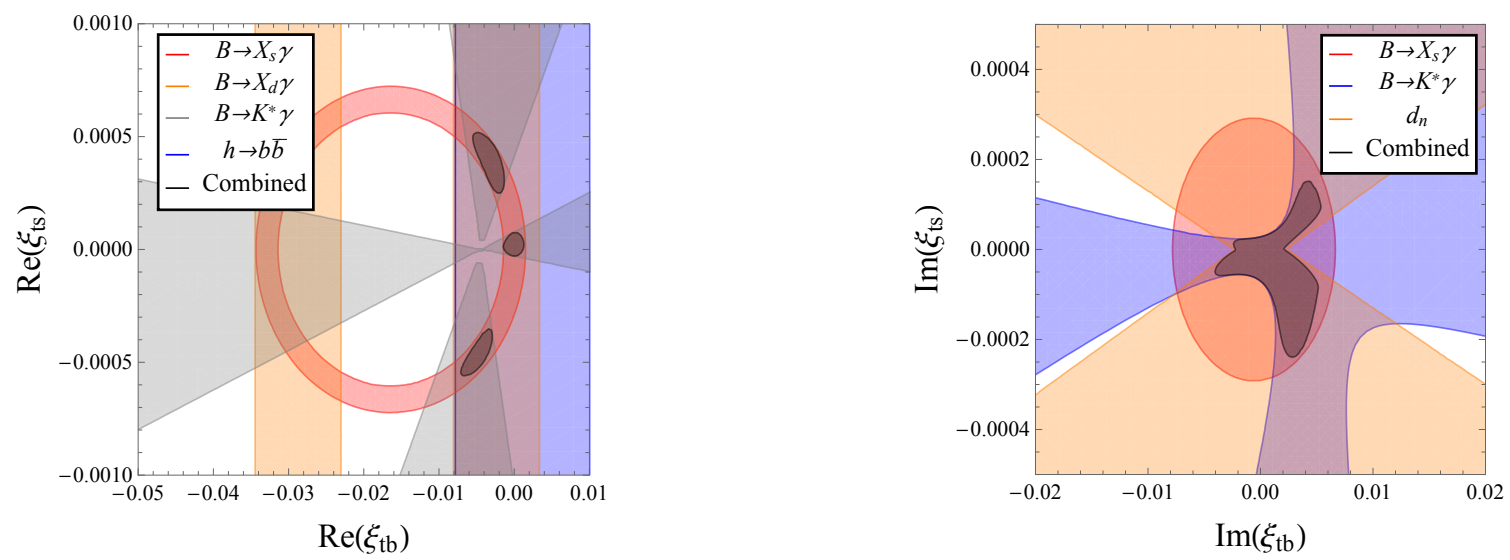

Figure 11. The left (right) panel shows the constraints on the real (imaginary) parts of $\xi_{t b}$ and $\xi_{t s}$. These limits assume that only the real or imaginary parts of $\xi_{t b}$ and $\xi_{t s}$ are generated at the scale $\Lambda$.

four-quark operators $O_{i L R}^{u d u d}$ and $O_{i L R}^{u s u s}$ induce a large pion-nucleon coupling $\bar{g}_{1}$, to which the mercury EDM has an increased sensitivity compared to $d_{n}$. The resulting limits then overtake the $\epsilon^{\prime} / \epsilon$ constraints and naively reach scales up to $\mathcal{O}\left(10^{3}\right) \mathrm{TeV}$.

As the above discussion shows, in some cases the uncertainties related to the matrix elements can mean the difference between a stringent limit or no bound at all. For example, although the neutron and mercury EDMs are in principle sensitive to the right-handed couplings to strange quarks, the poor knowledge of the strange matrix elements does not allow us to set EDM bounds on $\xi_{c s}$ and $\xi_{t s}$. This also holds true for the nuclear matrix elements related to the pion-nucleon couplings $\bar{g}_{0,1}$. These matrix elements allow the mercury EDM to vanish for all $\xi_{i j}$ elements, even though the 'central' limits on $\xi_{u d}$ and $\xi_{u s}$ show that $d_{\mathrm{Hg}}$ could potentially probe scales up to $10^{3} \mathrm{TeV}$. These observations are similar to those discussed in ref. [181] in the context of CPV Higgs-quark interactions, and further motivate studies of hadronic and nuclear matrix elements with lattice QCD and modern nuclear many-body methods.

Finally, it is interesting to see whether the limits given in eq. (8.1) and (8.2) are stable against turning on several $\xi_{i j}$ couplings at the same time. Here we do not commit to a global analysis involving all the $\xi_{i j}$ couplings. Instead, as an example, we briefly discuss the resulting limits when turning on one row of the $\xi_{i j}$ matrix at a time. In this scenario the real parts of the first two rows remain largely unaffected. ${ }^{5}$

The imaginary parts are more sensitive to the effect of turning on additional couplings, as this allows for cancellations in $d_{n}$ and $\epsilon^{\prime} / \epsilon$. As a result, the limit on the imaginary part of $\xi_{u d}$ is now determined by the $D_{n}$ coefficient, giving constraints at the $\mathcal{O}\left(10^{-4}\right)$ level. In turn, this leaves room for an imaginary part of $\xi_{u s}$ up to $\mathcal{O}\left(10^{-3}\right)$. The limit on $\operatorname{Im} \xi_{c d}$ is

\footnotetext{
${ }^{5}$ The only exception is $\xi_{u b}$ for which nonzero values, $\left|\operatorname{Re} \xi_{u b}\right| \approx 2 \cdot 10^{-3}$, are preferred due to the discrepancy between inclusive and exclusive semileptonic decays. Given that our determination of the inclusive decay is not entirely consistent, see section 4, these nonzero values should not be taken too seriously. The reason that $\xi_{u b}$ is consistent with zero in the single-coupling analysis, eq. (8.1), is that nonzero values are disfavored by the neutron EDM. When several couplings can be nonzero at once, however, the neutron EDM limit can be canceled by $\operatorname{Im} \xi_{u d}$ and $\operatorname{Im} \xi_{u s}$ contributions.
} 
weakened by roughly two orders of magnitude due to similar cancellations in $d_{n}$. Instead, the constraints on the imaginary parts of $\xi_{u b, c b}$ and $\xi_{c s}$ do not change much since they are mainly determined by the semileptonic decays and $K_{L} \rightarrow \pi^{0} e^{+} e^{-}$, respectively.

For the third row, the limits on both the real and imaginary parts are weakened by factors of a few for $\xi_{t d, t s}$, while those on $\xi_{t b}$ deteriorate by an order of magnitude. As an example of the interplay between the different $\xi_{t q}$ elements, we show the $\xi_{t b}-\xi_{t s}$ plane for the real (imaginary) parts in the left (right) panel of figure 11. The constraints shown in the left (right) panel assume that only the real (imaginary) parts $\xi_{t b}$ and $\xi_{t s}$ are present at the scale $\Lambda$.

\section{Identifying right-handed currents at low and high energy}

In section 8 we showed that, under the assumption that the SM is modified dominantly by a RHCC at high energy, low-energy bounds from leptonic and semileptonic charged-current decays, $B \rightarrow X_{s, d} \gamma, \epsilon^{\prime} / \epsilon$, and EDMs are significantly stronger than collider bounds. On the other hand, in explicit models of new physics the low-energy observables unavoidably involve some degeneracy [182]. In this section we therefore study more general scenarios in which other operators apart from a RHCC are induced at high energy and how to unambiguously identify RHCCs both at high and low energy. In sections 9.1 and 9.2 we focus on the couplings of the $W$ to $u d$ and us quarks, while in section 9.3 we examine the $W t b$ coupling.

\subsection{Low-energy probes}

In section 8 we used superallowed $\beta$ decays, and leptonic and semileptonic pion and kaon decays to put stringent bounds on RHCC involving the $u$ and $d$, and the $u$ and $s$ quarks. The processes we used to constrain $\operatorname{Re} \xi_{u d}$ and $\operatorname{Re} \xi_{u s}$ are, however, sensitive not only to RHCC, but can be affected by additional contributions. These can be studied by considering the most general semileptonic dimension-six Lagrangian at low energy [183, 184, 186]

$$
\begin{aligned}
\mathcal{L}=-\frac{4 G_{F}}{\sqrt{2}} V_{u d}\left[\left(1+\delta V_{u d}+\left(\varepsilon_{L}\right)_{u d}\right) \bar{u} \gamma^{\mu} P_{L} d \bar{l} \gamma_{\mu} P_{L} \nu+\frac{\xi_{u d}}{V_{u d}} \bar{u} \gamma^{\mu} P_{R} d \bar{l} \gamma_{\mu} P_{L} \nu\right. \\
\left.+\frac{1}{2}\left(\varepsilon_{S}\right)_{u d} \bar{u} d \bar{l} P_{L} \nu-\frac{1}{2}\left(\varepsilon_{P}\right)_{u d} \bar{u} \gamma_{5} d \bar{l} P_{L} \nu+\left(\varepsilon_{T}\right)_{u d} \bar{u} \sigma^{\mu \nu} P_{L} d \bar{l} \sigma_{\mu \nu} P_{L} \nu+\text { h.c. }\right]
\end{aligned}
$$

and analogous contributions for the us couplings. In eq. (9.1), we separated the contribution to left-handed currents coming from corrections to the $W$ couplings to left-handed quark or leptons, $\delta V_{u d}$, from a semileptonic four-fermion operator, $\varepsilon_{L}$. While these operators are degenerate at low energy, they have different manifestations at collider experiments $[183,184,186]$. The operators in eq. (9.1) are in direct correspondence with gauge-invariant operators in the basis of ref. [6], and the mapping is discussed in refs. $[183,184]$. Semileptonic operators arising from vertex corrections, $\delta V_{u d}$ and $\xi_{u d}$, are automatically lepton-flavor universal. For the four-fermion operators, $\varepsilon_{L, P, S, T}$, we assumed the couplings to be diagonal in lepton flavor. 
The operators in eq. (9.1) affect all the observables introduced in section 4, which we used to bound $\xi_{u d}$ and $\xi_{u s}$ (see ref. [187] for a comprehensive analysis). For example, superallowed $\beta$ decays receive corrections from the scalar coupling $\varepsilon_{S}$, which shifts $V_{u d}$ into $[184,186,188]$

$$
\left|V_{u d}\left(0^{+} \rightarrow 0^{+}\right)\right|_{\exp }=\left|V_{u d}\right|\left|1+\delta V_{u d}+\left(\varepsilon_{L}\right)_{u d}+\frac{\xi_{u d}}{V_{u d}}+\frac{g_{S}}{2} c_{0^{+}}^{S}(Z)\left(\varepsilon_{S}\right)_{u d}\right|,
$$

where $g_{S}$ is the nucleon matrix element of the scalar current, and $c_{0^{+}}^{S}(Z)$ is a function which depends on the individual nuclear transition. The expression for $\pi^{ \pm} \rightarrow \mu^{ \pm} \nu_{\mu}$ is modified into

$$
\left|V_{u d}(\pi \rightarrow \mu \nu) f_{\pi}\right|_{\exp }=\left|V_{u d}\right|\left|1+\delta V_{u d}+\left(\varepsilon_{L}\right)_{u d}-\frac{\xi_{u d}}{V_{u d}}-\left(\varepsilon_{P}\right)_{u d} \frac{m_{\pi}^{2}}{m_{\mu}\left(m_{d}+m_{u}\right)}\right| f_{\pi},
$$

and, similarly, the ratio of pion and kaon decays

$$
\left(\left|\frac{V_{u s}}{V_{u d}}\right| \frac{f_{K}}{f_{\pi}}\right)_{\exp }=\frac{\left|1+\delta V_{u s}+\left(\varepsilon_{L}\right)_{u s}-\frac{\xi_{u s}}{V_{u s}}-\left(\varepsilon_{P}\right)_{u s} \frac{m_{K}^{2}}{m_{\mu}\left(m_{s}+m_{u}\right)}\right|\left|V_{u s}\right| f_{K}}{\left|1+\delta V_{u d}+\left(\varepsilon_{L}\right)_{u d}-\frac{\xi_{u d}}{V_{u d}}-\left(\varepsilon_{P}\right)_{u d} \frac{m_{\pi}^{2}}{m_{\mu}\left(m_{d}+m_{u}\right)}\right|\left|V_{u d}\right| f_{\pi}} .
$$

Analogously, the semileptonic decay $K^{0} \rightarrow \pi^{+} l \nu_{l}$ receives contributions from $\delta V_{u s},\left(\varepsilon_{S}\right)_{u s}$, and $\left(\varepsilon_{T}\right)_{u s}$.

The difficulty in identifying a right-handed current at low energies can be illustrated by looking at the degeneracy with anomalous left-handed currents. By setting the fourfermion couplings to zero but allowing for nonzero values of $\delta V_{u d}$ and $\delta V_{u s}$, we obtain significantly weaker constraints on, for example, $\xi_{u d}$

$$
\operatorname{Re} \xi_{u d} \in[-1.0,0.7] \cdot 10^{-2}
$$

which is in reach of future collider searches. The bound is determined by the theoretical uncertainty of $f_{\pi}$, which is at the percent level [93]. If we only allow BSM effects in the left- and right-handed currents, $\xi_{u d}$ and $\delta V_{u d}$ are completely anticorrelated, since the vector combination $\left|V_{u d}+\xi_{u d}\right|$ has to satisfy the stringent constraints from superallowed $\beta$ decays. Introducing additional operators further weakens the bounds on $\xi_{u d}$ and $\xi_{u s}$ [187]. Still, in order to not disrupt the agreement between the SM and the data for leptonic and semileptonic decays, strong correlations between the operators in eq. (9.1) must exist, posing non-trivial constraints on models of new physics.

In light of the intrinsic degeneracy of the observables used in section 4, one might ask if there is a more direct way to access RHCCs at low energy. Decay correlations in the neutron and hyperon $\beta$ decays are particularly sensitive to the Lorentz structure of the quark and lepton coupling [184, 186-189]. For example, the $\beta$ and neutrino asymmetry in neutron $\beta$ decay can be expressed as $[184,186]$

$$
A\left(E_{e}\right)=\frac{2 \lambda(1-\lambda)}{1+3 \lambda^{2}}, \quad B\left(E_{e}\right)=\frac{2 \lambda(1+\lambda)}{1+3 \lambda^{2}}
$$




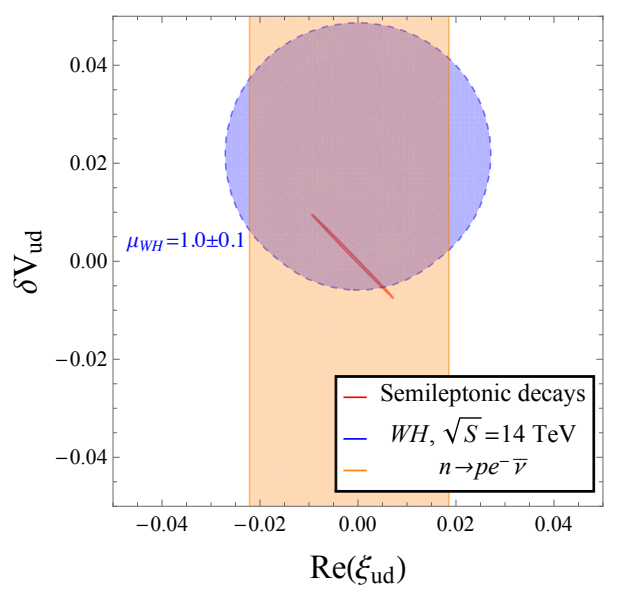

Figure 12. The figure shows the constraints in the $\xi_{u d}-\delta V_{u d}$ plane, after marginalizing over $\xi_{u s}$ and $\delta V_{u s}$. The blue line depicts the constraint from $W H$ production, while the red line indicates the limits from superallowed $\beta$ decay and leptonic pion decay. The vertical orange band results from the experimental determination of $\lambda$, from neutron decay correlations, in combination with an assumed lattice determination of $g_{A}=1.27 \pm 0.05$.

In the presence of the most general modification of the semi-leptonic dimension-six Lagrangian $[188,190]$, we have

$$
\lambda=\frac{g_{A}}{g_{V}}\left|\frac{1+\delta V_{u d}+\left(\varepsilon_{L}\right)_{u d}-\frac{\xi_{u d}}{V_{u d}}}{1+\delta V_{u d}+\left(\varepsilon_{L}\right)_{u d}+\frac{\xi_{u d}}{V_{u d}}}\right|=\frac{g_{A}}{g_{V}}\left(1-2 \operatorname{Re}\left(\frac{\xi_{u d}}{V_{u d}}\right)\right)+\mathcal{O}\left(\frac{v^{4}}{\Lambda^{4}}\right),
$$

where $g_{V}$ and $g_{A}$ are the nucleon matrix elements of the vector and axial-vector currents. Experimentally, the ratio $g_{A} / g_{V}$ is determined with per mil uncertainties, $\lambda=1.2723 \pm$ 0.0023. In order to constrain $\xi_{u d}$ one needs precise information on $g_{A}$. While this is the subject of intense research in LQCD, current determinations of $g_{A}$ have about a $4-5 \%$ uncertainty [140, 185], which allows for percent-level right-handed contributions, as first discussed in ref. [187]. Setting the central value of $g_{A}$ to 1.27 , and assigning a $4 \%$ theoretical error, $g_{A}=1.27 \pm 0.05$, would result in $\operatorname{Re} \xi_{u d} \in[-2.1,2.0] \cdot 10^{-2}$, in the same range as the values probed by $W H$ production. To illustrate the interplay between the different lowenergy and collider constraints, we show in figure 12 the limits in the $\xi_{u d}-\delta V_{u d}$ plane, after marginalizing over $\xi_{u s}$ and $\delta V_{u s}$. As can be seen from the figure, superallowed $\beta$ decay and $\pi \rightarrow \mu \nu$ currently provide the strongest limits. However, as mentioned, these observables get additional contributions from scalar and pseudo-scalar interactions $\left(\varepsilon_{S}\right.$ and $\left.\varepsilon_{P}\right)$, and do not uniquely probe RHCCs. The experimental determination of $\lambda$ combined with lattice calculation of $g_{A}$ provides a direct low-energy probe of right-handed currents in the $u d$ sector. Currently this leads to a constraint that is comparable to future collider limits.

\subsection{Collider probes}

In similar fashion we can ask whether a discrepancy in a collider setting could be unambiguously attributed to a RHCC. In this section we focus on observables related to $W H$ productions as this process, as discussed in section 2.2 , is particular sensitive to 

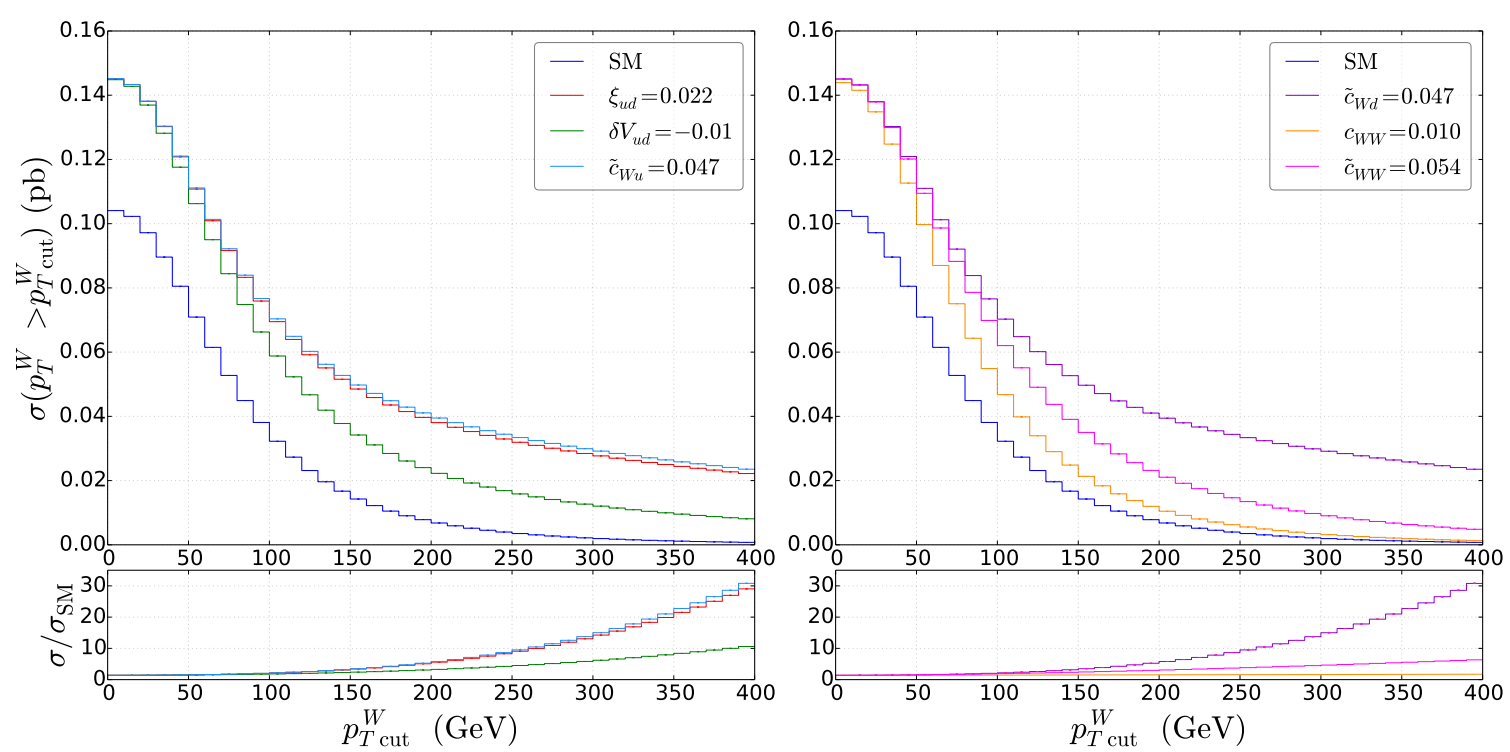

Figure 13. $W^{+} H$ cross section for $p_{T}^{W}>p_{T \text { cut }}^{W}$, at $\sqrt{S}=14 \mathrm{TeV}$. The blue line denotes the SM cross section, the remaining lines include the contributions of the operators in eq. (9.10).

right-handed interactions. To address the issue of identifying the $\xi$ operator, we explore observables that could disentangle RHCCs from other BSM contributions. We consider the full set of dimension-six operators that modifies $W H$ production using the basis of ref. [6]

$$
\begin{aligned}
\mathcal{L}_{6}= & C_{\varphi W} \varphi^{\dagger} \varphi W_{\mu \nu} W^{\mu \nu}+C_{\varphi \tilde{W}} \varphi^{\dagger} \varphi \tilde{W}_{\mu \nu} W^{\mu \nu} \\
& +\varphi^{\dagger} \tau^{I} i \overleftrightarrow{D}_{\mu} \varphi \bar{q}_{L} \tau^{I} \gamma^{\mu} c_{Q \varphi}^{(3)} q_{L}+\varphi^{\dagger} i \overleftrightarrow{D}_{\mu} \varphi \bar{q}_{L} \gamma^{\mu} c_{Q \varphi}^{(1)} q_{L} \\
& -\frac{g}{\sqrt{2}} \bar{q}_{L} \sigma^{\mu \nu} \Gamma_{W}^{u} \tau^{I} W_{\mu \nu}^{I} \tilde{\varphi} u_{R}-\frac{g}{\sqrt{2}} \bar{q}_{L} \sigma^{\mu \nu} \Gamma_{W}^{d} \tau^{I} W_{\mu \nu}^{I} \varphi d_{R} \\
& +\frac{2}{v^{2}} i \tilde{\varphi}^{\dagger} D_{\mu} \varphi \bar{u}_{R} \gamma^{\mu} \xi d_{R}+\text { h.c. },
\end{aligned}
$$

where $\varphi$ is the Higgs doublet, $\tau^{I}$ are Pauli matrices, $\tilde{\varphi}=i \tau_{2} \varphi^{*}, W_{\mu \nu}^{I}$ denotes the $\operatorname{SU}(2)$ field strengths, and $\tilde{W}^{\mu \nu}=\varepsilon^{\mu \nu \alpha \beta} W_{\alpha \beta} / 2$. The Higgs covariant derivatives are given by

$$
\varphi^{\dagger} i \overleftrightarrow{D}_{\mu} \varphi=i \varphi^{\dagger}\left(D_{\mu}-\overleftarrow{D}_{\mu}\right) \varphi, \quad \varphi^{\dagger} \tau^{I} i \overleftrightarrow{D}_{\mu} \varphi=i \varphi^{\dagger}\left(\tau^{I} D_{\mu}-\overleftarrow{D}_{\mu} \tau^{I}\right) \varphi
$$

For fermionic operators we consider couplings to the $u$ and $d$ quarks (the us couplings can be studied in analogous fashion), which, in the mass basis, can be written as

$$
\begin{aligned}
\mathcal{L}= & \frac{h}{v}\left(c_{W W} W^{\mu \nu} W_{\mu \nu}+\tilde{c}_{W W} \tilde{W}^{\mu \nu} W_{\mu \nu}\right)+\frac{g}{\sqrt{2}} V_{u d}\left(1+\delta V_{u d}\left(1+\frac{h}{v}\right)^{2}\right) \bar{u}_{L} \gamma^{\mu} d_{L} W_{\mu}^{+} \\
& +\frac{g}{\sqrt{2}} \xi_{u d}\left(1+\frac{h}{v}\right)^{2} \bar{u}_{R} \gamma^{\mu} d_{R} W_{\mu}^{+} \\
& -\frac{g V_{u d}}{\sqrt{2} v}\left(1+\frac{h}{v}\right)\left(c_{W d} \bar{u}_{L} \sigma^{\mu \nu} d_{R}+c_{W u} \bar{u}_{R} \sigma^{\mu \nu} d_{L}\right) W_{\mu \nu}^{+}+\text {h.c. }
\end{aligned}
$$

The CKM factors in the previous expressions arise from rotating to the mass basis. In order to avoid flavor-changing neutral currents at tree level, we impose that the matrices 

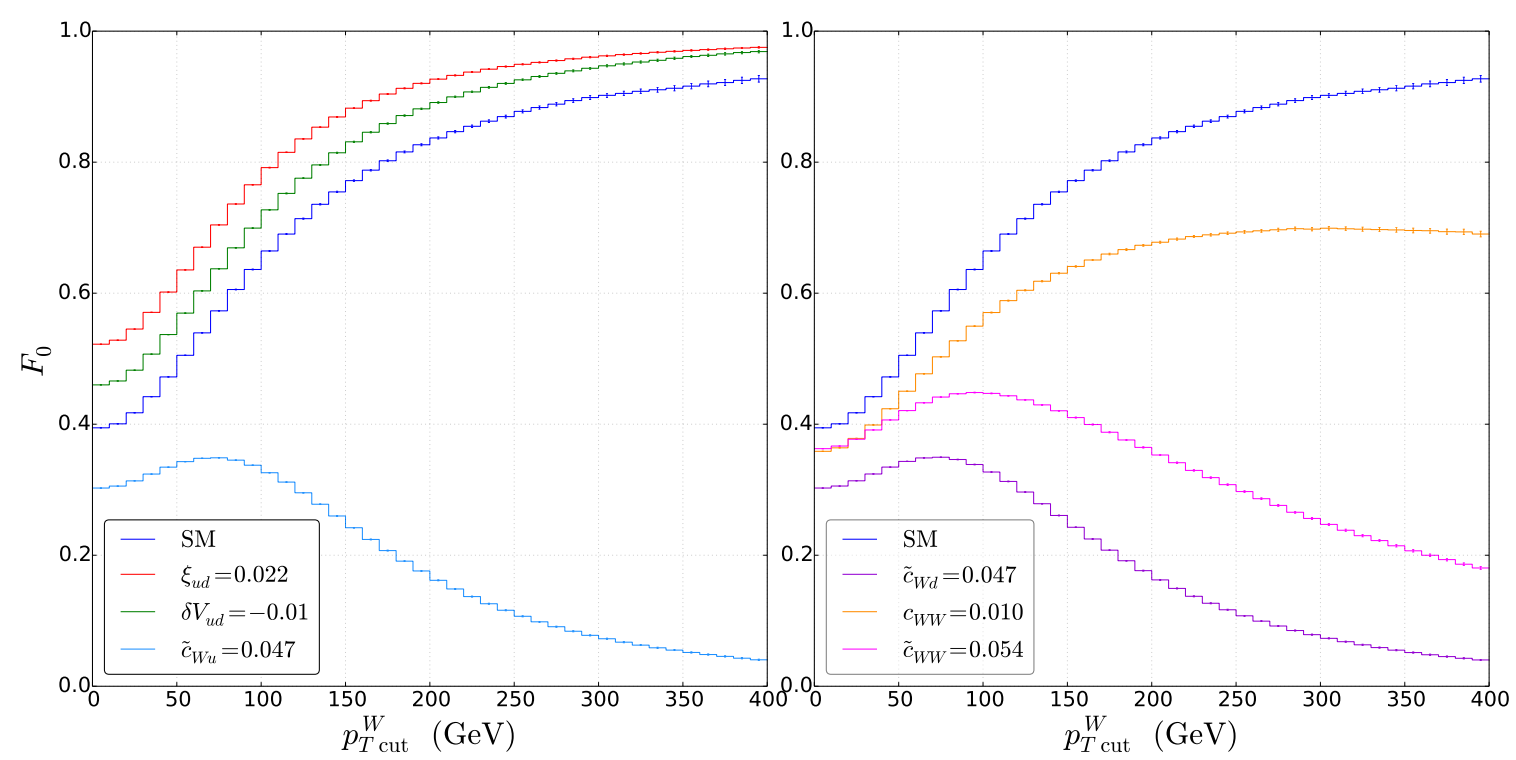

Figure 14. Longitudinal polarization of the $W^{+}$boson in $W^{+} H$ production as a function of $p_{T \text { cut }}^{W}$, at $\sqrt{S}=14 \mathrm{TeV}$.
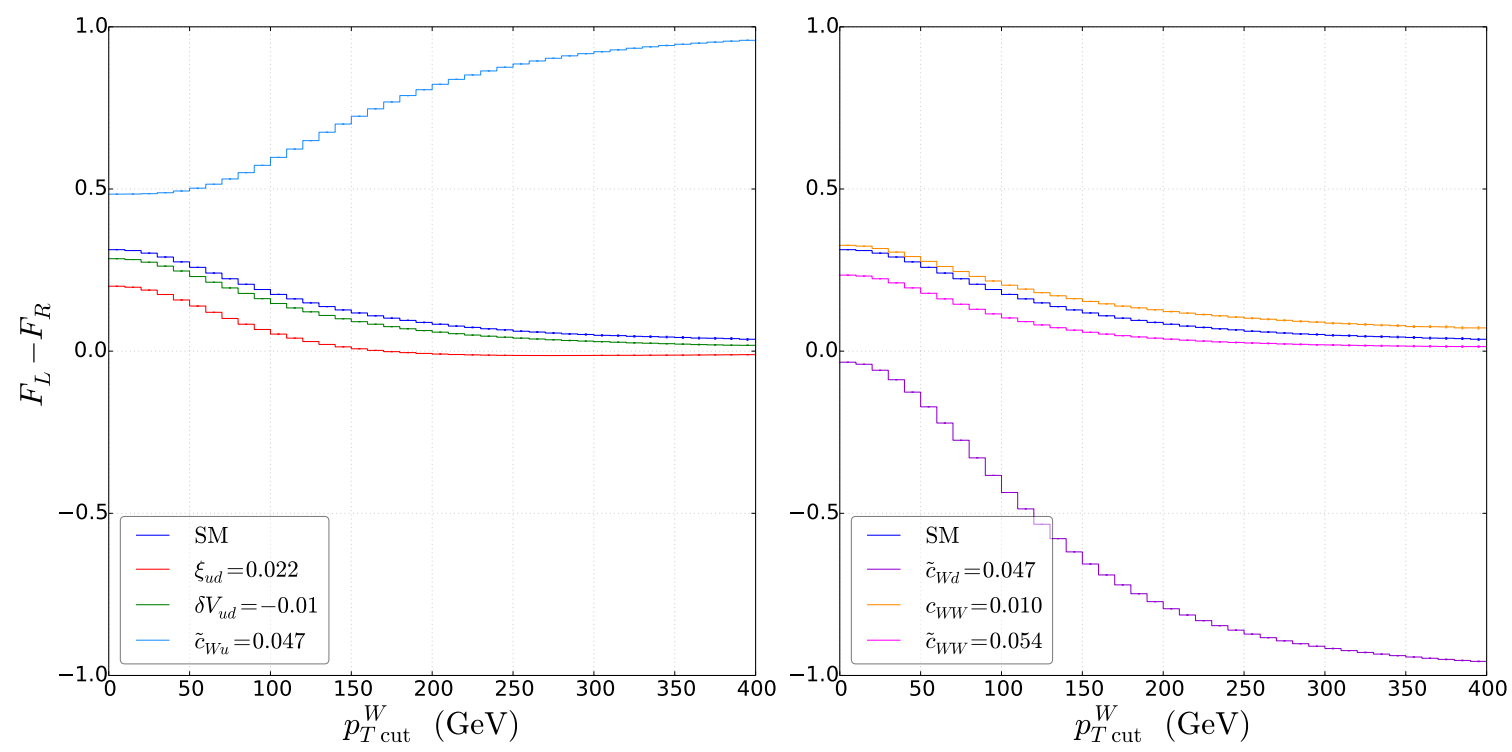

Figure 15. Difference of left and right-handed polarizations of the $W^{+}$boson in $W^{+} H$ production as a function of $p_{T \text { cut }}^{W}$, at $\sqrt{S}=14 \mathrm{TeV}$.

$\Gamma_{W}^{u}, \Gamma_{W}^{d}, c_{Q \varphi}^{(1)}$, and $c_{Q \varphi}^{(3)}$ are diagonal in the mass basis. In addition to $\xi_{u d}$ we then need to consider the dimensionless couplings

$$
\begin{aligned}
c_{W W} & =v^{2} C_{\varphi W}, & \tilde{c}_{W W} & =v^{2} C_{\varphi \tilde{W}}, \\
\delta V_{u d} & =\left(v^{2} c_{Q \varphi}^{(3)}\right)_{11}, & \tilde{c}_{W d} & =\left(v^{2} \Gamma_{W}^{d}\right)_{11},
\end{aligned}
$$

These couplings scale as $v^{2} / \Lambda^{2}$. Hermiticity implies that $c_{W W}, \tilde{c}_{W W}$, and $\delta V_{u d}$ are real, whereas $\xi_{u d}, \tilde{c}_{W u}$, and $\tilde{c}_{W d}$ in general have real and imaginary parts. Since we are neglecting 


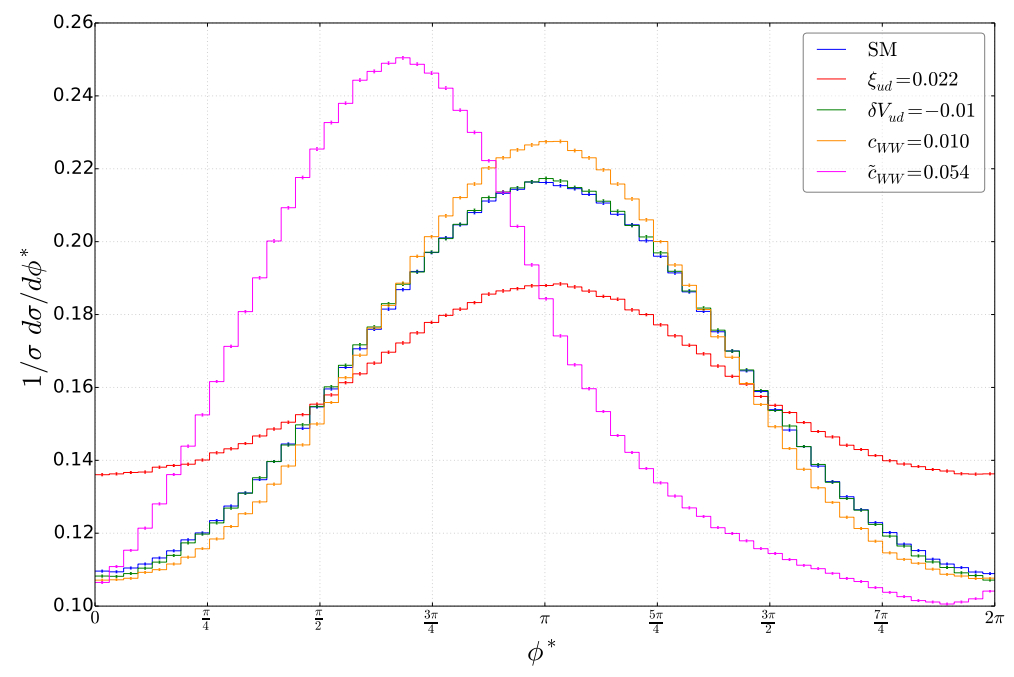

Figure 16. $W^{+} H$ differential cross section with respect to $\phi^{*}$ at $\sqrt{S}=14 \mathrm{TeV}$, in the SM (blue line), and in the presence of dimension-six operators.

interference terms proportional to the light quark masses, the cross section only depends on the absolute values of these couplings.

To illustrate the diagnostic power of collider measurements, we set $\xi_{u d}=0.022$. This value is still allowed by the 8 and $13 \mathrm{TeV}$ data and produces a $40 \%$ modification of the signal strength at $14 \mathrm{TeV}$. We then proceed by turning on one of the couplings in eq. (9.10) at a time, and tune them such that they give the same signal-strength modification as $\xi_{u d}$. In figure 13 we show the effect of the different couplings on the cumulative $W^{+} H$ cross section for $p_{T}^{W}>p_{T \text { cut }}^{W}$, where $p_{T}^{W}$ is the transverse momentum of the $W$ boson. In general this observable receives different corrections from different operators. However, as can be seen from the figure, this observable is not sufficient to lift the degeneracy. In particular, $\xi_{u d}$ and the dipole operators, $\tilde{c}_{W u}$ and $\tilde{c}_{W d}$, induce very similar corrections.

A more suitable observable to disentangle the effects of the various BSM contributions is the angular distributions of the charged lepton coming from the decay of the $W$ boson. We work in the $W$-boson rest frame, with the direction of the $z$-axis along the momentum of the $W$ boson in the lab frame. $\theta^{*}$ is the polar angle of the charged lepton in this frame. The $x$-axis is in the direction orthogonal to the Higgs and $W$ momenta $\hat{x} \sim\left(\vec{p}_{W} \times \vec{p}_{H}\right)$. In this frame, we define the azimuthal angle $\phi^{*}$ as the angle between the plane containing the $W$ and the Higgs bosons, and the plane containing the $W$ and its charged decay product. That is

$$
\cos \phi^{*}=\frac{\left(\vec{p}_{W} \times \vec{p}_{H}\right) \cdot\left(\vec{p}_{W} \times \vec{p}_{e}\right)}{\left|\vec{p}_{W} \times \vec{p}_{H}\right|\left|\vec{p}_{W} \times \vec{p}_{e}\right|}
$$

and we note that $\phi^{*}$ is invariant under boosts along the $W$ momentum $\vec{p}_{W}$.

The angular distribution of the $W$ boson in this frame is parameterized by 8 coefficients, which completely characterize the $W$-boson spin-density matrix

$$
\frac{1}{\sigma} \frac{d \sigma}{d \cos \theta^{*} d \phi^{*}}=\frac{3}{16 \pi}\left[1+\cos ^{2} \theta^{*}+\frac{A_{0}}{2}\left(1-3 \cos ^{2} \theta^{*}\right)\right.
$$




$$
\begin{aligned}
& +A_{1} \sin 2 \theta^{*} \cos \phi^{*}+\frac{A_{2}}{2} \sin ^{2} \theta^{*} \cos 2 \phi^{*} \\
& +A_{3} \sin \theta^{*} \cos \phi^{*}+A_{4} \cos \theta^{*}+A_{5} \sin \theta^{*} \sin \phi^{*} \\
& \left.+A_{6} \sin 2 \theta^{*} \sin \phi^{*}+A_{7} \sin ^{2} \theta^{*} \sin 2 \phi^{*}\right] .
\end{aligned}
$$

The differential distributions with respect to $\theta^{*}$ and $\phi^{*}$ are obtained by integrating eq. (9.13), and are given by

$$
\begin{aligned}
\frac{1}{\sigma} \frac{d \sigma}{d \cos \theta^{*}} & =\frac{3}{8}\left[1+\cos ^{2} \theta^{*}+\frac{A_{0}}{2}\left(1-3 \cos ^{2} \theta^{*}\right)+A_{4} \cos \theta^{*}\right], \\
\frac{1}{\sigma} \frac{d \sigma}{d \phi^{*}} & =\frac{1}{2 \pi}\left[1+\frac{3 \pi}{16}\left(A_{3} \cos \phi^{*}+A_{5} \sin \phi^{*}\right)+\frac{1}{4}\left(A_{2} \cos 2 \phi^{*}+A_{7} \sin 2 \phi^{*}\right)\right] .
\end{aligned}
$$

The coefficients $A_{0}$ and $A_{4}$ are related to the $W$-boson helicity fractions [35],

$$
F_{0}=\frac{A_{0}}{2}, \quad F_{L}=\frac{1}{4}\left(2-A_{0} \mp A_{4}\right), \quad F_{R}=\frac{1}{4}\left(2-A_{0} \pm A_{4}\right),
$$

for $W^{ \pm}$, respectively.

In figures 14 and 15 we plot the longitudinal, and the difference of the left- and righthanded polarization fractions of the $W^{+}$boson, for $p_{T}^{W}>p_{T \text { cut }}^{W}$. We show these quantities for the pure SM and for the SM modified by one of the dimension-six operators in eq. (9.10). For the Wilson coefficients we use the same values as used in figure 13 such that the signal strength at $14 \mathrm{TeV}$ is modified by $40 \%$. As can be glimpsed from the figures, within the $\mathrm{SM}$ the W-boson becomes increasingly polarized in the longitudinal direction as the cut on the $W$ transverse momentum increases [191]. This behavior is not significantly affected by a right-handed current, $\xi_{u d}$, or a gauge-invariant correction to the left-handed current, $\delta V_{u d}$. The operator $c_{W W}$ would also preferentially induce a longitudinally polarized $W$ at large $p_{T \text { cut }}^{W}$, but with a smaller fraction.

On the other hand, dipole couplings of the $W$ boson to the up and down quarks would greatly reduce the longitudinal fraction at large $p_{T}$. In figure 15 we show that $\tilde{c}_{W u}$ and $\tilde{c}_{W d}$ induce, respectively, a left- and right-handed polarized $W$. Finally, a nonzero value of $\tilde{c}_{W W}$ would also reduce the longitudinal fraction and produce equal amount of left- and right-polarized $W$ bosons at large $p_{T}$. The $W$-boson helicity fractions would therefore make it possible to identify the effects of the dipole interactions, or perhaps of $\tilde{c}_{W W}$, but they would not clearly identify a right-handed current.

We now turn to the azimuthal-angle distribution which does turn out to be sensitive to RHCC. In figure 16 we show the normalized differential cross section with respect to $\phi^{*}$, with no cut on $p_{T}^{W}$, for the SM, and for the operators $\xi_{u d}, \delta V_{u d}, c_{W W}$, and $\tilde{c}_{W W}$. In the SM, the cross section is well described by the $\cos \phi^{*}$ term, with a smaller component proportional to $\cos 2 \phi^{*}$. We observe that the left-handed current, $\delta V_{u d}$, does not significantly affect the shape of the $\phi^{*}$ distribution. $c_{W W}$ induces a slightly larger $\cos 2 \phi^{*}$ component which only mildly modifies the distribution. $\xi_{u d}$ does not modify the functional form of the distribution, which is proportional to $\cos \phi^{*}$, but significantly affects the amplitude. This is captured by the coefficient $A_{3}$, which we show in the left-panel of figure 17 as a function 

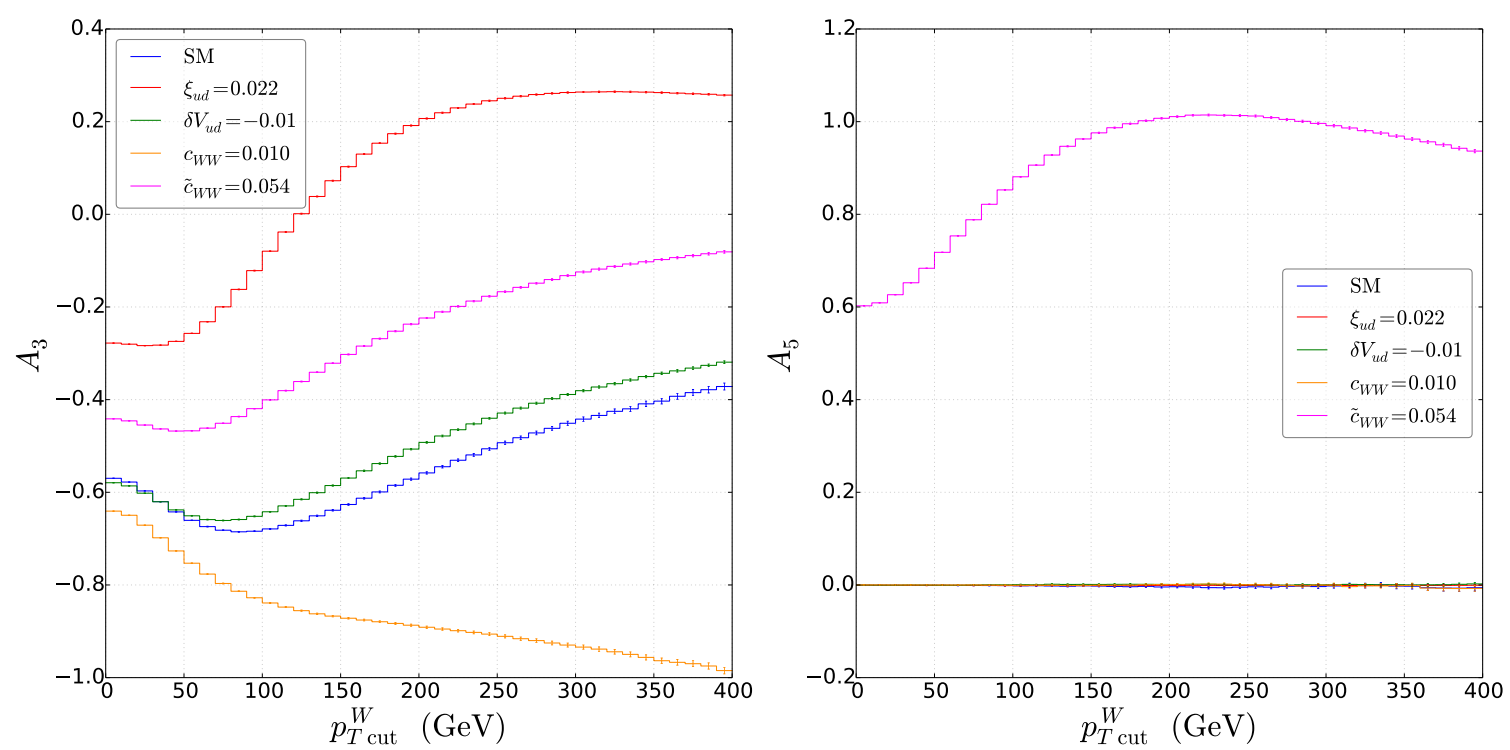

Figure 17. $A_{3}$ and $A_{5}$ coefficients in $W^{+} H$ production as a function of $p_{T \text { cut }}^{W}$, at $\sqrt{S}=14 \mathrm{TeV}$.

of $p_{T \text { cut }}^{W} \cdot A_{3}$ vanishes if parity is conserved in the production of the $W$ boson, and, for the chosen value of $\xi_{u d}$, this effectively occurs at $p_{T \text { cut }}^{W}=120 \mathrm{GeV}$. For larger cuts, $\xi_{u d}$ overtakes the SM contribution and the sign of $A_{3}$ flips.

Figure 16 shows another interesting feature, namely that $\tilde{c}_{W W}$ induces a $\sin \phi^{*}$ dependence of the cross section. Differently from all other operators in eq. (9.10), for $\tilde{c}_{W W}$ a CPV interference term with the SM survives. This term does not induce any corrections to the total cross section, but it does induce a large $A_{5}$ coefficient as shown in the right-panel of figure 17. Such a signature is a distinctive feature of a $\varphi^{\dagger} \varphi \tilde{W} W$ operator at the LHC.

This discussion shows that, in the presence of a deviation of the total $W H$ cross section from the SM prediction, a study of the $p_{T}^{W}$ spectrum and of angular distributions of the charged lepton produced by $W$ decay, would provide important information in identifying the origin of the discrepancy. We should point out that our preliminary study did not include detector effects, background estimates, nor reconstruction efficiencies and a more careful investigation is warranted. Such an investigation would be better performed within the framework of an experimental collaboration.

\subsection{Anomalous $W t b$ couplings}

Analogously to the ud-us sector it is interesting to see to what extent the constraints in the $t b$ sector are affected by turning on additional operators. To explore this, we extend our Lagrangian, involving right-handed $(t b)$ currents, with the following set of operators,

$$
\begin{aligned}
& O_{L L}=\frac{g v^{2}}{2}\left[\frac{1}{\sqrt{2}} \bar{t}_{L} \gamma^{\mu} b_{L}^{\prime} W_{\mu}^{+}+\frac{1}{\sqrt{2}} \bar{b}_{L}^{\prime} \gamma^{\mu} t_{L} W_{\mu}^{-}+\frac{1}{c_{W}} Z_{\mu} \bar{t}_{L} \gamma^{\mu} t_{L}\right]\left(1+\frac{h}{v}\right)^{2} \\
& O_{W t}=-g m_{t}\left[\frac{1}{\sqrt{2}} \bar{b}_{L}^{\prime} \sigma^{\mu \nu} t_{R} W_{\mu \nu}^{-}+\bar{t}_{L} \sigma^{\mu \nu} t_{R}\left(\frac{1}{2 c_{W}} Z_{\mu \nu}+i g W_{\mu}^{-} W_{\nu}^{+}\right)\right]\left(1+\frac{h}{v}\right),
\end{aligned}
$$




\begin{tabular}{|c|c|c|c|c|c|}
\hline Real & Individual & Marginalized & Imaginary & Individual & Marginalized \\
\hline$\xi_{t b}$ & {$[-1.4,1.5] \cdot 10^{-3}$} & {$[-0.01,0.12]$} & $\xi_{t b}$ & {$[-2.4,2.4] \cdot 10^{-3}$} & {$[-0.16,0.13]$} \\
$\delta_{L L}$ & {$[-0.03,0.04]$} & {$[-0.03,0.04]$} & - & & \\
$v^{2} C_{W t}$ & {$[-0.09,0.05]$} & {$[-0.10,0.04]$} & $v^{2} C_{W t}$ & {$[-6.0,6.0] \cdot 10^{-4}$} & {$[-1.0,0.9] \cdot 10^{-3}$} \\
$v^{2} C_{W b}$ & {$[-0.04,0.05]$} & {$[-3.5,0.4]$} & $v^{2} C_{W b}$ & {$[-3.5,3.5] \cdot 10^{-2}$} & {$[-1.9,2.2]$} \\
\hline
\end{tabular}

Table 12. Allowed regions (90\% C.L.) for the $W t b$ couplings at the scale $\Lambda=1 \mathrm{TeV}$. The second and fifth columns show the constraints under the assumption that only a single coupling is generated at the high scale. In the third and sixth columns we assume that all Wtb couplings in eq. (9.17) are present at the scale of new physics and we marginalize over all couplings.

$$
O_{W b}=-g m_{b}\left[\frac{1}{\sqrt{2}} \bar{t}_{L}^{\prime} \sigma^{\mu \nu} b_{R} W_{\mu \nu}^{+}-\bar{b}_{L} \sigma^{\mu \nu} b_{R}\left(\frac{1}{2 c_{W}} Z_{\mu \nu}+i g W_{\mu}^{-} W_{\nu}^{+}\right)\right]\left(1+\frac{h}{v}\right),
$$

which appear in the Lagrangian with couplings $C_{L L, W t, W b}$, respectively. Here, $b^{\prime}=V_{t b} b+$ $V_{t s} s+V_{t d} d, t^{\prime}=V_{t b}^{*} t+V_{c b}^{*} c+V_{u b}^{*} u$, and $c_{W}=\cos \theta_{W}$, with $\theta_{W}$ the Weinberg angle. In total, the effective $W t b$ vertex can then be written as

$$
\mathcal{L}_{t b}=\frac{g}{\sqrt{2}} \bar{t}\left[\gamma^{\mu}\left(V_{t b}\left(1+\delta_{L L}\right) P_{L}+\xi_{t b} P_{R}\right) W_{\mu}^{+}-\sigma^{\mu \nu} W_{\mu \nu}^{+}\left(m_{t} C_{W t}^{*} P_{L}+m_{b} C_{W b} P_{R}\right)\right] b+\text { h.c. },
$$

where $^{6} \delta_{L L}=\frac{v^{2}}{2} C_{L L}$. Eq. (9.18) provides a general parametrization of the $W t b$ vertex [193] and these couplings have been studied in many previous works [79, 192-205]. Most of these studies constrain the $W t b$ vertex by looking at collider processes or flavor constraints (in particular $\Delta B=1$ processes). Here we study the effect of turning on $\xi_{t b}$ and $C_{L L, W t, W b}$ simultaneously, while taking into account both collider and low-energy constraints including those from EDM experiments which are usually not considered.

To derive the resulting constraints we require several additions to the expressions discussed in previous sections. In particular, for the $C_{L L, W b, W t}$ contributions to the helicity fractions discussed in section 2.4, we employ the expressions given in ref. [79]. We take into account $\delta_{L L}$ contributions to single-top production by replacing $V_{t q} \rightarrow V_{t q}\left(1+\delta_{L L}\right)$, where $q \in(d, s, b)$. For the running and matching of $C_{L L, W t, W b}$ onto the $C_{7,8}$ operators relevant for $b \rightarrow q \gamma$, we use the expressions in refs. [192, 206]. Finally, for the contributions of $C_{W t}$ and $C_{W b}$ to EDMs we follow the analysis of refs. [207, 208]. With this combined input we turn on $\delta_{L L}, \xi_{t b}$, and $C_{W t, W b}$ simultaneously, while setting $V_{t b}=1$. The resulting constraints are shown in table 12, together with the bounds that result from a single-coupling analysis.

The constraints on $\delta_{L L}$ and $C_{W t}$ are the least affected by the presence of the other operators, and the marginalized bounds are fairly close to the single-coupling analysis. This can be understood by noticing that $\delta_{L L}$ is stringently constrained by single-top production, which does not allow for cancellations against the other couplings. Similarly, $\operatorname{Im} C_{W t}$

\footnotetext{
${ }^{6}$ Within the framework of the SMEFT, $O_{L L}$ arises from the operators $Q_{H q}^{(1)}$ and $Q_{H q}^{(3)}$ in the notation of [42, 43, 80]. We follow ref. [192] and assume no flavor-changing neutral currents at tree level, the $W t b$ coupling is then hermitian and $C_{L L}$ is forced to be real.
} 

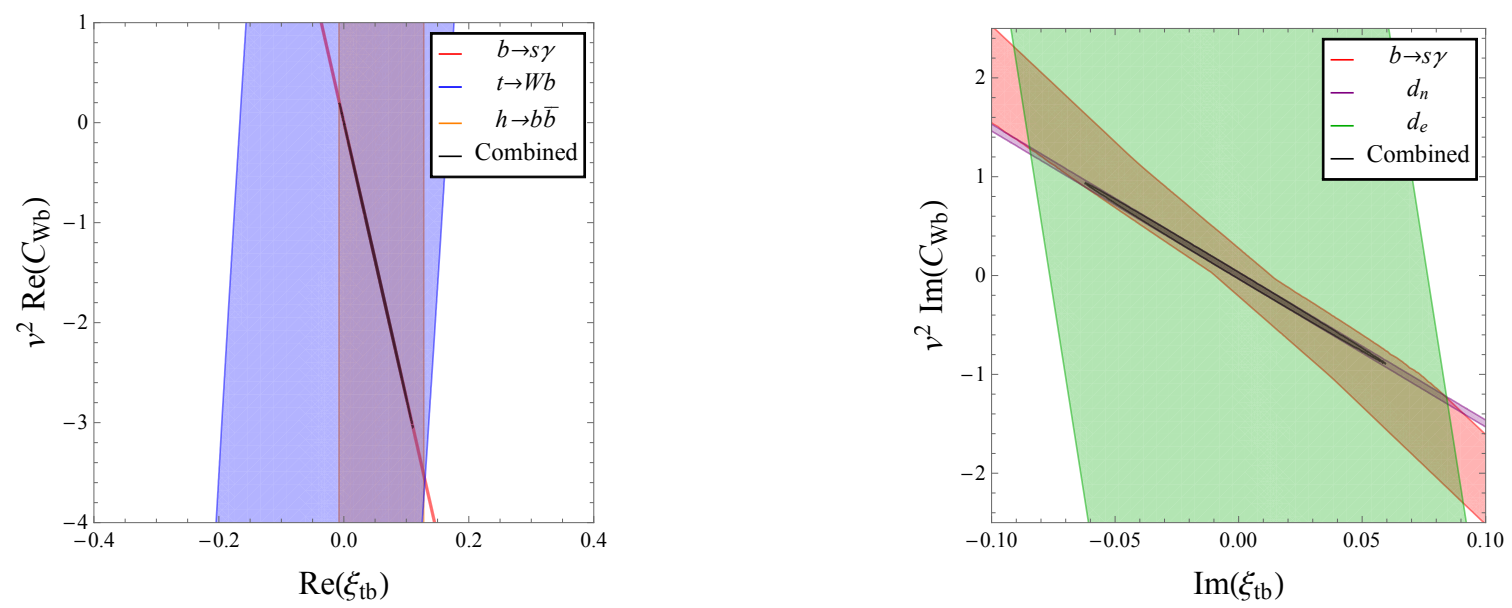

Figure 18. The figure shows the constraints in the $\xi_{t b}-v^{2} C_{W b}$ plane, illustrating the significant cancellations that can occur between the two couplings. The real (imaginary) couplings are shown in the left (right) panel. The exclusion bands (at 90\% C.L.) assume that only $C_{W b}$ and $\xi_{t b}$ are generated at the scale $\Lambda$.

provides the dominant contribution to the electron EDM such that its constraint survives to large extent the global analysis as well. $\operatorname{Re} C_{W t}$ is constrained by several observables with similar strength (electroweak precision tests, W helicity fractions, and $b \rightarrow s \gamma$, see ref. [208]) such that the marginalized constraint is not too different from the individual one.

The situation is significantly different for $C_{W b}$ and $\xi_{t b}$. In the single-coupling analysis the real parts of these couplings are mainly constrained by $b \rightarrow s \gamma$, while their imaginary parts are constrained by the neutron EDM. When both operators are present the constraints on the real and imaginary parts can be weakened significantly by mutual cancellations in $\mathrm{BR}(b \rightarrow s \gamma)$ and $d_{n}$, respectively. In fact, comparing the second (fifth) and third (sixth) columns of table 12 we see that the limits on the real (imaginary) part of $\xi_{t b}$ deteriorate by roughly two orders of magnitude. The bounds are similarly weakened for $C_{W b}$.

This effect is illustrated in figure 18, where we show the constraints in the $\xi_{t b}-C_{W b}$ plane for the real and imaginary parts. The results in this figure assumes only $\xi_{t b}$ and $C_{W b}$ to be present at the scale $\Lambda$, but this is sufficient to see that significant cancellations can occur between these two couplings. The left-panel shows that the CP-even $b \rightarrow s \gamma$ observables allow for a free direction, and the much weaker limits from the helicity fractions and $h \rightarrow b \bar{b}$ are needed to obtain a constraint. In the case of the imaginary parts, shown in the right panel, the neutron EDM allows for a free direction and the electron EDM is needed to obtain a bound. As can be seen from table 12, including $C_{W t}$ and $\delta_{L L}$ hardly affects the bounds on the real parts of $\xi_{t b}$ and $C_{W b}$ compared to figure 18. The limits on the imaginary parts are weakened by a minor factor compared to figure 18, confirming that the major deterioration between the single-coupling and global constraints are indeed due to cancellations between $\xi_{t b}$ and $C_{W b}$. A comparison of the single-coupling and global constraints for the $\operatorname{Re} \xi_{t b}-\operatorname{Im} \xi_{t b}$ plane is shown in figure 19. Again, it is clear that turning on several couplings can severely weaken the various constraints. 


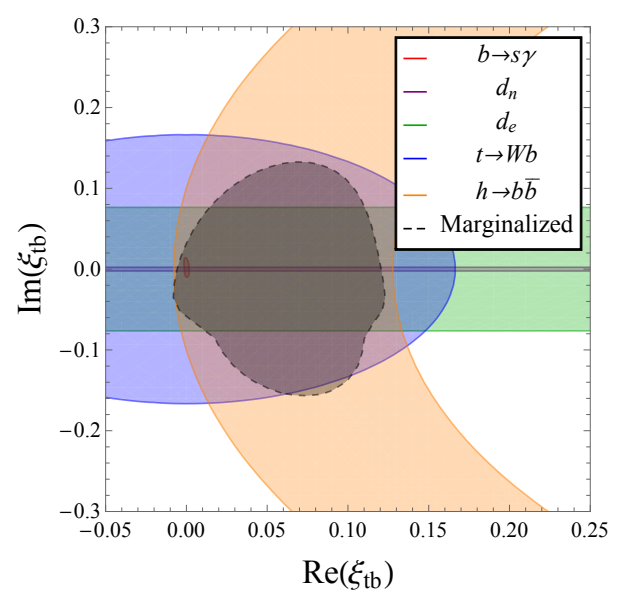

Figure 19. The figure shows the constraints in the $\operatorname{Re} \xi_{t b}-\operatorname{Im} \xi_{t b}$ plane. The constraints from $b \rightarrow s \gamma, d_{n}, d_{e}$, the helicity fractions, and $h \rightarrow b \bar{b}$ are shown in red, purple, green, blue, and orange, respectively, and assume that only $\xi_{t b}$ is generated at the scale $\Lambda$. The dashed black line is the resulting constraint when marginalizing over the other $t b$ couplings.

In summary, in the $t b$ sector isolating the right-handed current is complicated by the degeneracy with the $C_{W b}$ dipole operator. Nevertheless, as shown in table 9.3, the marginalized constraints on most of the anomalous $W t b$ operators are very stringent.

\section{Conclusion}

Motivated by the attractive possibility of parity restoration at high energies, we investigated possible footprints left behind by a right-handed extension of the SM. We studied in detail the right-handed charged-current couplings $\xi_{i j}$ defined in eq. (1.1), looking at their manifestations in collider experiments, flavor physics, and low-energy precision tests.

Our work provides a case study of the complementarity of low-energy and collider experiments in probing heavy new physics which is not within direct reach of the LHC, and therefore can be analyzed in the framework of the SMEFT.

In a first major thrust of our work, assuming that at the scale $\Lambda$ the SM is modified dominantly by the RHCC operator, we have worked out the bounds on the $\xi_{i j}$ from a broad range of probes. The resulting $90 \%$ C.L. limits are summarized in eqs. (2.27) (collider) and (8.1), (8.2) (global fit). A graphical summary is presented in figure 10. Note that in this setup one can straightforwardly compare the sensitivity of various direct and indirect probes both at high and low energy. Such a comparison reveals that low-energy probes provide the strongest constraints, putting most of the $\xi_{i j}$ out of LHC sensitivity reach.

The above results, however, should be put in a broader context. Since most explicit models of new physics generate more than one class of operators at the UV-matching scale $\Lambda$, many of the observables used in our analysis would receive contributions form several other dimension-six operators in the SMEFT. Therefore, in a more general setting, the low-energy constraints require that certain linear combinations of dimension-six Wilson coefficients (including the $\xi_{i j}$ ) be highly constrained, which in turn imposes non-trivial 
constraints on new physics scenarios. Realizing this, in a second thrust of our work we have explored: (i) the impact of degeneracies on the $\xi_{i j}$, finding that the low-energy bounds can be weakened to a level comparable to the collider sensitivity by turning on additional operators; (ii) ways to remove this degeneracy, identifying observables that would uniquely point to RHCC, both at collider and low-energy. Details can be found in section 9, with focus on the couplings $\xi_{u d}, \xi_{u s}$, and $\xi_{t b}$. Our analysis shows the importance of pursuing improved searches of $\xi_{i j}$ manifestations at both the energy and precision frontiers, and suggests new handles on RHCC at colliders.

We conclude by listing the main highlights of our analysis:

- Keeping in mind the significant theoretical uncertainties, we note that the introduction of appropriate $\xi_{i j}$ elements can help resolve some tensions between data and SM predictions in flavor physics, such as $\epsilon^{\prime} / \epsilon$ [11] and the inclusive-exclusive discrepancy in $V_{u b}$ and $V_{c b}[209,210]$.

- In the framework of the linearly realized SMEFT, the most stringent collider constraint on the light elements $\xi_{i j}$, with $i \in\{u, c\}$ and $j \in\{d, s, b\}$, come from the associated production of a Higgs and a $W$ boson, followed by $W$ production and Higgs production via vector boson fusion. The right-handed charged-current operator $\xi$ also affects $W Z$ production, which, at the moment, provides somewhat weaker bounds.

- Nucleon beta decay, and leptonic and semileptonic decays of pion, kaons, and $D$ mesons allow one to obtain strong bounds on the elements $\xi_{u d}, \xi_{u s}, \xi_{c d}$, and $\xi_{c s}$. Under the assumption that the SM is modified solely by the RHCC operator $\xi$, the low-energy bounds put on $\xi_{u d}$ and $\xi_{u s}$ are out of the collider reach, while in the case of $\xi_{c d}$ and $\xi_{c s}$ improved constraints from the LHC Run II can compete with low-energy bounds. If we allow for modifications to the couplings of left-handed quarks to the $W$ boson, or for additional semileptonic operators, the single coupling bounds on $\xi_{u d}$ can be weakened to the percent level, see figure 12, making it important to look for collider constraints on this coupling.

- To this end, we identified differential distributions in $W H$ production which are very sensitive to the Lorentz structure of the coupling of the light quarks to the $W$ boson. In the presence of a deviation from the SM expectations, these distributions could help identify the possible origin of the correction, disentangling RHCC interactions from other possible modifications of the $W H$ process (see for example figure 16).

- At colliders, it is hard to probe $\xi_{u b}$ and $\xi_{c b}$ at a level comparable to the one achievable in exclusive and inclusive $B$ decays. Possible strategies might involve tagging $b$ jets in $W H$ and $V B F$, but in both cases it would remain hard to access values of $\xi_{u b}$ and $\xi_{c b}$ smaller than the corresponding CKM elements. We observe that, even including flavor observables, the bounds on $\xi_{u b}$ and $\xi_{c b}$ are not extremely strong. In light of this, a more detailed study of RH contributions to inclusive $B$-meson decays might be appropriate. Such a study might also resolve whether RHCCs can explain the current tension between the determinations from exclusive and inclusive $B$ decays. 
- The collider observables that are needed to constrain the third row of the $\xi$ matrix are single-top production, top decays, with particular attention to the $W$ polarization in the decay, and $h \rightarrow b \bar{b}$. It is quite interesting that the loop process $h \rightarrow b \bar{b}$ already probes $\xi_{t b}$ at a level comparable to top decays.

- Right-handed currents in the top sector are, however, severely constrained by $B \rightarrow X_{s, d} \gamma$ and EDMs. In a single-coupling analysis, the limits are two to three orders of magnitude stronger than collider limits, and are not severely weakened by turning on $\xi_{t d}, \xi_{t s}$, and $\xi_{t b}$ at the same time. The collider limits become relevant only if we allow for more general modifications of the $W t b$ vertex. In section 9.3 we therefore performed a global analysis, including all relevant low- and high-energy experiments, of the most general modification of the $W t b$ interactions. In such a scenario, $\xi_{t b}$ and $C_{W b}$ are strongly correlated (see figure 18) and the resulting limits are significantly softened. Our analysis extends those based on subsets of the available data, see e.g. refs. [196, 197, 205], and reflects the relevance of low-energy precision experiments.

- Despite the large theoretical uncertainties, limits on EDMs provide strong constraints on the imaginary parts of many $\xi$ elements and even on some of the real parts because of the interplay with imaginary parts of certain CKM elements. Improvements of hadronic and nuclear theory could further strengthen the constraining power of EDM experiments as can be seen from figure 10. In the ideal case of negligible theoretical uncertainties, EDM experiments would set the strongest constraints on all imaginary parts (except for $\left.\operatorname{Im} \xi_{b c}\right)$, reaching the $\mathcal{O}\left(10^{3} \mathrm{TeV}\right)$ scale for $\operatorname{Im} \xi_{u d}$, and the real parts of $\xi_{u b}, \xi_{c d}$, and $\xi_{t d}$.

\section{Acknowledgments}

SA acknowledges support by the COFUND Fellowship under grant agreement PCOFUNDGA-2012-600377. VC and EM acknowledge support by the US DOE Office of Nuclear Physics and by the LDRD program at Los Alamos National Laboratory. WD and JdV acknowledge support by the Dutch Organization for Scientific Research (NWO) through a RUBICON and VENI grant, respectively. We thank Paolo Gambino for discussion on the inclusive decays $B \rightarrow X_{c} l \nu$ and for providing us his code for the evaluation of the leptonic moments of the inclusive decay distribution. We thank Mikolaj Misiak for providing us the generalization of the anomalous dimension (3.10) to generic values of the charges $Q_{u}$ and $Q_{d}$. We acknowledge several useful discussions with Zoltan Ligeti, Giuseppe Cerati, Andreas Crivellin, Ivo van Vulpen, and Marc de Beurs. We thank U. Egede, M. GonzálezAlonso, and N. Yamanaka for comments on the manuscript.

\section{A Additional input for CKM fits}

In this appendix we discuss several processes that get small contributions from RHCCs, but that were used in the fits discussed in section 8 to constrain the SM CKM elements. In appendix A.1 we discuss $B \rightarrow J / \psi K$, which determines the CKM angle $\beta$. RHCC in the 
$W b c$ vertex contribute to this observable at tree level, but, as we will argue, the contribution is suppressed with respect to the SM. The remaining elements of the $\xi_{i j}$ matrix generate no or small contributions, after taking into account the limits from other observables.

In appendix A.2 we discuss the FCNC decays $B_{q} \rightarrow \mu^{+} \mu^{-}, K_{L} \rightarrow \pi^{0} \nu \nu$, and the penguin contributions to $K_{L} \rightarrow \pi^{0} e^{+} e^{-}$. These contributions are quadratic in $\xi$ and necessarily involve two different $\xi$ elements. Thus, they do not play any role in a single coupling analysis. Finally, in appendix A.3 we discuss $\Delta F=2$ processes. Also in this case, contributions are quadratic in $\xi$. Both types of processes might play a more important role in a global analysis involving all $\xi_{i j}$ couplings, as possible cancellations may allow for larger values of the $\xi_{i j}$ couplings. However, this is beyond the scope of the current work, and we neglect all dimension-eight effects discussed in these appendices.

\section{A.1 $\quad B \rightarrow J / \psi K$}

The CKM angle $\beta=\arg \left(-\frac{V_{c d} V_{c b}^{*}}{V_{t d} V_{t b}^{*}}\right)$ is determined from $S_{J / \psi K}$ which appears in the CP asymmetry,

$$
\frac{\Gamma(\bar{B} \rightarrow J / \psi K)-\Gamma(B \rightarrow J / \psi K)}{\Gamma(\bar{B} \rightarrow J / \psi K)+\Gamma(B \rightarrow J / \psi K)}=S_{J / \psi K} \sin \left(\Delta m_{d} t\right)+C_{J / \psi K} \cos \left(\Delta m_{d} t\right)
$$

Here

$$
S_{J / \psi K}=\frac{2 \operatorname{Im} \lambda_{J / \psi K}}{1+\left|\lambda_{J / \psi K}\right|^{2}}, \quad \lambda_{J / \psi K}=\frac{q}{p} \frac{\bar{A}_{J / \psi K}}{A_{J / \psi K}}=\frac{V_{t b}^{*} V_{t d}}{V_{t b} V_{t d}^{*}} \frac{\bar{A}_{J / \psi K}}{A_{J / \psi K}},
$$

where the ratio $\frac{q}{p}=\frac{V_{t b}^{*} V_{t d}}{V_{t b} V_{t d}^{*}}$ is due to the $\bar{B}-B$ mixing and $A_{J / \psi K}\left(\bar{A}_{J / \psi K}\right)$ is the amplitude for the decay $B \rightarrow J / \psi K(\bar{B} \rightarrow J / \psi K)$. In the $\mathrm{SM}$, this amplitude is due to a treelevel decay (proportional to $V_{c b} V_{c s}^{*}$ ) followed by $\bar{K}-K$ mixing (the real part of which is dominated by a term proportional to $V_{c s} V_{c d}^{*}$ ), such that to good approximation we have,

$$
\frac{\bar{A}_{J / \psi K}}{A_{J / \psi K}}=\frac{V_{c b} V_{c d}^{*}}{V_{c b}^{*} V_{c d}}, \quad S_{J / \psi K}=\sin 2 \beta .
$$

The experimental value is given by [71],

$$
S_{J / \psi K}=0.682 \pm 0.019 .
$$

RHCCs can contribute to this observable through the amplitude, $A_{J / \psi K}$, or through $B$ or $K$ mixing. As discussed in appendix A.3, the RHCC contributions to meson mixing are quadratic in $\xi$, and we neglect them here. The RHCC contributions to the amplitude can arise from $\xi_{c s}$ and $\xi_{c b}$. The constraints on $\xi_{c s}$ from semileptonic $D$ and $D_{s}$ decays imply that the contribution to $S_{J / \psi K}$ is at the percent level and therefore negligible. As for $\xi_{c b}$, the corrections to $S_{J / \psi K}$ are proportional to $\xi_{c b} / V_{c b}$ and to ratios of the matrix elements of the left-right and SM operators, $\mathcal{O}_{1,2 L R}^{c b c s}$ and $\mathcal{O}_{1,2 L L}^{c b c s}$. In this case, inclusive and exclusive $B$ decays into charmed final states and $B_{q}-\bar{B}_{q}$ oscillations allow for relatively large values of $\operatorname{Im}\left(\xi_{c b}\right) / V_{c b}, \operatorname{Im}\left(\xi_{c b}\right) / V_{c b} \sim 0.3$. For exclusive $B$ decays into $s$-wave charmonia, it was shown that the matrix elements factorize [211-213]. In particular, in the case of the left-right operators, one has to estimate the matrix element $\left\langle J / \psi\left|\bar{c}_{L} c_{R}\right| 0\right\rangle$. While a precise evaluation 
is difficult, we notice that both in the nonrelativistic and in the $m_{c} \rightarrow 0$ limit, the matrix element vanishes at leading order. We therefore expect the corrections to $A_{J / \psi K}$ to be relatively small. Since the suppression factors in the two limits, the relative velocity of the charm quarks in the $J / \psi$ or the ratio $m_{J / \psi} / m_{B}$, are not extremely small, it might nonetheless be worthwhile to more rigorously investigate RHCC contributions to $B \rightarrow J / \psi K$.

\section{A.2 $\Delta F=1$ neutral current decays}

The effective Hamiltonian for $B_{s, d}^{0} \rightarrow \mu^{+} \mu^{-}, K_{L} \rightarrow \mu^{+} \mu^{-}, K_{L} \rightarrow \pi^{0} e^{+} e^{-}$and $K_{L} \rightarrow \pi^{0} \nu \nu$ contains the semileptonic operators

$$
\begin{aligned}
\mathcal{H}= & -\frac{G_{F}^{2} m_{W}^{2}}{16 \pi^{2}}\left\{C_{L V}^{i j} \bar{d}^{j} \gamma^{\mu} P_{L} d^{i} \bar{l} \gamma_{\mu} l+C_{R V}^{i j} \bar{d}^{j} \gamma^{\mu} P_{R} d^{i} \bar{l} \gamma_{\mu} l+C_{L l l}^{i j} \bar{d}^{j} \gamma^{\mu} P_{L} d^{i} \bar{l} \gamma_{\mu} P_{L} l\right. \\
& \left.+C_{R l l}^{i j} \bar{d}^{j} \gamma^{\mu} P_{R} d^{i} \bar{l} \gamma_{\mu} P_{L} l+C_{L \nu \nu}^{i j} \bar{d}^{j} \gamma^{\mu} P_{L} d^{i} \bar{\nu} \gamma_{\mu} P_{L} \nu+C_{R \nu \nu}^{i j} \bar{d}^{j} \gamma^{\mu} P_{R} d^{i} \bar{\nu} \gamma_{\mu} P_{L} \nu\right\}
\end{aligned}
$$

The matching coefficients at the scale $\mu=m_{W}$ are obtained by computing penguin and box diagrams, and, for $i \neq j$, we find in the $\overline{\mathrm{MS}}$ scheme

$$
\begin{aligned}
& C_{L V}^{i j}=V_{t j}^{*} V_{t i} s_{w}^{2}\left\{\frac{8\left(8-50 x_{t}+63 x_{t}^{2}+6 x_{t}^{3}-24 x_{t}^{4}\right)}{9\left(x_{t}-1\right)^{4}} \log x_{t}\right. \\
&\left.+\frac{4 x_{t}\left(108-259 x_{t}+163 x_{t}^{2}-18 x_{t}^{3}\right)}{9\left(x_{t}-1\right)^{3}}\right\}, \\
& C_{R V}^{i j}= \xi_{t j}^{*} \xi_{t i} s_{w}^{2}\left\{\frac{32}{3}\left(2-3 x_{t}\right) \log \frac{\mu^{2}}{m_{W}^{2}}+\frac{8\left(8-14 x_{t}-81 x_{t}^{2}+222 x_{t}^{3}-168 x_{t}^{4}+36 x_{t}^{5}\right)}{9\left(x_{t}-1\right)^{4}} \log x_{t}\right. \\
&\left.-\frac{4\left(-320+528 x_{t}+141 x_{t}^{2}-493 x_{t}^{3}+162 x_{t}^{4}\right)}{27\left(x_{t}-1\right)^{3}}\right\} \\
&-\left(\frac{1472}{27}-\frac{256}{9} \log \frac{\mu^{2}}{m_{W}^{2}}\right) s_{w}^{2}\left(\xi_{c j}^{*} \xi_{c i}+\xi_{u j}^{*} \xi_{u i}\right), \\
& C_{L l l}^{i j}= V_{t j}^{*} V_{t i}\left\{\frac{12 x_{t}^{2}}{\left(x_{t}-1\right)^{2}} \log x_{t}+\frac{4 x_{t}\left(x_{t}-4\right)}{x_{t}-1}\right\}, \\
& C_{R l l}^{i j}= \xi_{t j}^{*} \xi_{t i}\left\{4\left(-3+4 x_{t}\right) \log \frac{\mu^{2}}{m_{W}^{2}}-\frac{4 x_{t}\left(10-11 x_{t}+4 x_{t}^{2}\right)}{\left(x_{t}-1\right)^{2}} \log x_{t}+4 \frac{4-4 x_{t}+3 x_{t}^{2}}{x_{t}-1}\right\} \\
&-\left(16+12 \log \frac{\mu^{2}}{m_{W}^{2}}\right)\left(\xi_{c j}^{*} \xi_{c i}+\xi_{u j}^{*} \xi_{u i}\right), \\
& C_{L \nu \nu}^{i j}= V_{t j}^{*} V_{t i}\left\{-12 \frac{x_{t}\left(x_{t}-2\right)}{\left(1-x_{t}\right)^{2}} \log x_{t}-4 x_{t} \frac{2+x_{t}}{x_{t}-1}\right\}, \\
& C_{R \nu \nu}^{i j}= \xi_{t j}^{*} \xi_{t i}\left\{4\left(3-4 x_{t}\right) \log \frac{\mu^{2}}{m_{W}^{2}}+4 x_{t} \frac{4-11 x_{t}+4 x_{t}^{2}}{\left(1-x_{t}\right)^{2}} \log x_{t}+4 \frac{2+4 x_{t}-3 x_{t}^{2}}{x_{t}-1}\right\} \\
&-\left(8-12 \log \frac{\mu^{2}}{m_{W}^{2}}\right)\left(\xi_{c j}^{*} \xi_{c i}+\xi_{u j}^{*} \xi_{u i}\right),
\end{aligned}
$$

where we neglected powers of $x_{c}$ and $x_{u}$ and we used unitarity for the SM contributions.

Of the above operators, $C_{L l l}^{b q}$ and $C_{R l l}^{b q}$ contribute to $B_{s, d}^{0} \rightarrow l^{+} l^{-}$and $K_{L} \rightarrow \mu^{+} \mu^{-}$. The photon penguins $C_{L V}^{i j}$ and $C_{R V}^{i j}$ do not contribute due to vector current conservation. 
The decay rate is

$$
\Gamma\left(B_{q}^{0} \rightarrow l^{+} l^{-}\right)=\frac{1}{32 \pi} \sqrt{1-\frac{4 m_{l}^{2}}{m_{B_{q}}^{2}}} m_{B_{q}} f_{B_{q}}^{2} m_{l}^{2}\left(\frac{G_{F}^{2} m_{W}^{2}}{16 \pi^{2}}\right)^{2}\left|C_{L l l}^{b q}-C_{R l l}^{b q}\right|^{2},
$$

where the minus sign between the Wilson coefficients is due to the fact that only the axial part of the quark current contributes. The observed branching ratios for $B_{d, s} \rightarrow \mu^{+} \mu^{-}$ are $[101]$

$$
\mathrm{BR}\left(B_{d}^{0} \rightarrow \mu^{+} \mu^{-}\right)=\left(3.9_{-1.4}^{+1.6}\right) \cdot 10^{-10}, \quad \mathrm{BR}\left(B_{s} \rightarrow \mu^{+} \mu^{-}\right)=\left(2.8_{-0.6}^{+0.7}\right) \cdot 10^{-9} .
$$

From eq. (A.6) one sees that RHCC contributions to these processes are relevant if $\xi_{t b}^{*} \xi_{t q} \sim$ $V_{t b}^{*} V_{t q}$. As discussed in section 8, this possibility is ruled out by $B \rightarrow X_{s, d} \gamma$, even when all $\xi_{t j}$ are turned on at the same time. Similarly, the RHCC contributions to $K_{L} \rightarrow \mu^{+} \mu^{-}$are small after taking into account limits from semileptonic decays and $B \rightarrow X_{q} \gamma$. In our analysis we therefore only use $\operatorname{BR}\left(B_{s, d}^{0} \rightarrow \mu^{+} \mu^{-}\right)$to constrain the CKM elements $V_{t s}$ and $V_{t d}$.

$C_{L \nu \nu}^{s d}$ and $C_{R \nu \nu}^{s d}$ contribute to the decay $K_{L} \rightarrow \pi^{0} \nu \bar{\nu}$. The contributions of the SM and RHCC are of similar size if $\operatorname{Im}\left(\xi_{t s}^{*} \xi_{t d}\right) \sim \operatorname{Im}\left(V_{t s}^{*} V_{t d}\right) \sim 10^{-4}$, which is ruled out by EDMs, and by the branching ratio and $\mathrm{CP}$ asymmetry in $B \rightarrow X_{s, d} \gamma$. Therefore, this channel might become interesting only in scenarios in which multiple operators are turned on at the same time.

The operators $C_{L V}^{s d}$ and $C_{L \mu \mu}^{s d}$ give the leading SM contribution to $K_{L} \rightarrow \pi^{0} e^{+} e^{-}$. They are related to the operators $C_{7 V}$ and $C_{7 A}$ defined in ref. [85] by

$$
C_{7 V}=\frac{\alpha_{\mathrm{em}}}{32 \pi s_{w}^{2}}\left(C_{L V}^{d s}+\frac{C_{L \mu \mu}^{d s}}{2}\right), \quad C_{7 A}=-\frac{\alpha_{\mathrm{em}}}{64 \pi s_{w}^{2}} C_{L \mu \mu}^{d s}
$$

$C_{7 A}$ does not run, while $C_{7 V}$ mixes with tree-level charged currents. Factoring out a factor of $\alpha_{\mathrm{em}} / 2 \pi$, the authors of ref. [85] define the couplings

$$
\tilde{y}_{7 V}(\mu)=P_{0}(\mu)-4\left(C_{0}\left(x_{t}\right)+\frac{1}{4} D_{0}\left(x_{t}\right)\right)+\frac{Y_{0}\left(x_{t}\right)}{s_{w}^{2}}, \quad \tilde{y}_{7 A}=-\frac{Y_{0}\left(x_{t}\right)}{s_{w}^{2}},
$$

with

$$
\begin{aligned}
Y_{0}\left(x_{t}\right) & =\frac{x_{t}}{8}\left(\frac{4-x_{t}}{1-x_{t}}+\frac{3 x_{t}}{\left(1-x_{t}\right)^{2}} \log x_{t}\right), \\
C_{0}\left(x_{t}\right) & =\frac{x_{t}}{8}\left(\frac{x_{t}-6}{x_{t}-1}+\frac{3 x_{t}+2}{\left(1-x_{t}\right)^{2}} \log x_{t}\right), \\
D_{0}\left(x_{t}\right) & =-\frac{4}{9} \log x_{t}+\frac{-19 x_{t}^{3}+25 x_{t}^{2}}{36\left(x_{t}-1\right)^{3}}+\frac{x_{t}^{2}\left(5 x_{t}^{2}-2 x_{t}-6\right)}{18\left(1-x_{t}\right)^{4}} \log x_{t},
\end{aligned}
$$

where $x_{t}=m_{t}^{2} / m_{W}^{2}$. Without resummation, $P_{0}=-4 / 9 \log x_{c}$. The value of $P_{0}(\mu)$ at different scales is given in ref. [85]. 


\section{A.3 $\Delta F=2$ processes}

The effective Hamiltonian for $\Delta S=2$ processes in the presence of a RHCC is given by [214]

$$
\mathcal{H}^{\mathrm{eff}}=\frac{G_{F}^{2} m_{W}^{2}}{16 \pi^{2}} \sum C_{i}(\mu) \mathcal{O}_{i}(\mu)
$$

with

$$
\begin{aligned}
\mathcal{O}_{1}^{\mathrm{VLL}} & =\left(\bar{s} \gamma_{\mu} P_{L} d\right)\left(\bar{s} \gamma^{\mu} P_{L} d\right), & \mathcal{O}_{1}^{\mathrm{VRR}} & =\left(\bar{s} \gamma_{\mu} P_{R} d\right)\left(\bar{s} \gamma^{\mu} P_{R} d\right), \\
\mathcal{O}_{1}^{\mathrm{LR}} & =\left(\bar{s} \gamma_{\mu} P_{L} d\right)\left(\bar{s} \gamma^{\mu} P_{R} d\right), & \mathcal{O}_{2}^{\mathrm{LR}} & =\left(\bar{s} P_{L} d\right)\left(\bar{s} P_{R} d\right), \\
\mathcal{O}_{1}^{\mathrm{SLL}} & =\left(\bar{s} P_{L} d\right)\left(\bar{s} P_{L} d\right), & \mathcal{O}_{1}^{\mathrm{SRR}} & =\left(\bar{s} P_{R} d\right)\left(\bar{s} P_{R} d\right), \\
\mathcal{O}_{2}^{\mathrm{SLL}} & =\left(\bar{s} \sigma^{\mu \nu} P_{L} d\right)\left(\bar{s} \sigma_{\mu \nu} P_{L} d\right) & \mathcal{O}_{2}^{\mathrm{SRR}} & =\left(\bar{s} \sigma^{\mu \nu} P_{R} d\right)\left(\bar{s} \sigma_{\mu \nu} P_{R} d\right) .
\end{aligned}
$$

An analogous Hamiltonian can be written for $\Delta B=2$ and $\Delta C=2$ processes.

The coefficients $C_{i}$ are obtained by computing the box diagrams with two $W$ exchanges, for which we find in the $\overline{\mathrm{MS}}$ scheme

$$
\begin{aligned}
C_{1}^{\mathrm{VLL}} & =V_{i s}^{*} V_{i d} V_{j s}^{*} V_{j d}\left(\left(6-x_{i}-x_{j}\right) \log \frac{\mu^{2}}{m_{W}^{2}}+f_{1}\left(x_{i}, x_{j}\right)\right) \\
C_{1}^{\mathrm{LR}} & =2 V_{i s}^{*} V_{i d} \xi_{j s}^{*} \xi_{j d}\left(\left(6-x_{i}-x_{j}\right) \log \frac{\mu^{2}}{m_{W}^{2}}+f_{2}\left(x_{i}, x_{j}\right)\right) \\
C_{2}^{\mathrm{LR}} & =2 \frac{m_{i} m_{j}}{m_{W}^{2}} \xi_{i s}^{*} V_{i d} V_{j s}^{*} \xi_{j d}\left(-4 \log \frac{\mu^{2}}{m_{W}^{2}}+f_{3}\left(x_{i}, x_{j}\right)-4 \delta_{u, c}^{i} \delta_{u, c}^{j} g\left(x_{i}, x_{j}\right)\right) \\
C_{1}^{\mathrm{SLL}} & =\frac{m_{i} m_{j}}{m_{W}^{2}} \xi_{i s}^{*} V_{i d} \xi_{j s}^{*} V_{j d}\left(-4 \log \frac{\mu^{2}}{m_{W}^{2}}+f_{3}\left(x_{i}, x_{j}\right)-4 \delta_{u, c}^{i} \delta_{u, c}^{j} g\left(x_{i}, x_{j}\right)\right) \\
C_{1}^{\mathrm{SRR}} & =\frac{m_{i} m_{j}}{m_{W}^{2}} V_{i s}^{*} \xi_{i d} V_{j s}^{*} \xi_{j d}\left(-4 \log \frac{\mu^{2}}{m_{W}^{2}}+f_{3}\left(x_{i}, x_{j}\right)-4 \delta_{u, c}^{i} \delta_{u, c}^{j} g\left(x_{i}, x_{j}\right)\right) \\
C_{2}^{\mathrm{SLL}} & =\frac{m_{i} m_{j}}{m_{W}^{2}} \xi_{i s}^{*} V_{i d} \xi_{j s}^{*} V_{j d}\left(f_{4}\left(x_{i}, x_{j}\right)-\delta_{u, c}^{i} \delta_{u, c}^{j} g\left(x_{i}, x_{j}\right)\right) \\
C_{2}^{\mathrm{SRR}} & =\frac{m_{i} m_{j}}{m_{W}^{2}} V_{i s}^{*} \xi_{i d} V_{j s}^{*} \xi_{j d}\left(f_{4}\left(x_{i}, x_{j}\right)-\delta_{u, c}^{i} \delta_{u, c}^{j} g\left(x_{i}, x_{j}\right)\right)
\end{aligned}
$$

where $i=u, c, t$ and $j=u, c, t$ label the internal up-type quark, and a summation over $i$, $j$ is understood. $m_{i}, m_{j}$ are the masses of the internal up-type quarks, and $x_{i}=m_{i}^{2} / m_{W}^{2}$. The loop functions are

$$
\begin{aligned}
f_{1}\left(x_{i}, x_{j}\right)= & -\frac{x_{j}^{2}\left(4-8 x_{j}+x_{j}^{2}\right)}{\left(x_{i}-x_{j}\right)\left(-1+x_{j}\right)^{2}} \log \left(x_{j}\right)+\frac{x_{i}^{2}\left(4-8 x_{i}+x_{i}^{2}\right)}{\left(-1+x_{i}\right)^{2}\left(x_{i}-x_{j}\right)} \log x_{i} \\
& +2-\frac{3}{2}\left(x_{i}+x_{j}\right)-\frac{3\left(x_{i}+x_{j}-x_{i} x_{j}\right)}{\left(1-x_{i}\right)\left(1-x_{j}\right)} \\
f_{2}\left(x_{i}, x_{j}\right)= & -\frac{\left(-4+x_{j}\right)^{2} x_{j}^{2}}{\left(x_{i}-x_{j}\right)\left(x_{j}-1\right)^{2}} \log \left(x_{j}\right)-\frac{\left(-4+x_{i}\right)^{2} x_{i}^{2}}{\left(-1+x_{i}\right)^{2}\left(-x_{i}+x_{j}\right)} \log x_{i} \\
& +14-\frac{3}{2}\left(x_{i}+x_{j}\right)+9 \frac{x_{i}+x_{j}-x_{i} x_{j}}{\left(1-x_{i}\right)\left(1-x_{j}\right)}
\end{aligned}
$$




$$
\begin{aligned}
f_{3}\left(x_{i}, x_{j}\right)= & -\frac{4 x_{j}\left(4-2 x_{j}+x_{j}^{2}\right)}{\left(x_{i}-x_{j}\right)\left(-1+x_{j}\right)^{2}} \log x_{j}+\frac{4 x_{i}\left(4-2 x_{i}+x_{i}^{2}\right)}{\left(-1+x_{i}\right)^{2}\left(x_{i}-x_{j}\right)} \log x_{i}, \\
& +\frac{4\left(2+x_{i}+x_{j}-x_{i} x_{j}\right)}{\left(-1+x_{i}\right)\left(-1+x_{j}\right)}, \\
f_{4}\left(x_{i}, x_{j}\right)= & \frac{2\left(-2+x_{j}\right) x_{j}}{\left(x_{i}-x_{j}\right)\left(-1+x_{j}\right)^{2}} \log x_{j}+\frac{2\left(-2+x_{i}\right) x_{i}}{\left(-1+x_{i}\right)^{2}\left(-x_{i}+x_{j}\right)} \log x_{i} \\
& +\frac{2}{\left(-1+x_{i}\right)\left(-1+x_{j}\right)} .
\end{aligned}
$$

The remaining function, $g\left(x_{i}, x_{j}\right)$, arises from the matching contributions in the theory below $\mu=m_{W}$, which is why it does not receive contributions from diagrams involving the top quark. Up to $\mathcal{O}\left(x_{u}, x_{c}\right)$ corrections, it is given by,

$$
g\left(x_{i}, x_{j}\right)=-4\left(1+\log \frac{\mu^{2}}{m_{W}^{2}}-\frac{x_{i} \log x_{i}-x_{j} \log x_{j}}{x_{i}-x_{j}}\right) .
$$

We verified that the expressions in eq. (A.14) are gauge independent. Our results are in agreement with ref. [215], except that we find matching contributions to $C_{2}^{\mathrm{LR}}, C_{2}^{\mathrm{SLL}}$ and $C_{2}^{\mathrm{SRR}}$, which are not given in ref. [215], and we do not assume unitarity of the $\xi_{i j}$ matrix. Most expressions in eq. (A.14) are UV divergent. For the SM coefficient $C_{1}^{\mathrm{VLL}}$ the unitarity of the CKM guarantees that after summing over $i, j$ the divergence cancels. In all other cases, the divergence indicates mixing of two insertions of RHCC onto $\Delta F=2$ four-fermion operators between the high-energy scale, $\Lambda$, and $m_{W}$. The QCD running of the operators in eq. (A.13) below the scale $m_{W}$ is discussed in detail in ref. [214].

\section{A.3.1 $\quad B-\bar{B}$ oscillations}

The Hamiltonian in eq. (A.12) can be used to compute the mass difference between mass eigenstates in the $B_{d, s}^{0}-\bar{B}_{d, s}^{0}$ systems

$$
\Delta m_{q}=2\left|M_{12}^{(q)}\right|=\frac{\left|\left\langle\bar{B}_{q}^{0}\left|\mathcal{H}^{\mathrm{eff}}\right| B_{q}^{0}\right\rangle\right|}{m_{B_{q}}}=\left(\frac{G_{F}^{2} m_{W}^{2}}{16 \pi^{2}}\right) \frac{1}{m_{B_{q}}}\left|\sum_{i} C_{i}(\mu)\left\langle\bar{B}_{q}^{0}\left|\mathcal{O}_{i}\right| B_{q}^{0}\right\rangle\right| .
$$

The matrix elements for the operators in the basis (A.13) have been computed on the lattice in ref. [216], and we have

$$
\begin{aligned}
\left\langle\bar{B}_{q}^{0}\left|\mathcal{O}_{1}^{\mathrm{VLL}}\right| B_{q}^{0}\right\rangle & =\left\langle\bar{B}_{q}^{0}\left|\mathcal{O}_{1}^{\mathrm{VRR}}\right| B_{q}^{0}\right\rangle=\frac{8}{3} B_{1}^{q}(\mu) m_{B_{q}}^{2} f_{B_{q}}^{2}, \\
\left\langle\bar{B}_{q}^{0}\left|\mathcal{O}_{1}^{\mathrm{SLL}}\right| B_{q}^{0}\right\rangle & =\left\langle\bar{B}_{q}^{0}\left|\mathcal{O}_{1}^{\mathrm{SRR}}\right| B_{q}^{0}\right\rangle=-\frac{5}{12} B_{2}^{q}(\mu) R(\mu) m_{B_{q}}^{2} f_{B_{q}}^{2}, \\
\left\langle\bar{B}_{q}^{0}\left|\mathcal{O}_{2}^{\mathrm{SLL}}\right| B_{q}^{0}\right\rangle & =\left\langle\bar{B}_{q}^{0}\left|\mathcal{O}_{2}^{\mathrm{SRR}}\right| B_{q}^{0}\right\rangle=\left(\frac{5}{3} B_{2}^{q}(\mu)-\frac{2}{3} B_{3}^{q}\right) R(\mu) m_{B_{q}}^{2} f_{B_{q}}^{2}, \\
\left\langle\bar{B}_{q}^{0}\left|\mathcal{O}_{1}^{\mathrm{LR}}\right| B_{q}^{0}\right\rangle & =-\frac{1}{3} B_{5}^{q}(\mu) R(\mu) m_{B_{q}}^{2} f_{B_{q}}^{2}, \\
\left\langle\bar{B}_{q}^{0}\left|\mathcal{O}_{2}^{\mathrm{LR}}\right| B_{q}^{0}\right\rangle & =\frac{1}{2} B_{4}^{q}(\mu) R(\mu) m_{B_{q}}^{2} f_{B_{q}}^{2},
\end{aligned}
$$

where $R(\mu)=m_{B_{q}}^{2} /\left(m_{b}(\mu)+m_{q}(\mu)\right)^{2}$. The bag parameters, in the $\overline{\mathrm{MS}}$ scheme at the scale $\mu=m_{b}=4.2 \mathrm{GeV}$ are summarized in table 13. The FLAG average for the $B_{d}$ and $B_{s}$ 


\begin{tabular}{|c|ccccc|}
\hline & $B_{1}$ & $B_{2}$ & $B_{3}$ & $B_{4}$ & $B_{5}$ \\
\hline$B_{d}^{0}-\bar{B}_{d}^{0}[216]$ & $0.85 \pm 0.04$ & $0.72 \pm 0.03$ & $0.88 \pm 0.13$ & $0.95 \pm 0.05$ & $1.47 \pm 0.12$ \\
$B_{s}^{0}-\bar{B}_{s}^{0}[216]$ & $0.86 \pm 0.03$ & $0.73 \pm 0.03$ & $0.89 \pm 0.12$ & $0.93 \pm 0.04$ & $1.57 \pm 0.11$ \\
$K^{0}-\bar{K}^{0}[93]$ & $0.56 \pm 0.01$ & $0.50 \pm 0.01$ & $0.77 \pm 0.03$ & $0.93 \pm 0.02$ & $0.72 \pm 0.04$ \\
\hline
\end{tabular}

Table 13. Bag parameters for $B_{q}-\bar{B}_{q}$ and $K^{0}-\bar{K}^{0}$ oscillations, in the $\overline{\mathrm{MS}}$ scheme. For $B_{q}-\bar{B}_{q}$ oscillations, we use the results of ref. [216], and the bags parameters are given at the renormalization scale $\mu=m_{b}$. For $K^{0}-\bar{K}^{0}$ oscillation, we quote the FLAG averages of simulations performed with $n_{f}=2+1$ flavors [93]. In this case, $B_{1}=B_{K}$ is given at the renormalization scale $\mu=2 \mathrm{GeV}$, while $B_{2, \ldots, 5}$ are given at $\mu=3 \mathrm{GeV}$.

decay constants is given in table 8. The RGE factors to run the coefficients in eq. (A.14) to the scale $\mu=m_{t}$ to $\mu=m_{b}$ are given in ref. [214].

Neglecting the RHCC contributions which are quadratic in $\xi$, one has to good approximation in the SM,

$$
\Delta m_{q}=2\left|M_{12}^{(q)}\right|=\frac{G_{F}^{2} m_{W}^{2}}{6 \pi^{2}}\left|V_{t q} V_{t b}^{*}\right|^{2} f_{B_{q}}^{2} \hat{B}_{B_{q}} \eta_{B} S_{0}\left(x_{t}, x_{t}\right),
$$

in which $x_{t}$ should be evaluated at $\mu=m_{t}$ and $\eta_{B}=0.55 \pm 0.01$ [217], $S_{0}\left(x_{i}, x_{j}\right)=$ $\frac{1}{4}\left(f_{1}\left(x_{i}, x_{j}\right)-f_{1}\left(0, x_{j}\right)-f_{1}\left(x_{i}, 0\right)+f_{1}(0,0)\right)$. In place of $B_{1}^{s, d}$, it is convenient to introduce the renormalization-group-independent bag parameters $\hat{B}_{B_{d, s}}$, for which we use [93]

$$
f_{B_{d}} \sqrt{\hat{B}_{B_{d}}}=219 \pm 14 \mathrm{MeV}, \quad f_{B_{s}} \sqrt{\hat{B}_{B_{s}}}=270 \pm 16 \mathrm{MeV} .
$$

The experimental values of $\Delta m_{s}$ and $\Delta m_{d}$ are

$$
\Delta m_{d}=(0.5064 \pm 0.0019) \mathrm{ps}^{-1}, \quad \Delta m_{s}=(17.757 \pm 0.021) \mathrm{ps}^{-1} .
$$

\section{A.3.2 $\varepsilon_{K}$}

In the case of $K^{0}-\bar{K}^{0}$ oscillations, the mass difference $\Delta m_{K}=m_{K_{L}}-m_{K_{S}}$ receives sizable long-distance contributions [85], whose uncertainties prevent the use of $\Delta m_{K}$ for a precise extraction of the CKM elements. On the other hand, CPV in $K_{0}-\bar{K}_{0}$ mixing is dominated by short-distance effects. The indirect $\mathrm{CP}$ violation in $K \rightarrow \pi \pi$ decays is parametrized by the parameter $\varepsilon_{K}$, which, up to $\mathcal{O}\left(\xi^{2}\right)$ corrections, is given by [176]

$$
\begin{aligned}
\varepsilon_{K}=\frac{G_{F}^{2} m_{W}^{2}}{12 \pi^{2}} \frac{m_{K} f_{K}^{2} \hat{B}_{K}}{\sqrt{2} \Delta m_{K}} \kappa_{\varepsilon} \operatorname{Im}( & \eta_{c c}\left(V_{c s}^{*} V_{c d}\right)^{2} S_{0}\left(x_{c}\right)+2 \eta_{c t} V_{c s}^{*} V_{c d} V_{t s}^{*} V_{t d} S_{0}\left(x_{c}, x_{t}\right) \\
& \left.+\eta_{t t}\left(V_{t s}^{*} V_{t d}\right)^{2} S_{0}\left(x_{t}\right)\right)
\end{aligned}
$$

Here $x_{t}$ should be evaluated at $\mu=m_{t}$ and $x_{c}$ at $\mu=m_{c}$, furthermore from FLAG and ref. [217]

$$
\begin{aligned}
\hat{B}_{K} & =0.717 \pm 0.018 \pm 0.016, & \kappa_{\varepsilon} & =0.94 \pm 0.02 \\
\eta_{c c} & =1.87 \pm 0.76, & \eta_{c t} & =0.496 \pm 0.047,
\end{aligned}
$$


$\hat{B}_{K}$ is the renormalization-group-invariant bag factor. In ref. [11], we considered long-range contributions to $\varepsilon_{K}$, linear in $\xi_{u s}$ and $\xi_{u d}$. Since the ensuing constraints on these couplings are much weaker than the one from $\epsilon^{\prime} / \epsilon$, we do not include these corrections here.

\section{B Two-loop contributions to the electron EDM}

As the $\xi$ operator only couples the $W$ boson to quarks it mainly induces hadronic EDMs. However, the $\xi_{t b}$ coupling also generates a (fairly small) electron EDM at the two-loop level. Here we briefly describe this contribution.

In the relevant diagram two $W$ bosons connect an electron line with a top-bottom loop which emits a photon. Neglecting the lepton masses, this produces the following contribution to the electron EDM,

$$
\left.v^{2} \tilde{c}_{\gamma l}^{(e e)}\right|_{2 \text { loop }}=-16 N_{c} \frac{y_{t} y_{b}}{(4 \pi)^{4}} \operatorname{Im}\left(\xi_{t b} V_{t b}^{*}\right)\left[\frac{Q_{t}}{Q_{e}} F\left(x_{t}, x_{b}\right)+(t \leftrightarrow b)\right],
$$

where $x_{i} \equiv m_{i}^{2} / m_{W}^{2}$, and

$$
F\left(x_{i}, x_{j}\right)=\frac{1}{2} \int_{0}^{1} d x \frac{x-1}{x^{2}+x\left(x_{j}-x_{i}-1\right)+x_{i}} \ln \frac{x(1-x)}{x\left(x_{j}-x_{i}\right)+x_{i}} .
$$

In the approximation of small $x_{b}$, the loop function becomes,

$$
\begin{aligned}
F\left(x_{t}, 0\right) & =\frac{1}{2}\left[\operatorname{Li}_{2}\left(1-1 / x_{t}\right)-\frac{\pi^{2}}{6}\right], \\
F\left(x_{b}, x_{t}\right) & \simeq \frac{1}{2} \frac{1}{x_{t}-1}\left[\ln x_{t} \ln \frac{x_{b}}{x_{t}}-\left(x_{t}+1\right) \operatorname{Li}_{2}\left(1-1 / x_{t}\right)\right]+\frac{\pi^{2}}{12} .
\end{aligned}
$$

Below the scale $\mu=m_{W}$, a second matching contribution arises from an operator of the form, $\mathcal{L}=C^{(b, e)} \bar{b} \sigma^{\mu \nu} b \bar{e} i \sigma_{\mu \nu} \gamma_{5} e$. This operator is generated at one loop and, in turn, induces the electron EDM through an additional loop. All combined, the matching conditions at the scales $\mu=m_{W}$ and $\mu=m_{b}$ become

$$
\begin{aligned}
v^{2} C^{(b, e)}\left(\mu_{W}\right) & =\frac{y_{t} y_{e}}{(4 \pi)^{2}} \frac{\ln x_{t}}{1-x_{t}} \operatorname{Im}\left(\xi_{t b} V_{t b}^{*}\right), \\
\tilde{c}_{\gamma l}^{(e e)}\left(\mu_{W}\right) & =\left.\tilde{c}_{\gamma l}^{(e e)}\right|_{2 \text { loop }}-\frac{8 N_{c}}{(4 \pi)^{2}} \frac{m_{b} Q_{b}}{m_{e} Q_{e}} \ln \frac{m_{b}^{2}}{\mu_{W}^{2}} C^{(b, e)}\left(\mu_{W}\right), \\
\tilde{c}_{\gamma l}^{(e e)}\left(\mu_{b}^{-}\right) & =\tilde{c}_{\gamma l}^{(e e)}\left(\mu_{b}^{+}\right)+\frac{8 N_{c}}{(4 \pi)^{2}} \frac{m_{b} Q_{b}}{m_{e} Q_{e}} \ln \frac{m_{b}^{2}}{\mu_{b}^{2}} C^{(b, e)}\left(\mu_{b}\right),
\end{aligned}
$$

where $\mu_{W} \simeq m_{W}$ indicates a scale around $\mu=m_{W}$, while $\mu_{b}^{+}\left(\mu_{b}^{-}\right)$refers to a scale just above (below) the $b$-quark threshold. Finally, the RG evolution between $m_{W}$ and $m_{b}$, which determines $C^{(b, e)}\left(\mu_{b}^{+}\right)$and $\tilde{c}_{\gamma l}^{(e e)}\left(\mu_{b}^{+}\right)$, is given by

$$
\frac{d}{d \ln \mu} \tilde{c}_{\gamma l}^{(e e)}(\mu)=16 N_{c} \frac{1}{(4 \pi)^{2}} \frac{m_{b} Q_{b}}{m_{e} Q_{e}} C^{(b, e)}(\mu), \quad \frac{d}{d \ln \mu} C^{(b, e)}(\mu)=2 C_{F} \frac{\alpha_{s}}{4 \pi} C^{(b, e)}(\mu) .
$$

The electron EDM does not evolve under RG $\mu=m_{b}$ (apart from small QED corrections), we use $\tilde{c}_{\gamma l}^{(e e)}(2 \mathrm{GeV})=\tilde{c}_{\gamma l}^{(e e)}\left(\mu_{b}^{-}\right)$which is given in table 7 . 
Open Access. This article is distributed under the terms of the Creative Commons Attribution License (CC-BY 4.0), which permits any use, distribution and reproduction in any medium, provided the original author(s) and source are credited.

\section{References}

[1] R.N. Mohapatra and J.C. Pati, Left-right gauge symmetry and an isoconjugate model of CP-violation, Phys. Rev. D 11 (1975) 566 [INSPIRE].

[2] G. Senjanović and R.N. Mohapatra, Exact left-right symmetry and spontaneous violation of parity, Phys. Rev. D 12 (1975) 1502 [INSPIRE].

[3] G. Senjanović, Spontaneous breakdown of parity in a class of gauge theories, Nucl. Phys. B 153 (1979) 334 [INSPIRE].

[4] R.N. Mohapatra and G. Senjanović, Neutrino mass and spontaneous parity violation, Phys. Rev. Lett. 44 (1980) 912 [inSPIRE].

[5] W. Buchmüller and D. Wyler, Effective Lagrangian analysis of new interactions and flavor conservation, Nucl. Phys. B 268 (1986) 621 [INSPIRE].

[6] B. Grzadkowski, M. Iskrzynski, M. Misiak and J. Rosiek, Dimension-six terms in the Standard Model Lagrangian, JHEP 10 (2010) 085 [arXiv: 1008.4884] [INSPIRE].

[7] Y. Zhang, H. An, X. Ji and R.N. Mohapatra, General CP-violation in minimal left-right symmetric model and constraints on the right-handed scale, Nucl. Phys. B 802 (2008) 247 [arXiv: 0712.4218] [INSPIRE].

[8] A. Maiezza, M. Nemevšek, F. Nesti and G. Senjanović, Left-right symmetry at LHC, Phys. Rev. D 82 (2010) 055022 [arXiv: 1005.5160] [INSPIRE].

[9] G. Senjanović and V. Tello, Right handed quark mixing in left-right symmetric theory, Phys. Rev. Lett. 114 (2015) 071801 [arXiv:1408.3835] [INSPIRE].

[10] G. Senjanović and V. Tello, Restoration of parity and the right-handed analog of the CKM matrix, Phys. Rev. D 94 (2016) 095023 [arXiv: 1502.05704] [InSPIRE].

[11] V. Cirigliano, W. Dekens, J. de Vries and E. Mereghetti, An $\epsilon^{\prime}$ improvement from right-handed currents, Phys. Lett. B 767 (2017) 1 [arXiv:1612.03914] [INSPIRE].

[12] P. Nason, A new method for combining NLO QCD with shower Monte Carlo algorithms, JHEP 11 (2004) 040 [hep-ph/0409146] [INSPIRE].

[13] S. Frixione, P. Nason and C. Oleari, Matching NLO QCD computations with parton shower simulations: the POWHEG method, JHEP 11 (2007) 070 [arXiv: 0709. 2092] [INSPIRE].

[14] S. Alioli, P. Nason, C. Oleari and E. Re, A general framework for implementing NLO calculations in shower Monte Carlo programs: the POWHEG BOX, JHEP 06 (2010) 043 [arXiv: 1002.2581] [INSPIRE].

[15] ATLAS collaboration, Measurement of $W$ boson angular distributions in events with high transverse momentum jets at $\sqrt{s}=8 \mathrm{TeV}$ using the ATLAS detector, Phys. Lett. B 765 (2017) 132 [arXiv: 1609.07045] [INSPIRE].

[16] ATLAS collaboration, Measurement of $W^{ \pm}$and Z-boson production cross sections in $p p$ collisions at $\sqrt{s}=13 \mathrm{TeV}$ with the ATLAS detector, Phys. Lett. B 759 (2016) 601 [arXiv: 1603.09222] [INSPIRE]. 
[17] ATLAS collaboration, Measurement of $k_{\mathrm{T}}$ splitting scales in $W \rightarrow \ell \nu$ events at $\sqrt{s}=7 \mathrm{TeV}$ with the ATLAS detector, Eur. Phys. J. C 73 (2013) 2432 [arXiv:1302.1415] [InSPIRE].

[18] ATLAS collaboration, Measurement of the polarisation of $W$ bosons produced with large transverse momentum in pp collisions at $\sqrt{s}=7 \mathrm{TeV}$ with the ATLAS experiment, Eur. Phys. J. C 72 (2012) 2001 [arXiv:1203.2165] [InSPIRE].

[19] ATLAS collaboration, Measurement of the transverse momentum distribution of $W$ bosons in pp collisions at $\sqrt{s}=7 \mathrm{TeV}$ with the ATLAS detector, Phys. Rev. D 85 (2012) 012005 [arXiv:1108.6308] [INSPIRE].

[20] CMS collaboration, Measurement of the transverse momentum spectra of weak vector bosons produced in proton-proton collisions at $\sqrt{s}=8 \mathrm{TeV}$, JHEP 02 (2017) 096 [arXiv: 1606. 05864] [INSPIRE].

[21] CMS collaboration, Measurement of the differential cross section and charge asymmetry for inclusive $p p \rightarrow W^{ \pm}+X$ production at $\sqrt{s}=8$ TeV, Eur. Phys. J. C 76 (2016) 469 [arXiv: 1603.01803] [INSPIRE].

[22] CMS collaboration, Measurement of inclusive $W$ and $Z$ boson production cross sections in pp collisions at $\sqrt{s}=8$ TeV, Phys. Rev. Lett. 112 (2014) 191802 [arXiv:1402.0923] [INSPIRE].

[23] CMS collaboration, Measurement of the inclusive $W$ and $Z$ production cross sections in $p p$ collisions at $\sqrt{s}=7 \mathrm{TeV}$, JHEP 10 (2011) 132 [arXiv:1107.4789] [INSPIRE].

[24] CMS collaboration, Measurement of the lepton charge asymmetry in inclusive $W$ production in pp collisions at $\sqrt{s}=7 \mathrm{TeV}$, JHEP 04 (2011) 050 [arXiv:1103.3470] [INSPIRE].

[25] CMS collaboration, Measurements of inclusive $W$ and $Z$ cross sections in pp collisions at $\sqrt{s}=7 \mathrm{TeV}$, JHEP 01 (2011) 080 [arXiv:1012.2466] [INSPIRE].

[26] C. Anastasiou, L.J. Dixon, K. Melnikov and F. Petriello, Dilepton rapidity distribution in the Drell-Yan process at NNLO in QCD, Phys. Rev. Lett. 91 (2003) 182002 [hep-ph/0306192] [INSPIRE].

[27] K. Melnikov and F. Petriello, Electroweak gauge boson production at hadron colliders through $O\left(\alpha_{s}^{2}\right)$, Phys. Rev. D 74 (2006) 114017 [hep-ph/0609070] [INSPIRE].

[28] S. Catani, L. Cieri, G. Ferrera, D. de Florian and M. Grazzini, Vector boson production at hadron colliders: a fully exclusive QCD calculation at NNLO, Phys. Rev. Lett. 103 (2009) 082001 [arXiv: 0903.2120] [INSPIRE].

[29] G. Bozzi, S. Catani, G. Ferrera, D. de Florian and M. Grazzini, Production of Drell-Yan lepton pairs in hadron collisions: transverse-momentum resummation at next-to-next-to-leading logarithmic accuracy, Phys. Lett. B 696 (2011) 207 [arXiv: 1007.2351] [INSPIRE].

[30] S. Catani, D. de Florian, G. Ferrera and M. Grazzini, Vector boson production at hadron colliders: transverse-momentum resummation and leptonic decay, JHEP 12 (2015) 047 [arXiv: 1507.06937] [INSPIRE].

[31] A. Karlberg, E. Re and G. Zanderighi, NNLOPS accurate Drell-Yan production, JHEP 09 (2014) 134 [arXiv:1407.2940] [InSPIRE].

[32] S. Alioli et al., Precision studies of observables in $p p \rightarrow W \rightarrow \ell \nu$ and $p p \rightarrow \gamma, Z \rightarrow \ell^{+} \ell^{-}$ processes at the LHC, submitted to Working Group Report (2016) [arXiv:1606. 02330] [INSPIRE]. 
[33] S. Alioli, P. Nason, C. Oleari and E. Re, NLO vector-boson production matched with shower in POWHEG, JHEP 07 (2008) 060 [arXiv:0805.4802] [INSPIRE].

[34] R.K. Ellis, W.J. Stirling and B.R. Webber, QCD and collider physics, Camb. Monogr. Part. Phys. Nucl. Phys. Cosmol. 8 (1996) 1 [InSPIRE].

[35] Z. Bern et al., Left-handed W bosons at the LHC, Phys. Rev. D 84 (2011) 034008 [arXiv:1103.5445] [INSPIRE].

[36] G. Ferrera, M. Grazzini and F. Tramontano, Associated WH production at hadron colliders: a fully exclusive QCD calculation at NNLO, Phys. Rev. Lett. 107 (2011) 152003 [arXiv:1107.1164] [INSPIRE].

[37] G. Ferrera, M. Grazzini and F. Tramontano, Higher-order QCD effects for associated $W H$ production and decay at the LHC, JHEP 04 (2014) 039 [arXiv:1312.1669] [InSPIRE].

[38] J.M. Campbell, R.K. Ellis and C. Williams, Associated production of a Higgs boson at NNLO, JHEP 06 (2016) 179 [arXiv:1601.00658] [inSPIRE].

[39] W. Astill, W. Bizon, E. Re and G. Zanderighi, NNLOPS accurate associated HW production, JHEP 06 (2016) 154 [arXiv: 1603.01620] [INSPIRE].

[40] G. Luisoni, P. Nason, C. Oleari and F. Tramontano, $H W^{ \pm} / H Z+0$ and 1 jet at $N L O$ with the POWHEG BOX interfaced to GoSam and their merging within MiNLO, JHEP 10 (2013) 083 [arXiv: 1306. 2542] [inSPIRE].

[41] ATLAS and CMS collaborations, Measurements of the Higgs boson production and decay rates and constraints on its couplings from a combined ATLAS and CMS analysis of the LHC pp collision data at $\sqrt{s}=7$ and 8 TeV, JHEP 08 (2016) 045 [arXiv:1606.02266] [INSPIRE].

[42] E.E. Jenkins, A.V. Manohar and M. Trott, Renormalization group evolution of the Standard Model dimension six operators II: Yukawa dependence, JHEP 01 (2014) 035 [arXiv:1310.4838] [INSPIRE].

[43] R. Alonso, E.E. Jenkins, A.V. Manohar and M. Trott, Renormalization group evolution of the Standard Model dimension six operators III: gauge coupling dependence and phenomenology, JHEP 04 (2014) 159 [arXiv:1312.2014] [INSPIRE].

[44] ATLAS collaboration, Search for the Standard Model Higgs boson produced in association with a vector boson and decaying to a b $\bar{b}$ pair in pp collisions at $13 \mathrm{TeV}$ using the ATLAS detector, ATLAS-CONF-2016-091, CERN, Geneva Switzerland, (2016).

[45] F.A. Dreyer and A. Karlberg, Vector-boson fusion Higgs production at three loops in QCD, Phys. Rev. Lett. 117 (2016) 072001 [arXiv:1606.00840] [INSPIRE].

[46] M. Cacciari, F.A. Dreyer, A. Karlberg, G.P. Salam and G. Zanderighi, Fully differential vector-boson-fusion Higgs production at next-to-next-to-leading order, Phys. Rev. Lett. 115 (2015) 082002 [arXiv: 1506. 02660] [inSPIRE].

[47] P. Nason and C. Oleari, NLO Higgs boson production via vector-boson fusion matched with shower in POWHEG, JHEP 02 (2010) 037 [arXiv:0911.5299] [INSPIRE].

[48] ATLAS collaboration, Comprehensive measurements of t-channel single top-quark production cross sections at $\sqrt{s}=7 \mathrm{TeV}$ with the ATLAS detector, Phys. Rev. D 90 (2014) 112006 [arXiv: 1406.7844] [INSPIRE]. 
[49] CMS collaboration, Measurement of the single-top-quark t-channel cross section in pp collisions at $\sqrt{s}=7 \mathrm{TeV}$, JHEP 12 (2012) 035 [arXiv:1209.4533] [INSPIRE].

[50] ATLAS collaboration, Fiducial, total and differential cross-section measurements of t-channel single top-quark production in pp collisions at $8 \mathrm{TeV}$ using data collected by the ATLAS detector, arXiv:1702.02859 [INSPIRE].

[51] CMS collaboration, Measurement of the t-channel single-top-quark production cross section and of the $\left|V_{t b}\right|$ CKM matrix element in pp collisions at $\sqrt{s}=8 \mathrm{TeV}$, JHEP 06 (2014) 090 [arXiv: 1403.7366] [INSPIRE].

[52] ATLAS collaboration, Measurement of the inclusive cross-sections of single top-quark and top-antiquark $t$-channel production in pp collisions at $\sqrt{s}=13 \mathrm{TeV}$ with the ATLAS detector, JHEP 04 (2017) 086 [arXiv: 1609.03920] [INSPIRE].

[53] CMS collaboration, Cross section measurement of $t$-channel single top quark production in pp collisions at $\sqrt{s}=13 \mathrm{TeV}$, submitted to Phys. Lett. B (2016) [arXiv:1610.00678] [INSPIRE].

[54] ATLAS collaboration, Measurement of the production cross-section of a single top quark in association with a $W$ boson at $8 \mathrm{TeV}$ with the ATLAS experiment, JHEP 01 (2016) 064 [arXiv: 1510.03752] [INSPIRE].

[55] CMS collaboration, Observation of the associated production of a single top quark and a $W$ boson in pp collisions at $\sqrt{s}=8$ TeV, Phys. Rev. Lett. 112 (2014) 231802 [arXiv: 1401.2942] [INSPIRE].

[56] CDF and D0 collaborations, T.A. Aaltonen et al., Observation of s-channel production of single top quarks at the Tevatron, Phys. Rev. Lett. 112 (2014) 231803 [arXiv:1402.5126] [INSPIRE].

[57] M. Brucherseifer, F. Caola and K. Melnikov, On the NNLO QCD corrections to single-top production at the LHC, Phys. Lett. B 736 (2014) 58 [arXiv:1404.7116] [INSPIRE].

[58] M. Brucherseifer, F. Caola and K. Melnikov, $O\left(\alpha_{s}^{2}\right)$ corrections to fully-differential top quark decays, JHEP 04 (2013) 059 [arXiv:1301.7133] [INSPIRE].

[59] J. Gao, C.S. Li and H.X. Zhu, Top quark decay at next-to-next-to leading order in QCD, Phys. Rev. Lett. 110 (2013) 042001 [arXiv: 1210.2808] [INSPIRE].

[60] E.L. Berger, J. Gao, C.P. Yuan and H.X. Zhu, NNLO QCD corrections to t-channel single top-quark production and decay, Phys. Rev. D 94 (2016) 071501 [arXiv:1606.08463] [INSPIRE].

[61] J.M. Campbell, R. Frederix, F. Maltoni and F. Tramontano, Next-to-leading-order predictions for t-channel single-top production at hadron colliders, Phys. Rev. Lett. 102 (2009) 182003 [arXiv:0903.0005] [INSPIRE].

[62] R. Frederix, E. Re and P. Torrielli, Single-top t-channel hadroproduction in the four-flavour scheme with POWHEG and aMC@NLO, JHEP 09 (2012) 130 [arXiv:1207.5391] [INSPIRE].

[63] T. Ježo and P. Nason, On the treatment of resonances in next-to-leading order calculations matched to a parton shower, JHEP 12 (2015) 065 [arXiv: 1509.09071] [INSPIRE].

[64] A.S. Papanastasiou, R. Frederix, S. Frixione, V. Hirschi and F. Maltoni, Single-top t-channel production with off-shell and non-resonant effects, Phys. Lett. B 726 (2013) 223 [arXiv: 1305.7088] [INSPIRE]. 
[65] R. Frederix, S. Frixione, A.S. Papanastasiou, S. Prestel and P. Torrielli, Off-shell single-top production at NLO matched to parton showers, JHEP 06 (2016) 027 [arXiv: 1603.01178] [INSPIRE].

[66] S. Alioli, P. Nason, C. Oleari and E. Re, NLO single-top production matched with shower in POWHEG: s- and t-channel contributions, JHEP 09 (2009) 111 [Erratum ibid. 02 (2010) 011] [arXiv:0907.4076] [INSPIRE].

[67] S. Alekhin et al., The PDF4LHC working group interim report, arXiv:1101.0536 [INSPIRE].

[68] H.-L. Lai et al., New parton distributions for collider physics, Phys. Rev. D 82 (2010) 074024 [arXiv: 1007.2241] [INSPIRE].

[69] A.D. Martin, W.J. Stirling, R.S. Thorne and G. Watt, Parton distributions for the LHC, Eur. Phys. J. C 63 (2009) 189 [arXiv:0901.0002] [INSPIRE].

[70] R.D. Ball et al., Parton distributions with LHC data, Nucl. Phys. B 867 (2013) 244 [arXiv: 1207.1303] [INSPIRE].

[71] Particle Data Group collaboration, C. Patrignani et al., Review of particle physics, Chin. Phys. C 40 (2016) 100001 [inSPIRE].

[72] CDF and D0 collaboration, T. Aaltonen et al., Combination of CDF and D0 measurements of the $W$ boson helicity in top quark decays, Phys. Rev. D 85 (2012) 071106 [arXiv:1202.5272] [INSPIRE].

[73] ATLAS collaboration, Measurement of the $W$ boson polarization in top quark decays with the ATLAS detector, JHEP 06 (2012) 088 [arXiv:1205.2484] [INSPIRE].

[74] CMS collaboration, Measurement of the $W$-boson helicity in top-quark decays from $t \bar{t}$ production in lepton+jets events in pp collisions at $\sqrt{s}=7 \mathrm{TeV}$, JHEP 10 (2013) 167 [arXiv: 1308.3879] [INSPIRE].

[75] ATLAS collaboration, Search for anomalous couplings in the Wtb vertex from the measurement of double differential angular decay rates of single top quarks produced in the t-channel with the ATLAS detector, JHEP 04 (2016) 023 [arXiv: 1510.03764] [INSPIRE].

[76] CMS collaboration, Measurement of the $W$ boson helicity in events with a single reconstructed top quark in pp collisions at $\sqrt{s}=8 \mathrm{TeV}$, JHEP 01 (2015) 053 [arXiv:1410.1154] [INSPIRE].

[77] ATLAS collaboration, Measurement of the $W$ boson polarisation in $t \bar{t}$ events from $p p$ collisions at $\sqrt{s}=8 \mathrm{TeV}$ in the lepton+jets channel with ATLAS, arXiv:1612.02577 [INSPIRE].

[78] A. Czarnecki, J.G. Korner and J.H. Piclum, Helicity fractions of $W$ bosons from top quark decays at NNLO in QCD, Phys. Rev. D 81 (2010) 111503 [arXiv:1005.2625] [INSPIRE].

[79] J. Drobnak, S. Fajfer and J.F. Kamenik, New physics in $t \rightarrow b W$ decay at next-to-leading order in QCD, Phys. Rev. D 82 (2010) 114008 [arXiv: 1010.2402] [INSPIRE].

[80] E.E. Jenkins, A.V. Manohar and M. Trott, Renormalization group evolution of the Standard Model dimension six operators I: formalism and $\lambda$ dependence, JHEP 10 (2013) 087 [arXiv: 1308.2627] [INSPIRE].

[81] M. Spira, QCD effects in Higgs physics, Fortsch. Phys. 46 (1998) 203 [hep-ph/9705337] [INSPIRE]. 
[82] G. Perez, Y. Soreq, E. Stamou and K. Tobioka, Constraining the charm Yukawa and Higgs-quark coupling universality, Phys. Rev. D 92 (2015) 033016 [arXiv:1503.00290] [INSPIRE].

[83] Y. Soreq, H.X. Zhu and J. Zupan, Light quark Yukawa couplings from Higgs kinematics, JHEP 12 (2016) 045 [arXiv:1606.09621] [INSPIRE].

[84] C. Bobeth, A.J. Buras, A. Celis and M. Jung, Yukawa enhancement of Z-mediated new physics in $\Delta S=2$ and $\Delta B=2$ processes, arXiv: 1703.04753 [INSPIRE].

[85] G. Buchalla, A.J. Buras and M.E. Lautenbacher, Weak decays beyond leading logarithms, Rev. Mod. Phys. 68 (1996) 1125 [hep-ph/9512380] [INSPIRE].

[86] W. Dekens and J. de Vries, Renormalization group running of dimension-six sources of parity and time-reversal violation, JHEP 05 (2013) 149 [arXiv:1303.3156] [INSPIRE].

[87] S. Weinberg, Larger Higgs exchange terms in the neutron electric dipole moment, Phys. Rev. Lett. 63 (1989) 2333 [INSPIRE].

[88] F. Wilczek and A. Zee, $\Delta I=1 / 2$ rule and right-handed currents: heavy quark expansion and limitation on Zweig's rule, Phys. Rev. D 15 (1977) 2660 [INSPIRE].

[89] E. Braaten, C.-S. Li and T.-C. Yuan, The evolution of Weinberg's gluonic CP violation operator, Phys. Rev. Lett. 64 (1990) 1709 [INSPIRE].

[90] P.L. Cho and M. Misiak, $b \rightarrow$ s $\gamma$ decay in $\mathrm{SU}(2)_{L} \times \mathrm{SU}(2)_{R} \times \mathrm{U}(1)$ extensions of the Standard Model, Phys. Rev. D 49 (1994) 5894 [hep-ph/9310332] [INSPIRE].

[91] M. Misiak, private communication, (2016).

[92] D. Chang, C.S. Li and T.C. Yuan, Larger neutron electric dipole moment in left-right symmetric models, Phys. Rev. D 42 (1990) 867 [InSPIRE].

[93] S. Aoki et al., Review of lattice results concerning low-energy particle physics, Eur. Phys. J. C 77 (2017) 112 [arXiv: 1607.00299] [InSPIRE].

[94] J.C. Hardy and I.S. Towner, Superallowed $0^{+} \rightarrow 0^{+}$nuclear $\beta$ decays: 2014 critical survey, with precise results for $V_{u d}$ and CKM unitarity, Phys. Rev. C 91 (2015) 025501 [arXiv:1411.5987] [INSPIRE].

[95] S. Aoki et al., Review of lattice results concerning low-energy particle physics, Eur. Phys. J. C 74 (2014) 2890 [arXiv:1310.8555] [InSPIRE].

[96] Flavianet Working Group on Kaon Decays collaboration, M. Antonelli et al., An evaluation of $\left|V_{u s}\right|$ and precise tests of the Standard Model from world data on leptonic and semileptonic kaon decays, Eur. Phys. J. C 69 (2010) 399 [arXiv:1005.2323] [InSPIRE].

[97] H.P. Mumm et al., A new limit on time-reversal violation in beta decay, Phys. Rev. Lett. 107 (2011) 102301 [arXiv:1104.2778] [INSPIRE].

[98] K.K. Vos, H.W. Wilschut and R.G.E. Timmermans, Symmetry violations in nuclear and neutron beta decay, Rev. Mod. Phys. 87 (2015) 1483 [arXiv:1509.04007] [InSPIRE].

[99] J.D. Jackson, S.B. Treiman and H.W. Wyld, Possible tests of time reversal invariance in beta decay, Phys. Rev. 106 (1957) 517 [INSPIRE].

[100] A.V. Manohar and M.B. Wise, Heavy quark physics, Camb. Monogr. Part. Phys. Nucl. Phys. Cosmol. 10 (2000) 1 [InSPIRE]. 
[101] Y. Amhis et al., Averages of b-hadron, c-hadron and $\tau$-lepton properties as of summer 2016, arXiv: 1612.07233 [INSPIRE].

[102] D. Becirevic, S. Fajfer, I. Nisandzic and A. Tayduganov, Angular distributions of $\bar{B} \rightarrow D^{(*)} \ell \bar{\nu}_{\ell}$ decays and search of new physics, arXiv:1602.03030 [INSPIRE].

[103] C.W. Bauer, Z. Ligeti, M. Luke and A.V. Manohar, B decay shape variables and the precision determination of $\left|V_{c b}\right|$ and $m_{b}$, Phys. Rev. D 67 (2003) 054012 [hep-ph/0210027] [INSPIRE].

[104] P. Gambino, B semileptonic moments at NNLO, JHEP 09 (2011) 055 [arXiv:1107.3100] [INSPIRE].

[105] P. Gambino and C. Schwanda, Inclusive semileptonic fits, heavy quark masses and $V_{c b}$, Phys. Rev. D 89 (2014) 014022 [arXiv:1307.4551] [INSPIRE].

[106] A. Alberti, P. Gambino, K.J. Healey and S. Nandi, Precision determination of the Cabibbo-Kobayashi-Maskawa element $V_{c b}$, Phys. Rev. Lett. 114 (2015) 061802 [arXiv:1411.6560] [INSPIRE].

[107] B. Dassinger, R. Feger and T. Mannel, Complete Michel parameter analysis of inclusive semileptonic $b \rightarrow c$ transition, Phys. Rev. D 79 (2009) 075015 [arXiv:0803.3561] [INSPIRE].

[108] R. Feger, T. Mannel, V. Klose, H. Lacker and T. Luck, Limit on a right-handed admixture to the weak $b \rightarrow c$ current from semileptonic decays, Phys. Rev. D 82 (2010) 073002 [arXiv: 1003.4022] [INSPIRE].

[109] A. Crivellin and S. Pokorski, Can the differences in the determinations of $V_{u b}$ and $V_{c b}$ be explained by new physics?, Phys. Rev. Lett. 114 (2015) 011802 [arXiv:1407.1320] [INSPIRE].

[110] F.U. Bernlochner, Z. Ligeti and S. Turczyk, New ways to search for right-handed current in $B \rightarrow \rho \ell \bar{\nu}$ decay, Phys. Rev. D 90 (2014) 094003 [arXiv:1408.2516] [InSPIRE].

[111] C.W. Bauer, Z. Ligeti and M.E. Luke, Precision determination of $\left|V_{u b}\right|$ from inclusive decays, Phys. Rev. D 64 (2001) 113004 [hep-ph/0107074] [INSPIRE].

[112] B.O. Lange, M. Neubert and G. Paz, Theory of charmless inclusive B decays and the extraction of $V_{u b}$, Phys. Rev. D 72 (2005) 073006 [hep-ph/0504071] [InSPIRE].

[113] W. Detmold, C. Lehner and S. Meinel, $\Lambda_{b} \rightarrow p \ell^{-} \bar{\nu}_{\ell}$ and $\Lambda_{b} \rightarrow \Lambda_{c} \ell^{-} \bar{\nu}_{\ell}$ form factors from lattice QCD with relativistic heavy quarks, Phys. Rev. D 92 (2015) 034503 [arXiv: 1503.01421] [INSPIRE].

[114] LHCb collaboration, Determination of the quark coupling strength $\left|V_{u b}\right|$ using baryonic decays, Nature Phys. 11 (2015) 743 [arXiv:1504.01568] [INSPIRE].

[115] A.V. Manohar and M.B. Wise, Inclusive semileptonic $B$ and polarized $\Lambda_{b}$ decays from QCD, Phys. Rev. D 49 (1994) 1310 [hep-ph/9308246] [InSPIRE].

[116] ACME collaboration, J. Baron et al., Order of magnitude smaller limit on the electric dipole moment of the electron, Science 343 (2014) 269 [arXiv:1310.7534] [INSPIRE].

[117] C.A. Baker et al., An improved experimental limit on the electric dipole moment of the neutron, Phys. Rev. Lett. 97 (2006) 131801 [hep-ex/0602020] [INSPIRE].

[118] J.M. Pendlebury et al., Revised experimental upper limit on the electric dipole moment of the neutron, Phys. Rev. D 92 (2015) 092003 [arXiv:1509.04411] [INSPIRE]. 
[119] W.C. Griffith, M.D. Swallows, T.H. Loftus, M.V. Romalis, B.R. Heckel and E.N. Fortson, Improved limit on the permanent electric dipole moment of ${ }^{199} \mathrm{Hg}$, Phys. Rev. Lett. 102 (2009) 101601 [INSPIRE].

[120] B. Graner, Y. Chen, E.G. Lindahl and B.R. Heckel, Reduced limit on the permanent electric dipole moment of ${ }^{199} \mathrm{Hg}$, Phys. Rev. Lett. 116 (2016) 161601 [arXiv:1601.04339] [InSPIRE].

[121] M.A. Rosenberry and T.E. Chupp, Atomic electric dipole moment measurement using spin exchange pumped masers of ${ }^{129}$ Xe and ${ }^{3}$ He, Phys. Rev. Lett. 86 (2001) 22.

[122] M. Bishof et al., Improved limit on the ${ }^{225}$ Ra electric dipole moment, Phys. Rev. C 94 (2016) 025501 [arXiv: 1606.04931] [INSPIRE].

[123] R.H. Parker et al., First measurement of the atomic electric dipole moment of ${ }^{225}$ Ra, Phys. Rev. Lett. 114 (2015) 233002 [arXiv:1504.07477] [INSPIRE].

[124] K. Kumar, Z.-T. Lu and M.J. Ramsey-Musolf, Working group report: nucleons, nuclei and atoms, in Community Summer Study 2013: Snowmass on the Mississippi (CSS2013), Minneapolis MN U.S.A., 29 July-6 August 2013 [arXiv:1312.5416] [INSPIRE].

[125] T. Chupp and M. Ramsey-Musolf, Electric dipole moments: a global analysis, Phys. Rev. C 91 (2015) 035502 [arXiv:1407.1064] [INSPIRE].

[126] J. de Vries, E. Mereghetti, R.G.E. Timmermans and U. van Kolck, The effective chiral Lagrangian from dimension-six parity and time-reversal violation, Annals Phys. 338 (2013) 50 [arXiv: 1212.0990] [INSPIRE].

[127] J. Bsaisou, U.-G. Meißner, A. Nogga and A. Wirzba, P- and T-violating Lagrangians in chiral effective field theory and nuclear electric dipole moments, Annals Phys. 359 (2015) 317 [arXiv: 1412.5471] [INSPIRE].

[128] T. Blum et al., Lattice determination of the $K \rightarrow(\pi \pi)_{I=2}$ decay amplitude $A_{2}$, Phys. Rev. D 86 (2012) 074513 [arXiv:1206.5142] [INSPIRE].

[129] T. Blum et al., $K \rightarrow \pi \pi \Delta I=3 / 2$ decay amplitude in the continuum limit, Phys. Rev. D 91 (2015) 074502 [arXiv: 1502.00263] [INSPIRE].

[130] J. de Vries, E. Mereghetti, C.-Y. Seng and A. Walker-Loud, Lattice QCD spectroscopy for hadronic CP-violation, Phys. Lett. B 766 (2017) 254 [arXiv: 1612.01567] [INSPIRE].

[131] M. Pospelov and A. Ritz, Neutron EDM from electric and chromoelectric dipole moments of quarks, Phys. Rev. D 63 (2001) 073015 [hep-ph/0010037] [InSPIRE].

[132] O. Lebedev, K.A. Olive, M. Pospelov and A. Ritz, Probing CP-violation with the deuteron electric dipole moment, Phys. Rev. D 70 (2004) 016003 [hep-ph/0402023] [INSPIRE].

[133] M. Pospelov and A. Ritz, Electric dipole moments as probes of new physics, Annals Phys. 318 (2005) 119 [hep-ph/0504231] [INSPIRE].

[134] J. Hisano, J.Y. Lee, N. Nagata and Y. Shimizu, Reevaluation of neutron electric dipole moment with QCD sum rules, Phys. Rev. D 85 (2012) 114044 [arXiv:1204.2653] [INSPIRE].

[135] R.D. Peccei and H.R. Quinn, CP conservation in the presence of instantons, Phys. Rev. Lett. 38 (1977) 1440 [INSPIRE].

[136] C.-Y. Seng, J. de Vries, E. Mereghetti, H.H. Patel and M. Ramsey-Musolf, Nucleon electric dipole moments and the isovector parity- and time-reversal-odd pion-nucleon coupling, Phys. Lett. B 736 (2014) 147 [arXiv:1401.5366] [InSPIRE]. 
[137] A. Maiezza and M. Nemevšek, Strong $P$ invariance, neutron electric dipole moment and minimal left-right parity at LHC, Phys. Rev. D 90 (2014) 095002 [arXiv:1407.3678] [INSPIRE].

[138] T. Bhattacharya, V. Cirigliano, R. Gupta, H.-W. Lin and B. Yoon, Neutron electric dipole moment and tensor charges from lattice QCD, Phys. Rev. Lett. 115 (2015) 212002 [arXiv: 1506. 04196] [INSPIRE].

[139] PNDME collaboration, T. Bhattacharya et al., Iso-vector and iso-scalar tensor charges of the nucleon from lattice QCD, Phys. Rev. D 92 (2015) 094511 [arXiv:1506.06411] [INSPIRE].

[140] T. Bhattacharya, V. Cirigliano, S. Cohen, R. Gupta, H.-W. Lin and B. Yoon, Axial, scalar and tensor charges of the nucleon from $2+1+1$-flavor lattice QCD, Phys. Rev. D 94 (2016) 054508 [arXiv: 1606.07049] [INSPIRE].

[141] T. Bhattacharya, V. Cirigliano, R. Gupta, E. Mereghetti and B. Yoon, Neutron electric dipole moment from quark chromoelectric dipole moment, PoS (LATTICE 2015) 238 [arXiv: 1601.02264] [INSPIRE].

[142] T. Bhattacharya, V. Cirigliano, R. Gupta and B. Yoon, Quark chromoelectric dipole moment contribution to the neutron electric dipole moment, in Proceedings, $34^{\text {th }}$ International Symposium on Lattice Field Theory (Lattice 2016), Southampton U.K., 24-30 July 2016 [PoS(LATTICE2016) 225] [arXiv: 1612.08438] [INSPIRE].

[143] M. Abramczyk, S. Aoki, T. Blum, T. Izubuchi, H. Ohki and S. Syritsyn, On lattice calculation of electric dipole moments and form factors of the nucleon, arXiv:1701.07792 [INSPIRE].

[144] D.A. Demir, M. Pospelov and A. Ritz, Hadronic EDMs, the Weinberg operator and light gluinos, Phys. Rev. D 67 (2003) 015007 [hep-ph/0208257] [INSPIRE].

[145] J.H. de Jesus and J. Engel, Time-reversal-violating Schiff moment of ${ }^{199} \mathrm{Hg}$, Phys. Rev. C 72 (2005) 045503 [nucl-th/0507031] [INSPIRE].

[146] J. Dobaczewski and J. Engel, Nuclear time-reversal violation and the Schiff moment of ${ }^{225}$ Ra, Phys. Rev. Lett. 94 (2005) 232502 [nucl-th/0503057] [inSPIRE].

[147] S. Ban, J. Dobaczewski, J. Engel and A. Shukla, Fully self-consistent calculations of nuclear Schiff moments, Phys. Rev. C 82 (2010) 015501 [arXiv:1003.2598] [INSPIRE].

[148] V.A. Dzuba, V.V. Flambaum and S.G. Porsev, Calculation of $(P, T)$-odd electric dipole moments for diamagnetic atoms ${ }^{129} \mathrm{Xe},{ }^{171} \mathrm{Yb},{ }^{199} \mathrm{Hg},{ }^{211} \mathrm{Rn}$ and ${ }^{225} \mathrm{Ra}$, Phys. Rev. A 80 (2009) 032120 [arXiv:0906.5437] [INSPIRE].

[149] J. Engel, M.J. Ramsey-Musolf and U. van Kolck, Electric dipole moments of nucleons, nuclei and atoms: the Standard Model and beyond, Prog. Part. Nucl. Phys. 71 (2013) 21 [arXiv: 1303.2371] [INSPIRE].

[150] J. de Vries et al., Electric dipole moments of light nuclei from chiral effective field theory, Phys. Rev. C 84 (2011) 065501 [arXiv:1109.3604] [INSPIRE].

[151] Y. Singh and B.K. Sahoo, Rigorous limits for hadronic and semi-leptonic CP-violating coupling constants from the electric dipole moment of ${ }^{199} \mathrm{Hg}$, Phys. Rev. A 91 (2015) 030501 [arXiv: 1408.4337] [INSPIRE]. 
[152] Y. Singh and B.K. Sahoo, Electric dipole moment of ${ }^{225} R a$ due to P- and T-violating weak interactions, Phys. Rev. A 92 (2015) 022502 [arXiv:1504.00269] [INSPIRE].

[153] N. Yamanaka and E. Hiyama, Enhancement of the CP-odd effect in the nuclear electric dipole moment of ${ }^{6}$ Li, Phys. Rev. C 91 (2015) 054005 [arXiv: 1503.04446] [INSPIRE].

[154] N. Yamanaka, B.K. Sahoo, N. Yoshinaga, T. Sato, K. Asahi and B.P. Das, Probing exotic phenomena at the interface of nuclear and particle physics with the electric dipole moments of diamagnetic atoms: a unique window to hadronic and semi-leptonic CP-violation, Eur. Phys. J. A 53 (2017) 54 [arXiv:1703.01570] [inSPIRE].

[155] N. Yoshinaga, K. Higashiyama and R. Arai, Shell model estimate of nuclear electric dipole moments, Prog. Theor. Phys. 124 (2010) 1115.

[156] CKMfitTer Group collaboration, J. Charles et al., CP violation and the CKM matrix: assessing the impact of the asymmetric B factories, Eur. Phys. J. C 41 (2005) 1 [hep-ph/0406184] [INSPIRE].

[157] M. Ciuchini, E. Franco, G. Martinelli and L. Reina, $\epsilon^{\prime} / \epsilon$ at the next-to-leading order in QCD and QED, Phys. Lett. B 301 (1993) 263 [hep-ph/9212203] [INSPIRE].

[158] A.J. Buras, M. Jamin and M.E. Lautenbacher, The anatomy of $\epsilon^{\prime} / \epsilon$ beyond leading logarithms with improved hadronic matrix elements, Nucl. Phys. B 408 (1993) 209 [hep-ph/9303284] [INSPIRE].

[159] M. Ciuchini, E. Franco, G. Martinelli, L. Reina and L. Silvestrini, An upgraded analysis of $\epsilon^{\prime} / \epsilon$ at the next-to-leading order, Z. Phys. C 68 (1995) 239 [hep-ph/9501265] [INSPIRE].

[160] RBC and UKQCD collaborations, Z. Bai et al., Standard Model prediction for direct CP-violation in $K \rightarrow \pi \pi$ decay, Phys. Rev. Lett. 115 (2015) 212001 [arXiv:1505.07863] [INSPIRE].

[161] P. Chen, H. Ke and X. Ji, Direct CP-violation in K-decay and minimal left-right symmetry scale, Phys. Lett. B 677 (2009) 157 [arXiv:0810.2576] [INSPIRE].

[162] S. Bertolini, A. Maiezza and F. Nesti, $K \rightarrow \pi \pi$ hadronic matrix elements of left-right current-current operators, Phys. Rev. D 88 (2013) 034014 [arXiv: 1305.5739] [INSPIRE].

[163] I. Baum, V. Lubicz, G. Martinelli, L. Orifici and S. Simula, Matrix elements of the electromagnetic operator between kaon and pion states, Phys. Rev. D 84 (2011) 074503 [arXiv:1108.1021] [INSPIRE].

[164] T. Hurth, E. Lunghi and W. Porod, Untagged $\bar{B} \rightarrow X_{s+d} \gamma C P$ asymmetry as a probe for new physics, Nucl. Phys. B 704 (2005) 56 [hep-ph/0312260] [INSPIRE].

[165] M. Misiak et al., Estimate of $B\left(\bar{B} \rightarrow X_{s} \gamma\right)$ at $O\left(\alpha_{s}^{2}\right)$, Phys. Rev. Lett. 98 (2007) 022002 [hep-ph/0609232] [INSPIRE].

[166] M. Misiak et al., Updated NNLO QCD predictions for the weak radiative B-meson decays, Phys. Rev. Lett. 114 (2015) 221801 [arXiv:1503.01789] [INSPIRE].

[167] M. Czakon, P. Fiedler, T. Huber, M. Misiak, T. Schutzmeier and M. Steinhauser, The $\left(Q_{7}, Q_{1,2}\right)$ contribution to $\bar{B} \rightarrow X_{s} \gamma$ at $O\left(\alpha_{s}^{2}\right)$, JHEP 04 (2015) 168 [arXiv:1503.01791] [INSPIRE].

[168] W. Altmannshofer and D.M. Straub, Cornering new physics in $b \rightarrow s$ transitions, JHEP 08 (2012) 121 [arXiv: 1206.0273] [INSPIRE]. 
[169] W. Altmannshofer, P. Paradisi and D.M. Straub, Model-independent constraints on new physics in $b \rightarrow s$ transitions, JHEP 04 (2012) 008 [arXiv:1111.1257] [INSPIRE].

[170] M. Benzke, S.J. Lee, M. Neubert and G. Paz, Long-distance dominance of the CP asymmetry in $B \rightarrow X_{s, d}+\gamma$ decays, Phys. Rev. Lett. 106 (2011) 141801 [arXiv:1012.3167] [INSPIRE].

[171] A. Paul and D.M. Straub, Constraints on new physics from radiative B decays, JHEP 04 (2017) 027 [arXiv: 1608.02556] [INSPIRE].

[172] P. Ball and R. Zwicky, Time-dependent CP asymmetry in $B \rightarrow K^{*} \gamma$ as a (quasi) null test of the Standard Model, Phys. Lett. B 642 (2006) 478 [hep-ph/0609037] [INSPIRE].

[173] P. Ball, G.W. Jones and R. Zwicky, $B \rightarrow V \gamma$ beyond QCD factorisation, Phys. Rev. D 75 (2007) 054004 [hep-ph/0612081] [INSPIRE].

[174] A.J. Buras and R. Fleischer, Quark mixing, CP-violation and rare decays after the top quark discovery, Adv. Ser. Direct. High Energy Phys. 15 (1998) 65 [hep-ph/9704376] [INSPIRE].

[175] M. Artuso, G. Borissov and A. Lenz, CP violation in the $B_{s}^{0}$ system, Rev. Mod. Phys. 88 (2016) 045002 [arXiv: 1511.09466] [inSPIRE].

[176] A.J. Buras, Weak Hamiltonian, CP-violation and rare decays, in Probing the Standard Model of particle interactions. Proceedings, Summer School in Theoretical Physics, NATO Advanced Study Institute, $68^{\text {th }}$ session, Les Houches France, 28 July-5 September 1997 [hep-ph/9806471] [INSPIRE].

[177] A.J. Buras, M. Gorbahn, S. Jäger and M. Jamin, Improved anatomy of $\epsilon^{\prime} / \epsilon$ in the Standard Model, JHEP 11 (2015) 202 [arXiv:1507.06345] [INSPIRE].

[178] A.J. Buras and J.-M. Gérard, Upper bounds on $\epsilon^{\prime} / \epsilon$ parameters $B_{6}^{(1 / 2)}$ and $B_{8}^{(3 / 2)}$ from large-N QCD and other news, JHEP 12 (2015) 008 [arXiv:1507.06326] [INSPIRE].

[179] T. Kitahara, U. Nierste and P. Tremper, Singularity-free next-to-leading order $\Delta S=1$ renormalization group evolution and $\epsilon_{K}^{\prime} / \epsilon_{K}$ in the Standard Model and beyond, JHEP 12 (2016) 078 [arXiv: 1607.06727] [INSPIRE].

[180] A.J. Buras and J.-M. Gerard, Final state interactions in $K \rightarrow \pi \pi$ decays: $\Delta I=1 / 2$ rule vs. $\epsilon^{\prime} / \epsilon$, Eur. Phys. J. C 77 (2017) 10 [arXiv:1603.05686] [InSPIRE].

[181] Y.T. Chien, V. Cirigliano, W. Dekens, J. de Vries and E. Mereghetti, Direct and indirect constraints on CP-violating Higgs-quark and Higgs-gluon interactions, JHEP 02 (2016) 011 [arXiv: 1510.00725] [INSPIRE].

[182] Y. Jiang and M. Trott, On the non-minimal character of the SMEFT, arXiv:1612.02040 [INSPIRE].

[183] V. Cirigliano, J. Jenkins and M. Gonzalez-Alonso, Semileptonic decays of light quarks beyond the Standard Model, Nucl. Phys. B 830 (2010) 95 [arXiv: 0908.1754] [inSPIRE].

[184] V. Cirigliano, M. González-Alonso and M.L. Graesser, Non-standard charged current interactions: beta decays versus the LHC, JHEP 02 (2013) 046 [arXiv:1210.4553] [INSPIRE].

[185] E. Berkowitz et al., An accurate calculation of the nucleon axial charge with lattice QCD, arXiv: 1704.01114 [INSPIRE].

[186] V. Cirigliano, S. Gardner and B. Holstein, Beta decays and non-standard interactions in the LHC era, Prog. Part. Nucl. Phys. 71 (2013) 93 [arXiv:1303.6953] [InSPIRE]. 
[187] M. González-Alonso and J. Martin Camalich, Global effective-field-theory analysis of new-physics effects in (semi)leptonic kaon decays, JHEP 12 (2016) 052 [arXiv: 1605.07114] [INSPIRE].

[188] T. Bhattacharya et al., Probing novel scalar and tensor interactions from (ultra)cold neutrons to the LHC, Phys. Rev. D 85 (2012) 054512 [arXiv:1110.6448] [INSPIRE].

[189] O. Naviliat-Cuncic and M. González-Alonso, Prospects for precision measurements in nuclear $\beta$ decay at the LHC era, Annalen Phys. 525 (2013) 600 [arXiv:1304.1759] [INSPIRE].

[190] V. Bernard, M. Oertel, E. Passemar and J. Stern, Tests of non-standard electroweak couplings of right-handed quarks, JHEP 01 (2008) 015 [arXiv:0707.4194] [INSPIRE].

[191] W.J. Stirling and E. Vryonidou, Electroweak gauge boson polarisation at the LHC, JHEP 07 (2012) 124 [arXiv: 1204.6427] [INSPIRE].

[192] B. Grzadkowski and M. Misiak, Anomalous Wtb coupling effects in the weak radiative B-meson decay, Phys. Rev. D 78 (2008) 077501 [Erratum ibid. D 84 (2011) 059903] [arXiv: 0802.1413] [INSPIRE].

[193] J.A. Aguilar-Saavedra, A minimal set of top anomalous couplings, Nucl. Phys. B 812 (2009) 181 [arXiv: 0811.3842] [INSPIRE].

[194] G.A. Gonzalez-Sprinberg, R. Martinez and J. Vidal, Top quark tensor couplings, JHEP 07 (2011) 094 [Erratum ibid. 05 (2013) 117] [arXiv:1105.5601] [INSPIRE].

[195] J. Drobnak, S. Fajfer and J.F. Kamenik, Probing anomalous tWb interactions with rare $B$ decays, Nucl. Phys. B 855 (2012) 82 [arXiv:1109.2357] [InSPIRE].

[196] Q.-H. Cao, B. Yan, J.-H. Yu and C. Zhang, A general analysis of Wtb anomalous couplings, arXiv: 1504.03785 [INSPIRE].

[197] Z. Hioki and K. Ohkuma, Full analysis of general non-standard tbW couplings, Phys. Lett. B 752 (2016) 128 [arXiv:1511.03437] [INSPIRE].

[198] M. Schulze and Y. Soreq, Pinning down electroweak dipole operators of the top quark, Eur. Phys. J. C 76 (2016) 466 [arXiv:1603.08911] [INSPIRE].

[199] J.F. Kamenik, M. Papucci and A. Weiler, Constraining the dipole moments of the top quark, Phys. Rev. D 85 (2012) 071501 [arXiv:1107.3143] [InSPIRE].

[200] C. Zhang, N. Greiner and S. Willenbrock, Constraints on non-standard top quark couplings, Phys. Rev. D 86 (2012) 014024 [arXiv: 1201.6670] [inSPIRE].

[201] J. de Blas, M. Chala and J. Santiago, Renormalization group constraints on new top interactions from electroweak precision data, JHEP 09 (2015) 189 [arXiv:1507.00757] [INSPIRE].

[202] A. Buckley et al., Global fit of top quark effective theory to data, Phys. Rev. D 92 (2015) 091501 [arXiv: 1506.08845] [INSPIRE].

[203] A. Buckley et al., Constraining top quark effective theory in the LHC run II era, JHEP 04 (2016) 015 [arXiv: 1512.03360] [inSPIRE].

[204] O. Bessidskaia Bylund, F. Maltoni, I. Tsinikos, E. Vryonidou and C. Zhang, Probing top quark neutral couplings in the Standard Model effective field theory at NLO in QCD, JHEP 05 (2016) 052 [arXiv: 1601.08193] [INSPIRE]. 
[205] N. Castro, J. Erdmann, C. Grunwald, K. Kröninger and N.-A. Rosien, EFTfitter - a tool for interpreting measurements in the context of effective field theories, Eur. Phys. J. C 76 (2016) 432 [arXiv: 1605.05585] [INSPIRE].

[206] J. Aebischer, A. Crivellin, M. Fael and C. Greub, Matching of gauge invariant dimension-six operators for $b \rightarrow s$ and $b \rightarrow c$ transitions, JHEP 05 (2016) 037 [arXiv: 1512.02830] [INSPIRE].

[207] V. Cirigliano, W. Dekens, J. de Vries and E. Mereghetti, Constraining the top-Higgs sector of the Standard Model effective field theory, Phys. Rev. D 94 (2016) 034031 [arXiv: 1605.04311] [INSPIRE].

[208] V. Cirigliano, W. Dekens, J. de Vries and E. Mereghetti, Is there room for CP-violation in the top-Higgs sector?, Phys. Rev. D 94 (2016) 016002 [arXiv: 1603.03049] [InSPIRE].

[209] A. Crivellin, Effects of right-handed charged currents on the determinations of $\left|V_{u b}\right|$ and $\left|V_{c b}\right|$, Phys. Rev. D 81 (2010) 031301 [arXiv:0907.2461] [INSPIRE].

[210] A.J. Buras, K. Gemmler and G. Isidori, Quark flavour mixing with right-handed currents: an effective theory approach, Nucl. Phys. B 843 (2011) 107 [arXiv:1007.1993] [InSPIRE].

[211] M. Beneke, G. Buchalla, M. Neubert and C.T. Sachrajda, QCD factorization for exclusive, nonleptonic B meson decays: general arguments and the case of heavy light final states, Nucl. Phys. B 591 (2000) 313 [hep-ph/0006124] [INSPIRE].

[212] J. Chay and C. Kim, Analysis of the QCD improved factorization in $B \rightarrow J / \psi K$, hep-ph/0009244 [INSPIRE].

[213] M. Beneke, Soft-collinear factorization in B decays, Nucl. Part. Phys. Proc. 261-262 (2015) 311 [arXiv: 1501.07374] [InSPIRE].

[214] A.J. Buras, S. Jager and J. Urban, Master formulae for $\Delta F=2 N L O Q C D$ factors in the Standard Model and beyond, Nucl. Phys. B 605 (2001) 600 [hep-ph/0102316] [InSPIRE].

[215] X.-G. He, J. Tandean and G. Valencia, Penguin and box diagrams in unitary gauge, Eur. Phys. J. C 64 (2009) 681 [arXiv:0909.3638] [InSPIRE].

[216] ETM collaboration, N. Carrasco et al., B-physics from $N_{f}=2$ tmQCD: the Standard Model and beyond, JHEP 03 (2014) 016 [arXiv: 1308.1851] [INSPIRE].

[217] A.J. Buras and J. Girrbach, Towards the identification of new physics through quark flavour violating processes, Rept. Prog. Phys. 77 (2014) 086201 [arXiv: 1306.3775] [INSPIRE]. 